\title{
Synthesis of Imidazolidin-2-ones and Imidazol-2-ones via Base- Catalyzed Intramolecular Hydroamidation of Propargylic Ureas under Ambient Conditions
}

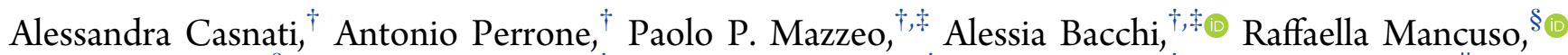 \\ Bartolo Gabriele, ${ }^{\S ®}$ Raimondo Maggi, ${ }^{\dagger}$ Giovanni Maestri, ${ }^{\dagger}{ }^{\dagger}$ Elena Motti, ${ }^{\dagger}$ András Stirling, ${ }^{*}, \|_{\odot}$ \\ and Nicola Della Ca'*,† \\ ${ }^{\dagger}$ Department of Chemistry, Life Sciences and Environmental Sustainability, University of Parma, 43124 Parma, Italy \\ ${ }^{\ddagger}$ Biopharmanet-tec, Parco delle Scienze, 27/A, 43124 Parma, Italy \\ ${ }^{\S}$ Dipartimento di Chimica e Tecnologie Chimiche, Università della Calabria, 87036 Arcavacata di Rende, Cosenza, Italy \\ "Theoretical Chemistry Research Group, Institute of Organic Chemistry, Research Centre for Natural Sciences, Budapest, Hungary
}

Supporting Information

ABSTRACT: The first organo-catalyzed synthesis of imidazolidin-2-ones and imidazol-2-ones via intramolecular hydroamidation of propargylic ureas is reported. The phosphazene base BEMP turned out to be the most active organo-catalyst compared with guanidine and amidine bases. Excellent chemoand regioselectivities to five-membered cyclic ureas have been achieved under ambient conditions, with a wide substrate scope and exceptionally short reaction times (down to $1 \mathrm{~min}$ ).

A base-mediated isomerization step to an allenamide intermediate is the most feasible reaction pathway to give imidazol-2-ones, as suggested by DFT studies.

\section{INTRODUCTION}

Imidazol-2-ones and their analogues are omnipresent structural motifs of bioactive pharmaceutical compounds and natural alkaloids (Figure 1). ${ }^{1}$ Five-membered cyclic ureas are also flexible key intermediates in synthetic transformations ${ }^{2}$ and useful chiral auxiliaries in enantioselective syntheses. ${ }^{3}$

The high value of these heterocycles for synthetic and pharmaceutical chemistry has driven continuous efforts in the development of sustainable and more efficient protocols. Traditional synthetic routes to imidazolidin-2-ones are mainly
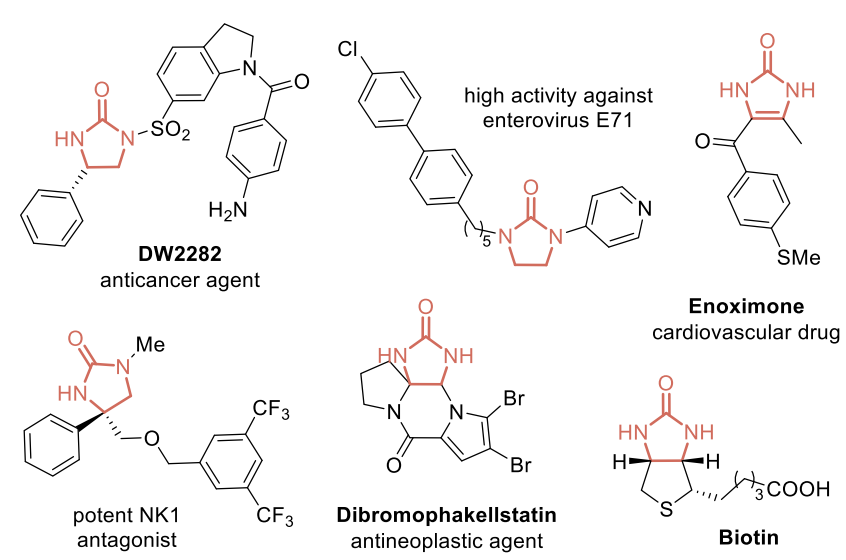

Figure 1. Selected bioactive 2-imidazol(idin)one derivatives.

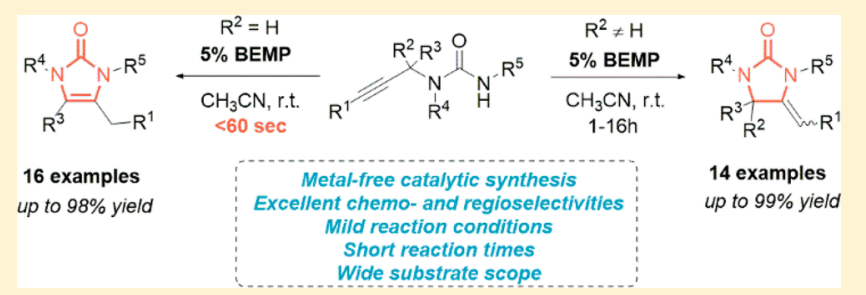

based on the carbonylation of diamines, ${ }^{4}$ which can require toxic carbonylating agents, high-pressure facilities, and harsh reaction conditions. Metal-catalyzed diamination of olefins ${ }^{5}$ and aziridine ring expansion ${ }^{6}$ are elegant strategies; however, the widespread application suffers from their inherent sophistication. The intramolecular hydroamidation approach ${ }^{7}$ offers a powerful, atom- and step-economical alternative route to easily access richly decorated five-membered cyclic ureas. $^{4 a, 8,9}$ In this context, propargylureas have been extensively investigated as readily available precursors for the synthesis of both imidazolin-2-ones and imidazolidin-2-ones. The control of chemo- ( $\mathrm{O}$ vs $\mathrm{N}$ cyclization) and regio- (exo- vs endo-) selectivity in the cyclization step is generally addressed by means of transition metal catalysts. ${ }^{8 a-h, 8,10}$ As a relevant example, a $\mathrm{Ag}(\mathrm{I})$-catalyzed one-pot protocol for the synthesis of 2-imidazolones through cycloisomerization of an in situ formed propargylic urea was disclosed by Van der Eycken in 2011. ${ }^{8 \mathrm{~d}}$ More recently, Reddy and co-workers have reported the synthesis of indole-fused cyclic urea derivatives through a Ag-catalyzed sequence that involves an elegant double intramolecular hydroamidation process. $^{8 \mathrm{~b}}$ These interesting methods require however a high catalyst loading (up to $30 \mathrm{~mol}$ $\%)$ and harsh reaction conditions. More sustainable metal-free protocols to efficiently access imidazol-2-ones and imidazoli-

Received: January 8, 2019

Published: February 21, 2019 
din-2-ones from propargylic ureas have been also developed by using a stoichiometric amount of bases or salts. ${ }^{9}$ For instance, Dethe and Lubell have recently proposed the use of $\mathrm{NaOH}^{9 \mathrm{a}}$ and $\mathrm{NaH}^{9 \mathrm{~b}}$ for the synthesis of imidazole-2-thione and $\mathrm{N}$ amino-imidazolin-2-ones, respectively, under mild conditions. Despite the usefulness of these methods, a base-catalyzed intramolecular hydroamidation approach to imidazol-2-one derivatives is still in high demand and, to date, totally unprecedented.

Herein, we disclose the first base-catalyzed intramolecular hydroamidation of propargylic ureas to highly substituted imidazolidin-2-ones and imidazol-2-ones. Notable features of our methodology include (i) excellent chemo- and regioselectivity to five-membered cyclic ureas, (ii) a wide substrate scope and high functional group tolerance, (iii) very mild reaction conditions, and (iv) remarkably short reaction times. Further, thanks to key mechanistic insights on the reaction pathway, we demonstrated the feasibility of a one-pot stepeconomical protocol starting from propargyl amines and isocyanates.

\section{RESULTS AND DISCUSSION}

Inspired by our recent studies on the base-catalyzed synthesis of imidazolidin-2-ones from propargylamines, $\mathrm{CO}_{2}$, and primary amines, ${ }^{11}$ we started to investigate the intramolecular hydroamidation reaction of propargylic ureas. ${ }^{12}$ Guanidine base TBD, widely exploited in a plethora of catalytic transformations, ${ }^{13}$ including the above-mentioned synthesis of imidazolidin-2-ones, ${ }^{11}$ was initially employed as a catalyst for the hydroamidation of propargylic urea 1a (Table 1) using similar reaction conditions. We found that propargylic urea 1a in the presence of $10 \%$ of $\mathrm{TBD}$ at $100{ }^{\circ} \mathrm{C}$ in anhydrous acetonitrile converted quantitatively to imidazolidinone $\mathbf{2 a}$ (Table 1, entry 1). Excellent results were also achieved at lower temperatures (Table 1, entries 2 and 3), and therefore, subsequent experiments were tested at $\mathrm{rt}\left(22-23^{\circ} \mathrm{C}\right)$. A series of similar organic bases were then evaluated (Table 1 , entries 4-7). MTBD and BTMG were found to be less effective, leading to imidazolidinone $\mathbf{2 a}$ in $82 \%$ and $67 \%$ yield, respectively (Table 1 , entries 4 and 5). On the contrary, DBU and TMG turned out to be totally inactive in promoting the desired cyclization (Table 1, entries 6 and 7). Notably, the catalytic activity seemed to follow the strength of the bases. We then resorted to 2-tert-butylimino-2-diethylamino-1,3-dimethylperhydro-1,3,2-diazaphosphorine (BEMP), which is a stronger base that proved to be useful in other hydroamination reactions. ${ }^{14}$ Gratifyingly, the use of $10 \mathrm{~mol} \%$ of BEMP afforded $2 \mathrm{a}$ in quantitative yield (Table 1, entry 8). Furthermore, the reaction time decreased dramatically to 30 min. This significant improvement allowed us to decrease the amount of catalyst down to $1 \mathrm{~mol} \%$, while still maintaining high reaction performance (Table 1 , entries 9 and 10). Other solvents were then tested (Table 1 , entries 11-13), and we found that $\mathrm{MeCN}$ was crucial to trigger an efficient reaction. Notably, the presence of water in the mixture, detrimental for other protocols, ${ }^{8 \mathrm{~d}, 9 \mathrm{~b}}$ did not affect the final outcome (Table 1, entry 14). Finally, no reaction took place in the control experiment in the absence of catalyst (Table 1, entry 15).

To illustrate the scope of this methodology, a series of propargyl ureas bearing a quaternary carbon $\alpha$ to the triple bond were then prepared. Under optimal conditions (Table 1, entry 9), propargylic ureas $1 \mathbf{a}-\mathbf{1}$ reacted smoothly to give the corresponding imidazolidin-2-ones $\mathbf{2 a - 1}$ in excellent yields
Table 1. Optimization Study ${ }^{a}$

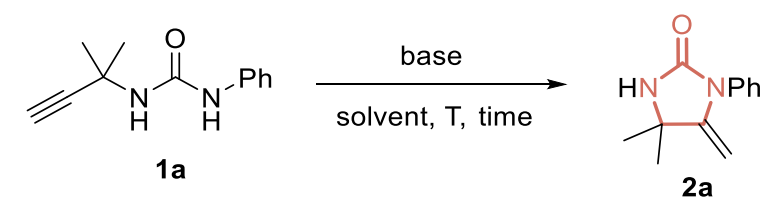

$\begin{array}{cllccc}\text { entry } & \text { base (mol \%) } & \text { solvent } & \text { T }\left({ }^{\circ} \mathrm{C}\right) & \text { time }(\mathrm{h}) & \text { yield }(\%)^{b} \text { 2a } \\ 1 & \text { TBD (10) } & \mathrm{MeCN} & 100 & 24 & 99 \\ 2 & \text { TBD (10) } & \mathrm{MeCN} & 50 & 24 & 99 \\ 3 & \text { TBD (10) } & \mathrm{MeCN} & \mathrm{rt} & 24 & 99 \\ 4 & \text { MTBD (10) } & \mathrm{MeCN} & \mathrm{rt} & 24 & 82 \\ 5 & \text { BTMG (10) } & \mathrm{MeCN} & \mathrm{rt} & 24 & 67 \\ 6 & \text { DBU (10) } & \mathrm{MeCN} & \mathrm{rt} & 24 & - \\ 7 & \text { TMG (10) } & \mathrm{MeCN} & \mathrm{rt} & 24 & - \\ 8 & \text { BEMP (10) } & \mathrm{MeCN} & \mathrm{rt} & 0.5 & 99 \\ 9 & \text { BEMP (5) } & \mathrm{MeCN} & \mathrm{rt} & 1 & 99 \\ 10 & \text { BEMP (1) } & \mathrm{MeCN} & \mathrm{rt} & 7 & 93 \\ 11 & \text { BEMP (5) } & \mathrm{EtOAc} & \mathrm{rt} & 24 & - \\ 12 & \text { BEMP (5) } & \mathrm{THF} & \mathrm{rt} & 24 & - \\ 13 & \text { BEMP (5) } & \mathrm{MeOH} & \mathrm{rt} & 24 & - \\ 14 & \text { BEMP (5) } & \mathrm{MeCN} & \mathrm{rt} & 1 & 99 \\ 15 & - & \mathrm{MeCN} & \mathrm{rt} & 24 & -\end{array}$

${ }^{a}$ Reaction conditions: $1 \mathrm{a}(0.4 \mathrm{mmol})$, base $(1-10 \mathrm{~mol} \%)$, solvent (4 $\mathrm{mL}$ ). ${ }^{b}$ Yields of 2 a were determined via ${ }^{1} \mathrm{H}$ NMR analysis using methylbenzoate as internal standard. ${ }^{c} 0.4 \mathrm{mmol}$ (1 equiv) of $\mathrm{H}_{2} \mathrm{O}$ was added. ${ }^{d} K_{\mathrm{a}}$ is referred to its conjugate acid in MeCN. ${ }^{13}{ }^{e}$ Not determined. $^{14}$
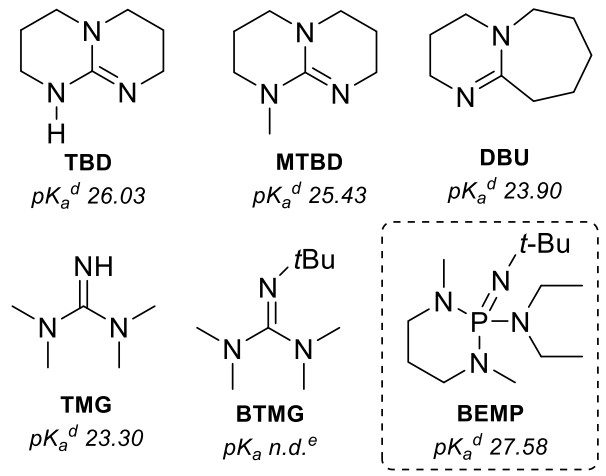

(Table 2). Reactions reached completion within 1 to $16 \mathrm{~h}$ at rt. Both electron-donating $(\mathrm{Me})$ and electron-withdrawing ( $\mathrm{F}$, $\mathrm{CO}_{2} \mathrm{Me}$ ) groups on the aryl substituent were well tolerated in this simple and robust methodology $(\mathbf{2} \mathbf{b}-\mathbf{d}, 82-98 \%)$. The reaction rate is slower increasing the hindrance on the carbon $\alpha$ to the triple bond ( $2 \mathrm{e}$ and $\mathbf{2 f}, 3$ and $16 \mathrm{~h}$ ). However, the presence of a substituent on the nitrogen $\beta$ to the triple bond caused a lower yield of the corresponding cyclic urea, as shown in the case of $2 \mathrm{~g}\left(\mathrm{R}^{3}=\mathrm{Bn}, 52 \%\right)$. Propargylic ureas bearing an internal triple bond led to the corresponding imidazolidin-2ones in high yield $(\mathbf{2} \mathbf{h}-\mathbf{1}, \mathbf{8 3}-\mathbf{9 7 \%})$. Aryl and alkyl groups on the triple bond were well tolerated. In all cases, the $Z$ product was the preferred one, ${ }^{15}$ although the stereocontrol was not complete. Ureas bearing an alkyl substituent in $\mathrm{R}^{4}$, such as $n$ $\mathrm{Bu}$, were unreactive under these optimized conditions. Interestingly, urea $1 \mathbf{m}$ that bears an ester group in a suitable position $\left(\mathrm{R}^{4}=\mathrm{CH}_{2} \mathrm{CO}_{2} \mathrm{Et}\right)$ led to hydantoin $2 \mathbf{m}^{\prime}(72 \%)$, ${ }^{9 \mathrm{c}}$ leaving the triple bond untouched.

We also tested the reactivity of a propargylic thiourea, which is reported to give imidazole-2-thiones under basic conditions. $^{9 a}$ On the contrary, thiazolidin-2-imine $2 \mathbf{n}^{\prime}$ was 
Table 2. Scope of the BEMP-Catalyzed Synthesis of Imidazolidin-2-ones $2^{a, b}$
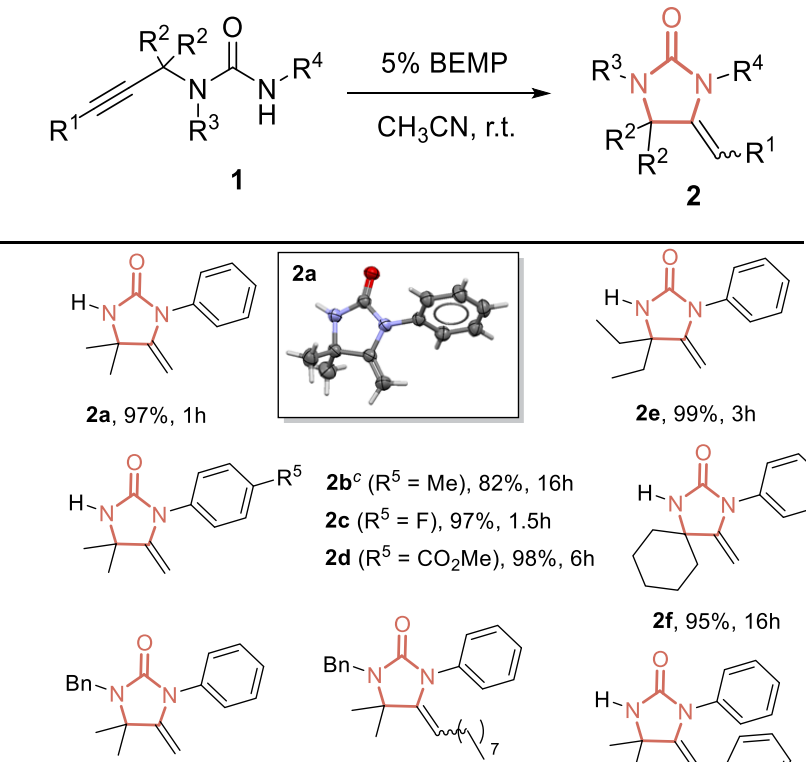

2e, $99 \%, 3 \mathrm{~h}$

$2 g, 52 \%, 16 \mathrm{~h}$
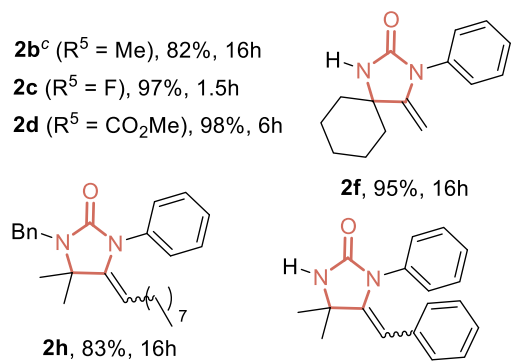

$$
\begin{aligned}
& \text { 2h, } 83 \%, 16 h \\
& \text { ZIE } 1: 0.06
\end{aligned}
$$
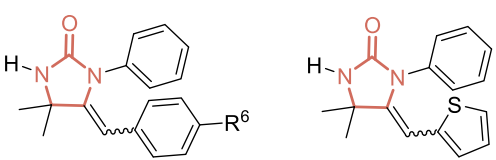

2j $\left(R^{6}=C_{3}\right), 93 \%, 4 h, Z / E 1: 0.3$

2I, $97 \%, 1.5 \mathrm{~h}$

2k $\left(\mathrm{R}^{6}=\mathrm{OMe}\right), 84 \%, 16 \mathrm{~h}, \mathrm{Z} / \mathrm{E} 1: 0.04$

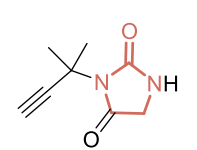

$2 \mathrm{~m}^{\prime}, 72 \%, 50^{\circ} \mathrm{C}, 16 \mathrm{~h}$
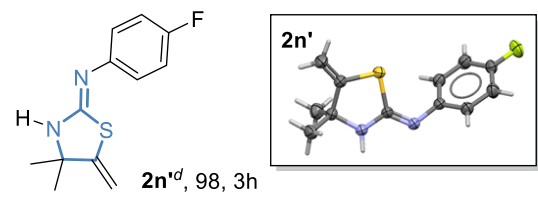

2i, $87 \%, 16 \mathrm{~h}$, Z/E 1:0.25

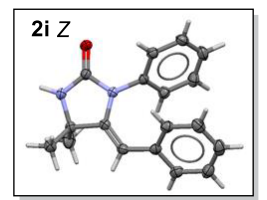

${ }^{a}$ Reaction conditions: 1 ( $\left.0.4 \mathrm{mmol}\right)$, BEMP ( $\left.5 \mathrm{~mol} \%\right), \mathrm{MeCN}$ (4 $\mathrm{mL})$, room temperature. ${ }^{b}$ Yields of $2 .{ }^{c}$ The reaction was performed at $40{ }^{\circ} \mathrm{C}$. ${ }^{d}$ Compound $2 n^{\prime}$ was obtained directly from propargylic amine and isothiocyanate without catalyst (see SI for details).

selectively obtained directly from propargylic amine and isothiocyanate precursors through intramolecular S-cyclization without a catalyst. The structure of $\mathbf{2} \mathbf{n}^{\prime}$ was undoubtedly confirmed by SC-XRD (see the Supporting Information (SI) for details). ${ }^{15}$

We then further explored the substrate scope of the transformation by employing a variety of propargylic ureas 3 bearing a tertiary carbon $\alpha$ to the triple bond (Table 3 ). In this case, selective formation of imidazol-2-ones 4 was observed, with a formal double bond shift from the exo- to the endocyclic position. To our delight, reaction times were impressively shortened. Upon reagent mixing, the reaction completes in about $1 \mathrm{~min}$. The protocol was again characterized by a remarkable tolerance of valuable functional groups, including $\mathrm{Cl}(4 \mathbf{i}, 86 \%), \mathrm{Br}(4 \mathbf{k}, 68 \%), \mathrm{F}$ (4f, 93\%), OMe (4h, $77 \%)$, or vinyl $\left(4 \mathbf{j}^{15}{ }^{15} 75 \%\right)$ substituents. It is worth noting that this ample tolerance ensures vast handles for further functionalizations. Alkyl substituents on the triple bond $\left(\mathrm{R}^{1}\right)$ or on terminal nitrogen $\left(R^{4}\right)$ gave low yields, as shown for example for $4 b$ and
Table 3. Scope of the BEMP-Catalyzed Synthesis of Imidazol-2-ones $4^{a, b}$

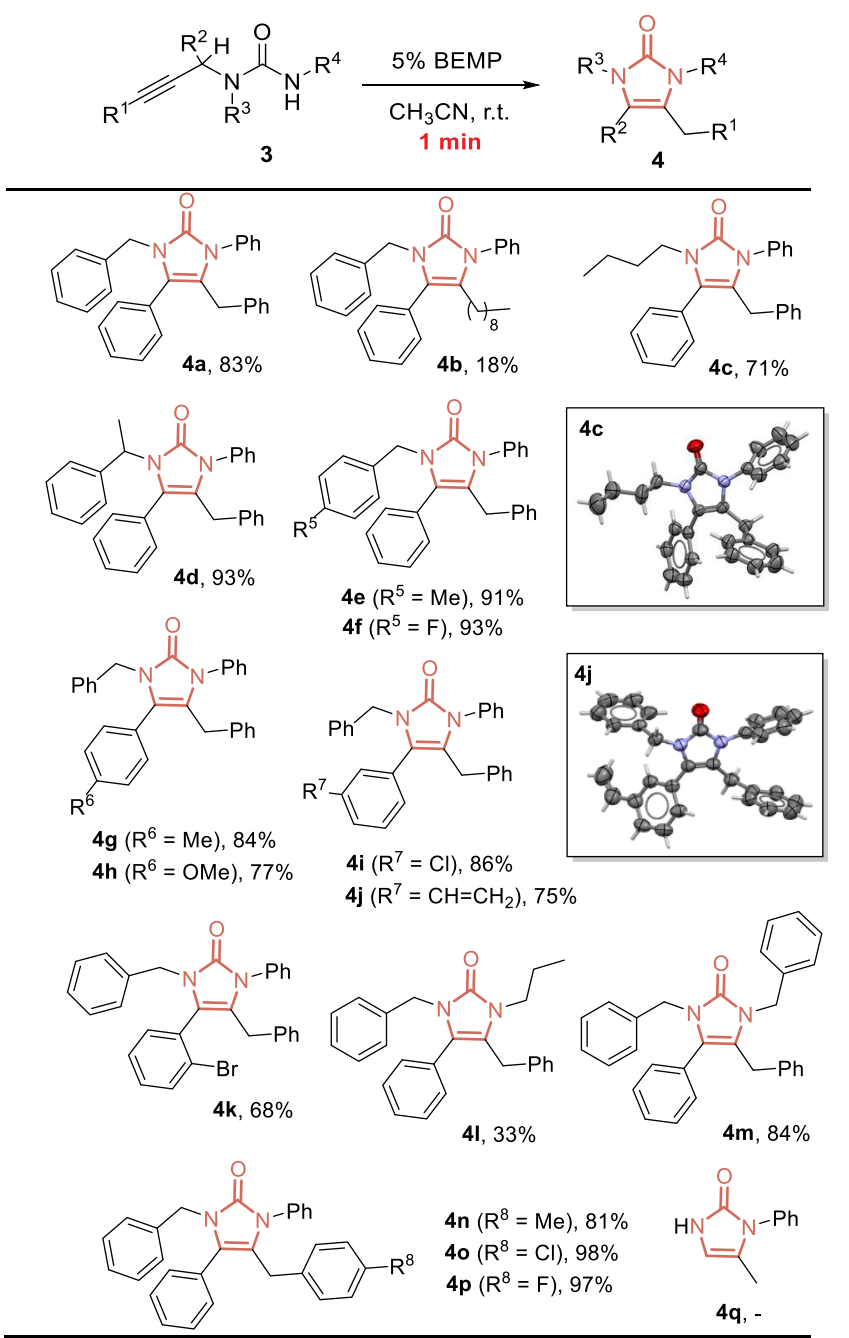

${ }^{a}$ Reaction conditions: 3 (0.4 mmol), BEMP ( $\left.5 \mathrm{~mol} \%\right), \mathrm{MeCN}$ (4 $\mathrm{mL})$, room temperature, $1 \mathrm{~min} .{ }^{b}$ Yields of 4 .

41 ( $18 \%$ and $33 \%$ yield, respectively). Alkyl or benzyl substituents on nitrogen $\beta$ to the triple bond $\left(\mathrm{R}^{3}\right)$ led to an excellent yield of product 4 , even for more sterically demanding groups, as in the case of $\mathbf{4 d}$ (93\% yield). This is complementary to the trend observed with products $2(2 \mathrm{~g}$, Table 2). Electronic effects exerted by substituents in the para position on the aromatic ring $\left(\mathrm{R}_{1}\right)$ were also explored. Urea $3 \mathbf{n}$ bearing a tolyl group on the triple bond $\left(\mathrm{R}^{1}=4-\mathrm{MeC}_{6} \mathrm{H}_{4}\right)$ led to the corresponding imidazolone $4 \mathbf{n}$ in $81 \%$ yield, which is slightly less than those observed for $4 a\left(\mathrm{R}^{1}=\mathrm{C}_{6} \mathrm{H}_{5}\right)$. The best results were obtained with electron-withdrawing groups, such as $\mathrm{Cl}(4 \mathbf{o}, 98 \%)$ and $\mathrm{F}(4 \mathrm{p}, 97 \%)$. Finally, the unsubstituted propargylic urea $3 \mathrm{q}\left(\mathrm{R}^{1}, \mathrm{R}^{2}, \mathrm{R}^{3}=\mathrm{H}\right)$ did not provide the corresponding imidazolone $\mathbf{4 q}$, being unreactive under these conditions.

To further demonstrate the applicability of this protocol, the one-pot synthesis of imidazol-2-one derivatives starting from propargylic amines and isocyanates was performed under standard conditions. Gratifyingly, compound 2a was quantitatively obtained in $1 \mathrm{~h}$ from 2-methylbut-3-yn-2-amine and phenyl isocyanate (Scheme 1a). Without further optimization, imidazolinone 4a was synthesized directly from the propargyl- 
Scheme 1. Representative Examples of the One-Pot Synthesis of Imidazolidin-2-one 2a and Imidazolin-2-one 4a from Propargylic Amines and Isocyanates a)<smiles>C#CC(C)(C)N</smiles>
$1 \mathrm{~h}$

b)

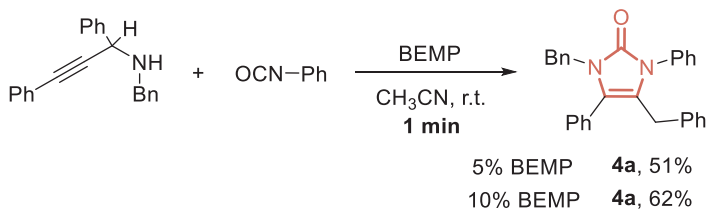

amine $3 a$ and phenyl isocyanate in $62 \%$ yield, using $10 \mathrm{~mol} \%$ of BEMP (Scheme 1b). Noteworthy, this intermolecular reaction was still complete within $1 \mathrm{~min}$.

Intrigued by the complete chemoselectivity and site selectivity features as well as by the unexpectedly fast formation of imidazol-2-ones $\mathbf{4}$ under very mild conditions, we carried out computational studies to gain insights into the reaction mechanism. Investigation began by employing the Gaussian09 package $^{16}$ using the range separated, hybrid $\omega \mathrm{b} 97 \mathrm{xd}$ functional. ${ }^{17}$ The possible reaction routes have been studied in terms of solvent-corrected Gibbs free energy values (see SI for details). Figure 2 shows the most feasible

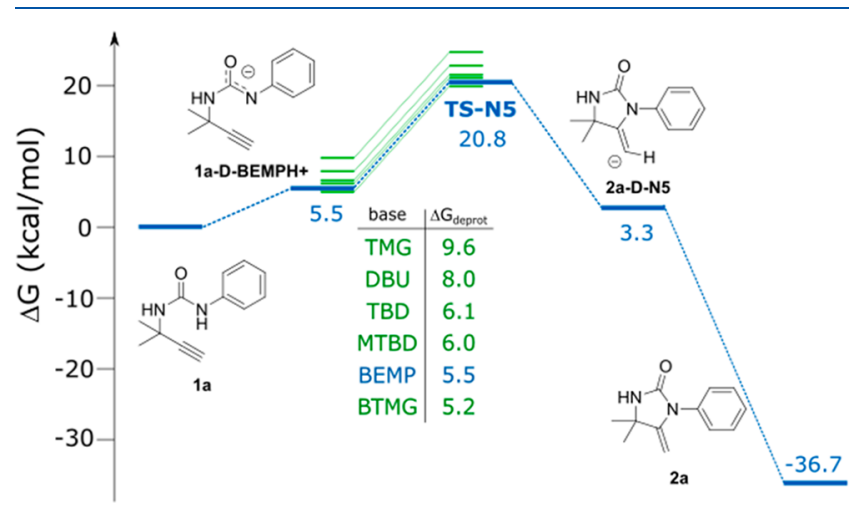

Figure 2. Gibbs free energy profile of the formation of imidazolidin-2one 2a (N-5-exo-dig cyclization pathway). Color code used for the effect of bases: (blue) BEMP as base; (green, from top to bottom) TMG, DBU, TBD, MTBD, BTMG as base. ${ }^{21}$

mechanism to generate imidazolidin-2-one 2a from propargylic urea 1a. The reaction starts with the abstraction of the most acidic urea proton by the base, which is a slightly endergonic process. We obtained good correlation between the $\mathrm{p} K_{\mathrm{a}}$ of the bases and the energonicity of the deprotonation step. ${ }^{18}$ The levels obtained with the strongest base (BEMP) are shown in blue. The deprotonation yields 1a-D-BEMPH+ $(\Delta G+5.5$ $\mathrm{kcal} / \mathrm{mol}$ ). We observed that, upon this proton transfer, the conjugated acid/base pair dissociates as higher $\Delta G$ values were obtained modeling a tight ion pair. We explained this by the entropy penalty of the association of the two ions, ${ }^{19}$ and also by the enhanced solvation of the two independent ions which together boost therefore dissociation in the polar $\mathrm{MeCN}$ solvent. The deprotonated urea can then react with the triple bond in four different ways depending on the cyclization fashion. $\mathrm{N}$ - or $\mathrm{O}$-carbon bond formation occurs via either 5exo- or 6-endo-dig cyclization modes. The formation of the anionic form of product 2a (2a-D-N5) is the most favorable pathway both kinetically and thermodynamically (not shown here; see SI, Figure S1). Eventually, the cyclization is followed by the strongly exergonic protonation yielding the product 2 a $(\Delta G-36.7 \mathrm{kcal} / \mathrm{mol})$. The rate-determining step is the cyclization, which requires a moderate $20.8 \mathrm{kcal} / \mathrm{mol}$ activation free energy (from 1a to TS-N5) in the presence of BEMP. This is fully compatible with the observed reaction rate. ${ }^{20}$ The calculated trend among different bases evenly correlates with the experimental results listed in Table 1. Indeed, stronger bases proved to be better catalysts, as they can more easily deprotonate the substrate (Figure 2).

Second, formation of imidazol-2-one 4a from 3a was investigated. The initial base-catalyzed isomerization of the triple bond to the corresponding allenamide was the most favored pathway to imidazol-2-ones 4a (Figure 3, blue

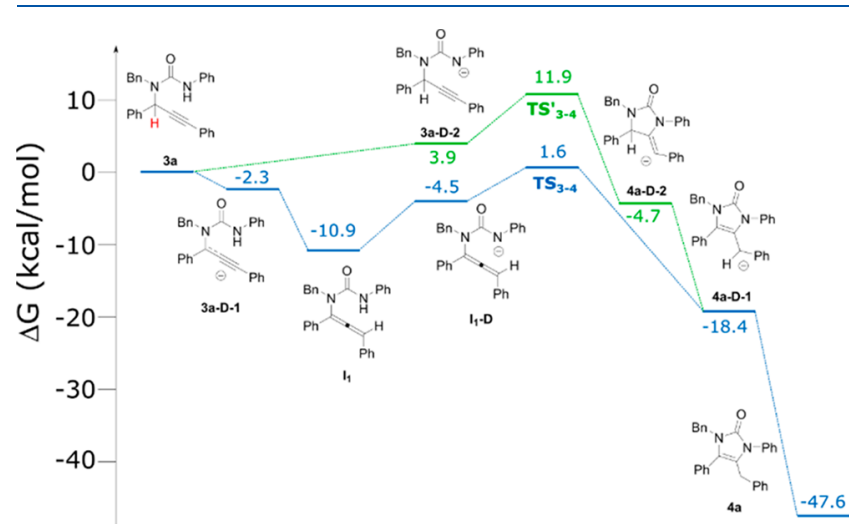

Figure 3. Gibbs free energy profile of the formation of imidazol-2-one 4a, employing BEMP as catalyst. Blue profile: allene-route. Green profile: mechanism analogue to the one presented in Figure 2.

pathway). The proton of $\mathbf{3 a}$ highlighted in red became the most acidic one. This ensures that the initial allenamide formation is preferred to urea deprotonation (Figure 3, green pathway). Hence, the resting state under the reaction conditions is allenamide $I_{1}$. Isomerization of the propargylic arm to the corresponding allenamide is highly plausible under strongly basic conditions. ${ }^{22}$ Then, the $\mathrm{N}-\mathrm{H}$ deprotonation can occur and leads to the formation of the imidazol-2-one ring (4a-D-1) through a low barrier transition state. The final protonation yields the neutral product in a highly exergonic step. The calculations confirmed that the central carbon of the allene moiety is the most electron-deficient, ${ }^{23}$ which explains the preference for the five-membered ring formation. The barrier for the cyclization step is only $12.5 \mathrm{kcal} / \mathrm{mol}$ (from $\mathrm{I}_{1}$ to $\left.\mathrm{TS}_{3-4}\right)$. This suggests a very fast reaction, in perfect agreement with the experimental observations. ${ }^{24}$

\section{CONCLUSION}

In summary, we have developed the first organo-catalyzed method to access imidazolidin-2-ones and imidazol-2-ones from propargylureas under ambient conditions. The protocol is simple, tolerates the presence of water, and provides fivemembered cyclic ureas with excellent space-time yields. DFT calculations provided support for the nonassisted cyclization of deprotonated urea in the imidazolidin-2-one formation and revealed the involvement of an allene intermediate in the imidazol-2-one pathway. The one-pot protocol starting from propargylic amines and isocyanates was also demonstrated. We 
anticipate that the unique features of this method will ensure wide interest and vast application within the chemistry community at large.

\section{EXPERIMENTAL SECTION}

General Methods. All chemicals were purchased from commercial sources and used as received, unless otherwise noted. Solvents were dried and stored over molecular sieves previously activated in an oven $\left(450{ }^{\circ} \mathrm{C}\right.$ overnight $)$. Organocatalyzed reactions were carried out under nitrogen using standard Schlenk techniques. Reactions were analyzed by thin layer chromatography (TLC) on silica gel 60 F254. GC analyses were performed with an Agilent Tenchnologies 7820A equipped with an FID detector and a $30 \mathrm{~m}$ capillary column. GC-MS analyses $(\mathrm{m} / z$, relative intensity\%) were performed with an Agilent Technologies $6890 \mathrm{~N}$ gas chromatograph coupled to a $5973 \mathrm{~N}$ mass selective detector (Agilent Technologies) working at $70 \mathrm{eV}$ ionizing voltage. Column chromatography was performed on silica gel 60 (70-230 mesh). ${ }^{1} \mathrm{H},{ }^{13} \mathrm{C}$, and ${ }^{19} \mathrm{~F}$ NMR spectra were recorded at 300 $\mathrm{K}$ on a Bruker Avance 400 or $300 \mathrm{MHz}$ using the solvent as the internal standard (7.26 ppm for ${ }^{1} \mathrm{H}$ NMR and $77.00 \mathrm{ppm}$ for ${ }^{13} \mathrm{C}$ $\mathrm{NMR}$ for $\mathrm{CDCl}_{3}$ ). The terms $\mathrm{m}, \mathrm{s}, \mathrm{d}, \mathrm{t}, \mathrm{q}$, and quint represent multiplet, singlet, doublet, triplet, quadruplet, and quintuplet, respectively, and the term br means a broad signal. Exact masses were recorded on an LTQ ORBITRAP XL Thermo Mass Spectrometer (ESI source). Melting points were measured with an electrothermal apparatus and are uncorrected.

Experimental Procedures. Synthesis of Propargylic Ureas $1 a-$ f, $1 \mathrm{~m}, 3 \mathrm{q}^{25}$ A dry round-bottom two-necked flask, containing a magnetic stir bar, was charged with the selected isocyanate $(2.5$ $\mathrm{mmol})$, the desired propargylic amine $(2.5 \mathrm{mmol})$, and dry THF $(25$ $\mathrm{mL}$ ). The resulting mixture was stirred at room temperature overnight. The solvent was removed under reduced pressure. Yields were quantitative, and propargylic ureas $\mathbf{1 a}-\mathbf{f}, \mathbf{1 m}$, and $\mathbf{3 q}$ were employed without further purification.

Synthesis of Propargylic Ureas 1i-I through Sonogashira Coupling. ${ }^{26}$ A Schlenk-type flask equipped with a magnetic stir bar was charged with $\mathrm{Pd}\left(\mathrm{PPh}_{3}\right)_{2} \mathrm{Cl}_{2}(0.02 \mathrm{mmol}, 2 \mathrm{~mol} \%, 14 \mathrm{mg}), \mathrm{CuI}$ $(0.07 \mathrm{mmol}, 7 \mathrm{~mol} \%, 13 \mathrm{mg})$, and $\mathrm{Et}_{3} \mathrm{~N}(5 \mathrm{~mL})$ under an inert atmosphere $\left(\mathrm{N}_{2}\right)$. The aryl iodide $(1 \mathrm{mmol}, 1$ equiv) and the propargylic urea (1.2 equiv) were dissolved in dry $\mathrm{CH}_{2} \mathrm{Cl}_{2}(7 \mathrm{~mL})$ and then added. The reaction mixture was stirred at room temperature overnight. The crude mixture was filtered through a fritted funnel, the solid residue was washed with $\mathrm{CH}_{2} \mathrm{Cl}_{2}$, and the combined organic solutions were concentrated under reduced pressure. Purification via flash column chromatography yielded the desired propargyl urea.

Synthesis of Propargylic Urea 19. ${ }^{27}$ A solution of 2-methylbut-3yn-2-amine $(5 \mathrm{mmol}, 519 \mu \mathrm{L})$ and benzaldehyde $(5 \mathrm{mmol}, 507 \mu \mathrm{L})$ in $\mathrm{MeOH}(10 \mathrm{~mL})$ was stirred at room temperature overnight. Sodium borohydride $(10 \mathrm{mmol}, 378 \mathrm{mg})$ was then added to the reaction mixture at $0{ }^{\circ} \mathrm{C}$ for $1 \mathrm{~h}$ under stirring. Then the reaction crude was diluted with water $(25 \mathrm{~mL})$ and extracted with $\mathrm{CH}_{2} \mathrm{Cl}_{2}(3 \times 40 \mathrm{~mL})$. The organic layers were combined, dried over $\mathrm{MgSO}_{4}$, filtered, and concentrated under reduced pressure. The crude reaction mixture was purified by silica gel column chromatography (Eluent: hexane/ethyl acetate $7: 3)$ to afford N-benzyl-2-methylbut-3-yn-2-amine in 55\% yield $(476 \mathrm{mg})$.

A dry round-bottom two-necked flask, containing a magnetic stir bar, was charged with phenyl isocyanate $(2.5 \mathrm{mmol}, 297 \mathrm{mg}), \mathrm{N}$ benzyl-2-methylbut-3-yn-2-amine ( $2.5 \mathrm{mmol}, 432 \mathrm{mg})$, and dry THF $(25 \mathrm{~mL}) .{ }^{25}$ The resulting mixture was stirred at room temperature for $1 \mathrm{~h}$. The solvent was removed under reduced pressure. The yield is quantitative, and propargylic urea $\mathbf{1 g}$ was employed without further purification.

Synthesis of N-Benzyl-2-methyldodec-3-yn-2-amine. ${ }^{28}$ A test tube equipped with a magnetic stirrer was charged with $\mathrm{CuI}(0.6$ $\mathrm{mmol}, 30 \% \mathrm{~mol}, 114 \mathrm{mg}$ ). The test tube was sealed and flushed with $\mathrm{N}_{2}$ and then was charged with dec-1-yne $(2 \mathrm{mmol}, 361 \mu \mathrm{L})$, acetone $(2 \mathrm{mmol}, 147 \mu \mathrm{L})$, and benzylamine $(2 \mathrm{mmol}, 219 \mu \mathrm{L})$. The test tube was then placed in an oil bath at $75^{\circ} \mathrm{C}$, and it was allowed to stir overnight. The crude was purified by flash column chromatography using hexane/ethyl acetate (9:1) as eluent to give the desired amine (206 $\mathrm{mg}, 31 \%$ yield) as a transparent oil.

Synthesis of $\alpha$-Monosubstituted Propargylic Amines. ${ }^{28}$ In a typical experiment, $\mathrm{CuI}(0.6 \mathrm{mmol}, 30 \% \mathrm{~mol}, 114 \mathrm{mg})$ was charged in a test tube equipped with a magnetic stirrer. The test tube was sealed and flushed with $\mathrm{N}_{2}$ and then was charged with the alkyne $(2 \mathrm{mmol})$, the aldehyde $(2 \mathrm{mmol})$, and the amine $(2 \mathrm{mmol})$. The test tube was then placed in an oil bath at $75{ }^{\circ} \mathrm{C}$, and it was allowed to stir overnight. The crude reaction mixture was purified by silica gel column chromatography with a mixture of hexane/ethyl acetate (from 95:5 to 80:20) to provide the desired secondary propargylic amine.

Synthesis of Propargylic Ureas $1 h$ and $3 a-p .^{25}$ A dry roundbottom two-necked flask, containing a magnetic stir bar, was charged with the selected isocyanate $(1 \mathrm{mmol})$, the desired propargylic amine $(1 \mathrm{mmol})$, and dry THF $(10 \mathrm{~mL})$. The resulting mixture was stirred at room temperature for $1 \mathrm{~h}$. The solvent was removed under reduced pressure. Yields were quantitative for propargylic ureas $3 a-k, 3 n-p$, which were employed without further purification. Silica gel column chromatography (Eluent: hexane/ethyl acetate) was required to isolate propargylic ureas 31 and $3 \mathrm{~m}$ with $45 \%$ and $62 \%$ yield, respectively.

General Procedure for the BEMP-Catalyzed Synthesis of Imidazolidin-2-ones 2 (Table 2). A test tube, equipped with a magnetic stir bar, was charged with propargylic urea $1(0.4 \mathrm{mmol})$ and $\mathrm{CH}_{3} \mathrm{CN}(4 \mathrm{~mL})$. BEMP $(5 \mathrm{~mol} \%, 6 \mu \mathrm{L})$ was added. The reaction mixture was stirred at room temperature and monitored by TLC. After completion, the solvent was removed under reduced pressure. The crude was purified by silica gel column chromatography with the mixture hexane/ethyl acetate.

General Procedure for the BEMP-Catalyzed Synthesis of Imidazol-2-ones $\mathbf{4}$ (Table 3). A test tube, equipped with a magnetic stir bar, was charged with propargylic urea $3(0.4 \mathrm{mmol})$ and $\mathrm{CH}_{3} \mathrm{CN}$ $(4 \mathrm{~mL})$. BEMP $(5 \mathrm{~mol} \%, 6 \mu \mathrm{L})$ was added. The reaction mixture was stirred at room temperature for $1 \mathrm{~min}$ (TLC confirmed the completion of the reaction). The solvent was removed under reduced pressure. The crude was purified by silica gel column chromatography with the mixture hexane/ethyl acetate.

One-Pot BEMP-Catalyzed Synthesis of $2 a$ from Propargylic Amine and Isocyanate (Scheme 1a). A test tube, equipped with a magnetic stir bar, was charged with 2-methylbut-3-yn-2-amine (0.4 $\mathrm{mmol}, 33 \mathrm{mg})$, phenyl isocyanate $(0.4 \mathrm{mmol}, 48 \mathrm{mg})$, and $\mathrm{CH}_{3} \mathrm{CN}(4$ $\mathrm{mL})$. BEMP $(5 \mathrm{~mol} \%, 6 \mu \mathrm{L})$ was added. The reaction mixture was stirred at room temperature and monitored by TLC. After completion $(1 \mathrm{~h})$, the solvent was removed under reduced pressure. The crude was purified by silica gel column chromatography (Eluent: hexane/ ethyl acetate 1:1) to afford 2a in quantitative yield ( $81 \mathrm{mg})$.

One-Pot BEMP-Catalyzed Synthesis of $\mathbf{4 a}$ from Propargylic Amine and Isocyanate (Scheme $1 b$ ). A test tube, equipped with a magnetic stir bar, was charged with $\mathrm{N}$-benzyl-1,3-diphenylprop-2-yn1-amine $(0.4 \mathrm{mmol}, 119 \mathrm{mg})$, phenyl isocyanate $(0.4 \mathrm{mmol}, 48 \mathrm{mg})$, and $\mathrm{CH}_{3} \mathrm{CN}(4 \mathrm{~mL})$. BEMP $(5 \mathrm{~mol} \%, 6 \mu \mathrm{L})$ was added. The reaction mixture was stirred at room temperature for $1 \mathrm{~min}$. Solvent was removed under reduced pressure. The crude was purified by silica gel column chromatography (Eluent: hexane/ethyl acetate 6:4) to afford $4 \mathbf{a}$ in $51 \%$ yield $(85 \mathrm{mg})$. With $10 \%$ of BEMP, $4 \mathbf{a}$ was isolated in $62 \%$ yield $(103 \mathrm{mg})$.

Synthesis of $2 n^{\prime} .{ }^{25}$ A dry two-necked flask containing a magnetic stir bar was charged with 1-fluoro-4-isothiocyanatobenzene $(1 \mathrm{mmol}$, $122 \mu \mathrm{L})$, 2-methylbut-3-yn-2-amine ( $1 \mathrm{mmol}, 105 \mu \mathrm{L})$, and dry THF $(10 \mathrm{~mL})$. The resulting mixture was stirred at room temperature and monitored by TLC. After $3 \mathrm{~h}$, the solvent was removed under reduced pressure. The crude did not require purification, and product $\mathbf{2} \mathbf{n}^{\prime}$ was recovered in $98 \%$ yield $(231 \mathrm{mg})$ as a yellow solid.

$\mathrm{N}$-Benzyl-2-methyldodec-3-yn-2-amine. $\mathrm{N}$-Benzyl-2-methyldodec-3-yn-2-amine was synthesized from $2 \mathrm{mmol}$ of starting materials, according to the general procedure. ${ }^{28}$ The crude was purified by flash column chromatography using hexane/ethyl acetate $(9: 1)$ as eluent to give the desired amine $\left(206 \mathrm{mg}, 31 \%\right.$ yield) as a transparent oil. ${ }^{1} \mathrm{H}$ NMR (400 MHz, $\left.\mathrm{CDCl}_{3}\right) \delta 7.40-7.35(\mathrm{~m}, 2 \mathrm{H}), 7.34-7.30(\mathrm{~m}, 2 \mathrm{H})$, 
$7.25-7.22(\mathrm{~m}, 1 \mathrm{H}), 3.88(\mathrm{~s}, 2 \mathrm{H}), 2.23(\mathrm{t}, J=7.0 \mathrm{~Hz}, 2 \mathrm{H}), 1.59-1.49$ $(\mathrm{m}, 2 \mathrm{H}), 1.47-1.42(\mathrm{~m}, 2 \mathrm{H}), 1.40(\mathrm{~s}, 6 \mathrm{H}), 1.34-1.27(\mathrm{~m}, 9 \mathrm{H}), 0.90$ $(\mathrm{t}, J=6.8 \mathrm{~Hz}, 3 \mathrm{H}) \cdot{ }^{13} \mathrm{C}\{1 \mathrm{H}\} \mathrm{NMR}\left(101 \mathrm{MHz}, \mathrm{CDCl}_{3}\right) \delta 141.2$, $128.6,128.5,126.9,85.3,82.4,50.3,49.2,32.0,30.1,29.4,29.2(2 \mathrm{C})$, 29.0, 22.8, 18.8, 14.2. HRMS (ESI) calcd for $\mathrm{C}_{20} \mathrm{H}_{32} \mathrm{~N}(\mathrm{M}+\mathrm{H})^{+} \mathrm{m} / z$ 286.2535, found $m / z 286,2533$.

$\mathrm{N}$-Benzyl-2-methylbut-3-yn-2-amine. N-Benzyl-2-methylbut-3yn-2-amine was synthesized according to the general procedure. ${ }^{27}$ The crude was purified by flash column chromatography using hexane/ethyl acetate $(7: 3)$ as eluent to give the desired amine (476 $\mathrm{mg}, 55 \%$ yield) as a white solid; $\mathrm{mp}$ (hexane) $44.7-45.3{ }^{\circ} \mathrm{C} .{ }^{1} \mathrm{H}$ NMR $\left(300 \mathrm{MHz}, \mathrm{CDCl}_{3}\right) \delta 7.43-7.33(\mathrm{~m}, 4 \mathrm{H}), 7.31-7.26(\mathrm{~m}, 1 \mathrm{H}), 3.91$ $(\mathrm{s}, 2 \mathrm{H}), 2.39(\mathrm{~s}, 1 \mathrm{H}), 1.46$ (two overlapping signals: brs and $\mathrm{s}, 7 \mathrm{H}$ ). Spectroscopic data were consistent with literature values. ${ }^{29}$

$N$-Benzyl-1,3-diphenylprop-2-yn-1-amine. N-Benzyl-1,3-diphenylprop-2-yn-1-amine was synthesized according to the general procedure. $^{28}$ The crude was purified by flash column chromatography using hexane/ethyl acetate $(8: 2)$ as eluent to give the desired amine (349 mg, 59\% yield) as a viscous colorless oil. ${ }^{1} \mathrm{H}$ NMR $(400 \mathrm{MHz}$, $\left.\mathrm{CDCl}_{3}\right) \delta 7.69(\mathrm{~d}, J=7.4 \mathrm{~Hz}, 2 \mathrm{H}), 7.59-7.55(\mathrm{~m}, 2 \mathrm{H}), 7.50-7.41$ $(\mathrm{m}, 5 \mathrm{H}), 7.40-7.36(\mathrm{~m}, 5 \mathrm{H}), 7.35-7.31(\mathrm{~m}, 1 \mathrm{H}), 4.88(\mathrm{~s}, 1 \mathrm{H})$, 4.12-4.00 (m, 2H), 2.01 (bs, $1 \mathrm{H})$. Spectroscopic data were consistent with literature values. ${ }^{30}$

$N$-Benzyl-1-phenylundec-2-yn-1-amine. N-Benzyl-1-phenylundec-2-yn-1-amine was synthesized according to the general procedure. ${ }^{28}$ The crude was purified by flash column chromatography using hexane/ethyl acetate $(9: 1)$ as eluent to give the desired amine (342 mg, 53\% yield) as a yellowish oil. ${ }^{1} \mathrm{H}$ NMR $\left(400 \mathrm{MHz}, \mathrm{CDCl}_{3}\right)$ $\delta 7.63(\mathrm{~d}, J=7.4 \mathrm{~Hz}, 2 \mathrm{H}), 7.48-7.41(\mathrm{~m}, 4 \mathrm{H}), 7.40-7.37(\mathrm{~m}, 2 \mathrm{H})$, 7.36-7.29 (m, 2H), $4.65(\mathrm{~s}, 1 \mathrm{H}), 4.04-3.94(\mathrm{~m}, 2 \mathrm{H}), 2.37$ (further split t, $J=7.0 \mathrm{~Hz}, 2 \mathrm{H}), 1.79$ (bs, $1 \mathrm{H}), 1.70-1.60(\mathrm{~m}, 2 \mathrm{H}), 1.57-1.48$ $(\mathrm{m}, 2 \mathrm{H}), 1.44-1.34(\mathrm{~m}, 8 \mathrm{H}), 0.98(\mathrm{t}, J=6.9 \mathrm{~Hz}, 3 \mathrm{H}) .{ }^{13} \mathrm{C}\{1 \mathrm{H}\}$ NMR $\left(101 \mathrm{MHz}, \mathrm{CDCl}_{3}\right) \delta 141.1,140.1,128.5,128.4,128.2,127.7$, 127.5, 127.0, 86.1, 79.9, 53.3, 51.1, 31.9, 29.3, 29.2, 29.0, 28.9, 22.8, 18.9, 14.2. HRMS (ESI) calcd for $\mathrm{C}_{24} \mathrm{H}_{32} \mathrm{~N}(\mathrm{M}+\mathrm{H})^{+} \mathrm{m} / z$ 334.2534, found $m / z 334.2530$.

$N$-(1,3-Diphenylprop-2-yn-1-yl)butan-1-amine. $N$-Benzyl-1,3-diphenylprop-2-yn-1-amine was synthesized according to the general procedure. ${ }^{28}$ The crude was purified by flash column chromatography using hexane/ethyl acetate $(8: 2)$ as eluent to give the desired amine (315 mg, 60\% yield) as a viscous yellow oil. ${ }^{1} \mathrm{H}$ NMR $(300 \mathrm{MHz}$, $\left.\mathrm{CDCl}_{3}\right) \delta 7.68(\mathrm{~d}, J=7.2 \mathrm{~Hz}, 2 \mathrm{H}), 7.62-7.50(\mathrm{~m}, 2 \mathrm{H}), 7.49-7.40$ $(\mathrm{m}, 2 \mathrm{H}), 7.39-7.32(\mathrm{~m}, 4 \mathrm{H}), 4.88(\mathrm{~s}, 1 \mathrm{H}), 2.98-2.88(\mathrm{~m}, 1 \mathrm{H})$, $2.86-2.70(\mathrm{~m}, 1 \mathrm{H}), 1.64-1.38(\mathrm{~m}, 4 \mathrm{H}), 1.00(\mathrm{t}, J=7.3 \mathrm{~Hz}, 3 \mathrm{H})$. Spectroscopic data were consistent with literature values. ${ }^{31}$

1,3-Diphenyl-N-(1-phenylethyl)prop-2-yn-1-amine. 1,3-Diphenyl-N-(1-phenylethyl)prop-2-yn-1-amine was synthesized according to the general procedure. ${ }^{28}$ The crude was purified by flash column chromatography using hexane/ethyl acetate (9:1) as eluent to give the desired amine ( $257 \mathrm{mg}$, $42 \%$ yield) together with the corresponding imine intermediate. ${ }^{32}{ }^{1} \mathrm{H}$ NMR $\left(400 \mathrm{MHz}, \mathrm{CDCl}_{3}\right) \delta 7.62-7.31(\mathrm{~m}$, $30 \mathrm{H}), 4.71(\mathrm{~s}, 1 \mathrm{H}), 4.50(\mathrm{~s}, 1 \mathrm{H}), 4.40(\mathrm{q}, J=6.6 \mathrm{~Hz}, 1 \mathrm{H}), 3.96(\mathrm{q}, J=$ $6.6 \mathrm{~Hz}, 1 \mathrm{H}), 1.76(\mathrm{brs}, 2 \mathrm{H}), 1.45(\mathrm{~d}, J=6.6 \mathrm{~Hz}, 3 \mathrm{H}), 1.42(\mathrm{~d}, J=6.6$ $\mathrm{Hz}, 3 \mathrm{H})$. Spectroscopic data were consistent with literature values. ${ }^{32}$

$\mathrm{N}$-(4-Methylbenzyl)-1,3-diphenylprop-2-yn-1-amine. $\mathrm{N}$-(4-Methylbenzyl)-1,3-diphenylprop-2-yn-1-amine was synthesized according to the general procedure. ${ }^{28}$ The crude was purified by flash column chromatography using hexane/ethyl acetate (9:1) as eluent to give the desired amine (433 mg, 70\% yield) as a viscous yellow oil. ${ }^{1} \mathrm{H}$ NMR $\left(400 \mathrm{MHz}, \mathrm{CDCl}_{3}\right) \delta 7.78(\mathrm{~d}, J=7.2 \mathrm{~Hz}, 2 \mathrm{H}), 7.69-7.66(\mathrm{~m}, 2 \mathrm{H})$, $7.53(\mathrm{t}, J=7.4 \mathrm{~Hz}, 2 \mathrm{H}), 7.49-7.44(\mathrm{~m}, 6 \mathrm{H}), 7.30(\mathrm{~d}, J=7.8 \mathrm{~Hz}, 2 \mathrm{H})$, $4.96(\mathrm{~s}, 1 \mathrm{H}), 4.12(\mathrm{~s}, 2 \mathrm{H}), 2.49(\mathrm{~s}, 3 \mathrm{H}), 1.98(\mathrm{bs}, 1 \mathrm{H}) .{ }^{13} \mathrm{C}\{1 \mathrm{H}\}$ NMR (101 MHz, $\left.\mathrm{CDCl}_{3}\right) \delta 140.4,136.8,136.6,131.8,129.1,128.5$, $128.4,128.3,128.2,127.8,127.7,123.2,89.4,85.7,53.6,50.9,21.2$. HRMS (ESI) calcd for $\mathrm{C}_{23} \mathrm{H}_{22} \mathrm{~N}(\mathrm{M}+\mathrm{H})^{+} \mathrm{m} / z 312.1752$, found $\mathrm{m} / z$ 312.1754 .

$\mathrm{N}$-(4-Fluorobenzyl)-1,3-diphenylprop-2-yn-1-amine. $\mathrm{N}$-(4-Fluorobenzyl)-1,3-diphenylprop-2-yn-1-amine was synthesized according to the general procedure. ${ }^{28}$ The crude was purified by flash column chromatography using hexane/ethyl acetate (9:1) as eluent to give the desired amine (490 mg, 78\% yield) as a viscous yellowish oil. ${ }^{1} \mathrm{H}$ NMR (400 MHz, $\left.\mathrm{CDCl}_{3}\right) \delta 7.73-7.68(\mathrm{~m}, 2 \mathrm{H}), 7.62-7.57(\mathrm{~m}, 2 \mathrm{H})$, 7.49-7.42 (m, 4H), 7.42-7.36 (m, 4H), 7.13-7.07 (m, 2H), $4.87(\mathrm{~s}$, $1 \mathrm{H}), 4.04(\mathrm{~s}, 2 \mathrm{H}), 1.89(\mathrm{bs}, 1 \mathrm{H}) .{ }^{13} \mathrm{C}\{1 \mathrm{H}\} \operatorname{NMR}\left(101 \mathrm{MHz}, \mathrm{CDCl}_{3}\right)$ $\delta 162.1\left(\mathrm{~d}, J_{\mathrm{C}, \mathrm{F}}=244.8 \mathrm{~Hz}\right), 135.6\left(\mathrm{~d}, J_{\mathrm{C}, \mathrm{F}}=3.1 \mathrm{~Hz}\right), 140.3,131.8$, $130.0\left(\mathrm{~d}, J_{\mathrm{C}, \mathrm{F}}=7.9 \mathrm{~Hz}\right), 128.6,128.4,128.3,127.9,127.7,123.1$, $115.2\left(\mathrm{~d}, J_{\mathrm{C}, \mathrm{F}}=21.2 \mathrm{~Hz}\right), 89.2,85.9,53.6,50.4 .{ }^{19} \mathrm{~F}\{1 \mathrm{H}\} \mathrm{NMR}(376$ $\left.\mathrm{MHz}, \mathrm{CDCl}_{3}\right) \delta-115.56$. HRMS (ESI) calcd for $\mathrm{C}_{22} \mathrm{H}_{18} \mathrm{FN}(\mathrm{M}+$ $\mathrm{H})^{+} \mathrm{m} / z$ 316.1501, found $m / z 316.1498$.

N-Benzyl-3-phenyl-1-(p-tolyl)prop-2-yn-1-amine. N-Benzyl-3phenyl-1-(p-tolyl)prop-2-yn-1-amine was synthesized according to the general procedure. ${ }^{28}$ The crude was purified by flash column chromatography using hexane/ethyl acetate (9:1) as eluent to give the desired amine ( $533 \mathrm{mg}, 86 \%$ yield) as a viscous colorless oil. ${ }^{1} \mathrm{H}$ NMR $\left(400 \mathrm{MHz}, \mathrm{CDCl}_{3}\right) \delta 7.69-7.65(\mathrm{~m}, 2 \mathrm{H}), 7.61-7.56(\mathrm{~m}, 4 \mathrm{H}), 7.53-$ $7.40(\mathrm{~m}, 7 \mathrm{H}), 7.27(\mathrm{~d}, J=7.8 \mathrm{~Hz}, 1 \mathrm{H}), 4.94(\mathrm{~s}, 1 \mathrm{H}), 4.21-4.12(\mathrm{~m}$, $2 \mathrm{H}), 2.53(\mathrm{~s}, 3 \mathrm{H}), 2.11(\mathrm{bs}, 1 \mathrm{H})$. Spectroscopic data were consistent with literature values. ${ }^{33}$

$\mathrm{N}$-Benzyl-1-(4-methoxyphenyl)-3-phenylprop-2-yn-1-amine. $\mathrm{N}$ Benzyl-1-(4-methoxyphenyl)-3-phenylprop-2-yn-1-amine was synthesized according to the general procedure. ${ }^{28}$ The crude was purified by flash column chromatography using hexane/ethyl acetate (8:2) as eluent to give the desired amine $(376 \mathrm{mg}, 58 \%$ yield) as a yellow oil. ${ }^{1} \mathrm{H}$ NMR $\left(400 \mathrm{MHz}, \mathrm{CDCl}_{3}\right) \delta$ 7.68-7.59 (m, 4H), 7.54-7.49 (m, $2 \mathrm{H}), 7.46-7.39(\mathrm{~m}, 5 \mathrm{H}), 7.38-7.33(\mathrm{~m}, 1 \mathrm{H}), 7.04-6.98(\mathrm{~m}, 2 \mathrm{H})$, $4.88(\mathrm{~s}, 1 \mathrm{H}), 4.12-4.03(\mathrm{~m}, 2 \mathrm{H}), 3.88(\mathrm{~s}, 3 \mathrm{H}), 1.94(\mathrm{bs}, 1 \mathrm{H})$. Spectroscopic data were consistent with literature values. ${ }^{30}$

$\mathrm{N}$-Benzyl-1-(3-chlorophenyl)-3-phenylprop-2-yn-1-amine. $\mathrm{N}$ Benzyl-1-(3-chlorophenyl)-3-phenylprop-2-yn-1-amine was synthesized according to the general procedure. ${ }^{28}$ The crude was purified by flash column chromatography using hexane/ethyl acetate (9:1) as eluent to give the desired amine ( $342 \mathrm{mg}, 52 \%$ yield) as dark yellow oil. ${ }^{1} \mathrm{H}$ NMR $\left(300 \mathrm{MHz}, \mathrm{CDCl}_{3}\right) \delta 7.75-7.72(\mathrm{~m}, 1 \mathrm{H}), 7.63-7.58$ (m, 3H), 7.53-7.48 (m, 2H), 7.47-7.40 (m, 5H), 7.39-7.34 (m, $3 \mathrm{H}), 4.87(\mathrm{~s}, 1 \mathrm{H}), 4.07(\mathrm{~s}, 2 \mathrm{H}), 2.03(\mathrm{bs}, 1 \mathrm{H}) .{ }^{13} \mathrm{C}\{1 \mathrm{H}\}$ NMR $(75$ $\left.\mathrm{MHz}, \mathrm{CDCl}_{3}\right) \delta 142.4,139.6,134.4,131.8,129.8,128.6,128.5,128.4$ (2C), 128.0, 127.9, 127.2, 125.9, 122.9, 88.5, 86.3, 53.2, 51.1.HRMS (ESI) calcd for $\mathrm{C}_{22} \mathrm{H}_{18} \mathrm{ClN}(\mathrm{M}+\mathrm{H})^{+} \mathrm{m} / z$ 332.1206, found $\mathrm{m} / z$ 332.1203.

N-Benzyl-3-phenyl-1-(3-vinylphenyl)prop-2-yn-1-amine. N-Benzyl-3-phenyl-1-(3-vinylphenyl)prop-2-yn-1-amine was synthesized according to the general procedure. ${ }^{28}$ The crude was purified by flash column chromatography using hexane/ethyl acetate (9:1) as eluent to give the desired amine $(504 \mathrm{mg}, 78 \%$ yield) as a yellow oil. ${ }^{1} \mathrm{H}$ NMR (400 MHz, $\left.\mathrm{CDCl}_{3}\right) \delta 7.83(\mathrm{~s}, 1 \mathrm{H}), 7.71-7.63(\mathrm{~m}, 3 \mathrm{H})$, $7.57(\mathrm{~d}, J=7.1 \mathrm{~Hz}, 2 \mathrm{H}), 7.53-7.39(\mathrm{~m}, 8 \mathrm{H}), 6.90(\mathrm{dd}, J=17.6,10.9$ $\mathrm{Hz}, 1 \mathrm{H}), 5.94(\mathrm{~d}, J=17.6,1 \mathrm{H}), 5.42(\mathrm{~d}, J=10.9 \mathrm{~Hz}, 1 \mathrm{H}), 4.97(\mathrm{~s}$, $1 \mathrm{H}), 4.19-4.11(\mathrm{~m}, 2 \mathrm{H}), 2.11(\mathrm{bs}, 1 \mathrm{H}) .{ }^{13} \mathrm{C}\{1 \mathrm{H}\}$ NMR $(101 \mathrm{MHz}$, $\left.\mathrm{CDCl}_{3}\right) \delta 140.6,139.8,137.8,136.8,131.8,128.7,128.4(2 \mathrm{C}), 128.3$, 128.2, 127.2, 127.1, 125.7, 125.6, 123.1, 114.1, 89.2, 85.8, 53.6, 51.1. HRMS (ESI) calcd for $\mathrm{C}_{24} \mathrm{H}_{22} \mathrm{~N}(\mathrm{M}+\mathrm{H})^{+} \mathrm{m} / z$ 324.1752, found $\mathrm{m} / z$ 324.1755

N-Benzyl-1-(2-bromophenyl)-3-phenylprop-2-yn-1-amine. $\mathrm{N}$ Benzyl-1-(2-bromophenyl)-3-phenylprop-2-yn-1-amine was synthesized according to the general procedure. ${ }^{28}$ The crude was purified by flash column chromatography using hexane/ethyl acetate $(9: 1)$ as eluent to give the desired amine ( $558 \mathrm{mg}, 74 \%$ yield) as a viscous pale orange oil. ${ }^{1} \mathrm{H}$ NMR $\left(400 \mathrm{MHz}, \mathrm{CDCl}_{3}\right) \delta 7.92(\mathrm{dd}, J=7.7,1.7 \mathrm{~Hz}$, $1 \mathrm{H}), 7.64(\mathrm{dd}, J=8.0,1.2 \mathrm{~Hz}, 1 \mathrm{H}), 7.61-7.56(\mathrm{~m}, 2 \mathrm{H}), 7.50(\mathrm{~d}, J=$ $6.9 \mathrm{~Hz}, 2 \mathrm{H}), 7.42-7.37(\mathrm{~m}, 6 \mathrm{H}), 7.36-7.31(\mathrm{~m}, 1 \mathrm{H}), 7.24-7.19(\mathrm{~m}$, $1 \mathrm{H}), 5.28(\mathrm{~s}, 1 \mathrm{H}), 4.12-4.02(\mathrm{~m}, 2 \mathrm{H}), 2.03(\mathrm{bs}, 1 \mathrm{H}) .{ }^{13} \mathrm{C}\{1 \mathrm{H}\} \mathrm{NMR}$ $\left(101 \mathrm{MHz}, \mathrm{CDCl}_{3}\right) \delta 139.5,139.4,133.2,131.8,129.6,129.4,128.7$, 128.4, 128.3 (2C), 127.8, 127.2, 123.8, 123.0, 88.4, 85.8, 53.7, 51.7. HRMS (ESI) calcd for $\mathrm{C}_{22} \mathrm{H}_{19} \mathrm{BrN}(\mathrm{M}+\mathrm{H})^{+} \mathrm{m} / z$ 376.0700, found $m / z 376.0703$.

N-Benzyl-1-phenyl-3-(p-tolyl)prop-2-yn-1-amine. N-Benzyl-1phenyl-3-( $p$-tolyl)prop-2-yn-1-amine was synthesized according to the general procedure. ${ }^{28}$ The crude was purified by flash column chromatography using hexane/ethyl acetate (9:1) as eluent to give the desired amine ( $280 \mathrm{mg}, 45 \%$ yield) as a viscous yellow oil. ${ }^{1} \mathrm{H}$ NMR 
$\left(400 \mathrm{MHz}, \mathrm{CDCl}_{3}\right) \delta 7.66(\mathrm{~d}, J=7.3 \mathrm{~Hz}, 2 \mathrm{H}), 7.48-7.41(\mathrm{~m}, 5 \mathrm{H})$, $7.40-7.35(\mathrm{~m}, 3 \mathrm{H}), 7.35-7.26(\mathrm{~m}, 2 \mathrm{H}), 7.17(\mathrm{~d}, J=7.9 \mathrm{~Hz}, 2 \mathrm{H})$, $4.84(\mathrm{~s}, 1 \mathrm{H}), 4.08-3.98(\mathrm{~m}, 2 \mathrm{H}), 2.40(\mathrm{~s}, 3 \mathrm{H}), 1.91(\mathrm{bs}, 1 \mathrm{H})$. ${ }^{13} \mathrm{C}\{1 \mathrm{H}\}$ NMR $\left(101 \mathrm{MHz}, \mathrm{CDCl}_{3}\right) \delta 140.5,139.9,138.4,131.8$, $129.2,128.61$ (2C), 128.56, 127.9, 127.8, 127.2, 120.2, 88.5, 86.0, 53.8, 51.2, 21.6. HRMS (ESI) calcd for $\mathrm{C}_{23} \mathrm{H}_{22} \mathrm{~N}(\mathrm{M}+\mathrm{H})^{+} \mathrm{m} / z$ 312.1752, found $m / z 312.1751$.

$N$-Benzyl-3-(4-chlorophenyl)-1-phenylprop-2-yn-1-amine. $\mathrm{N}$ Benzyl-3-(4-chlorophenyl)-1-phenylprop-2-yn-1-amine was synthesized according to the general procedure. ${ }^{28}$ The crude was purified by flash column chromatography using hexane/ethyl acetate $(8: 2)$ as eluent to give the desired amine ( $358 \mathrm{mg}, 54 \%$ yield) as a viscous dark yellow oil. ${ }^{1} \mathrm{H}$ NMR $\left(400 \mathrm{MHz}, \mathrm{CDCl}_{3}\right) \delta 7.64(\mathrm{~d}, J=7.3 \mathrm{~Hz}, 2 \mathrm{H})$, 7.47-7.38 (m,7H), 7.38-7.27 (m, 5H), $4.84(\mathrm{~s}, 1 \mathrm{H}), 4.09-3.94(\mathrm{~m}$, $2 \mathrm{H}), 2.01(\mathrm{bs}, 1 \mathrm{H}) .{ }^{13} \mathrm{C}\{1 \mathrm{H}\} \mathrm{NMR}\left(101 \mathrm{MHz}, \mathrm{CDCl}_{3}\right) \delta 140.2$, $139.8,134.3,133.1,128.8,128.7,128.6,128.5,128.0,127.7,127.2$, 121.6, 90.5, 84.7, 53.8, 51.3. HRMS (ESI) calcd for $\mathrm{C}_{22} \mathrm{H}_{19} \mathrm{ClN}(\mathrm{M}+$ $\mathrm{H})^{+} \mathrm{m} / z$ 332.1206, found $\mathrm{m} / \mathrm{z} 332.1207$.

$N$-Benzyl-3-(4-fluorophenyl)-1-phenylprop-2-yn-1-amine. $N$ Benzyl-3-(4-fluorophenyl)-1-phenylprop-2-yn-1-amine was synthesized according to the general procedure. ${ }^{28}$ The crude was purified by flash column chromatography using hexane/ethyl acetate $(8: 2)$ as eluent to give the desired amine $(265 \mathrm{mg}, 42 \%$ yield) as a viscous yellow oil. ${ }^{1} \mathrm{H}$ NMR $\left(400 \mathrm{MHz}, \mathrm{CDCl}_{3}\right) \delta 7.69-7.62(\mathrm{~m}, 2 \mathrm{H}), 7.54-$ $7.48(\mathrm{~m}, 2 \mathrm{H}), 7.48-7.34(\mathrm{~m}, 7 \mathrm{H}), 7.33-7.28(\mathrm{~m}, 1 \mathrm{H}), 7.10-7.01$ $(\mathrm{m}, 2 \mathrm{H}), 4.84(\mathrm{~s}, 1 \mathrm{H}), 4.11-3.96(\mathrm{~m}, 2 \mathrm{H}), 1.89(\mathrm{~s}, 1 \mathrm{H}) .{ }^{13} \mathrm{C}\{1 \mathrm{H}\}$ $\operatorname{NMR}\left(101 \mathrm{MHz}, \mathrm{CDCl}_{3}\right) \delta 162.51\left(\mathrm{~d}, J_{\mathrm{C}, \mathrm{F}}=249.2 \mathrm{~Hz}\right), 140.35$, $139.84,133.67\left(\mathrm{~d}, J_{\mathrm{C}, \mathrm{F}}=8.3 \mathrm{~Hz}\right), 128.64,128.55,128.51,127.93$, $127.73,127.23,119.27\left(\mathrm{~d}, J_{\mathrm{C}, \mathrm{F}}=3.5 \mathrm{~Hz}\right), 115.62\left(\mathrm{~d}, J_{\mathrm{C}, \mathrm{F}}=22.0 \mathrm{~Hz}\right)$, 89.05, 84.72, 53.73, 51.26. ${ }^{19} \mathrm{~F}\{1 \mathrm{H}\} \operatorname{NMR}\left(376 \mathrm{MHz}, \mathrm{CDCl}_{3}\right) \delta$ -111.03. HRMS (ESI) calcd for $\mathrm{C}_{22} \mathrm{H}_{19} \mathrm{FN}(\mathrm{M}+\mathrm{H})^{+} \mathrm{m} / z$ 316.1501, found $m / z 332.1498$.

1-(2-Methylbut-3-yn-2-yl)-3-phenylurea (1a). Compound 1a was synthesized according to the general procedure. ${ }^{25}$ The crude was dried under vacuum and used without purification. Compound 1a was obtained as a white solid; mp (hexane) $116.3-117.6{ }^{\circ} \mathrm{C} .{ }^{1} \mathrm{H}$ NMR $\left(400 \mathrm{MHz}, \mathrm{DMSO}-d_{6}\right) \delta 8.28(\mathrm{bs}, 1 \mathrm{H}), 7.36$ (d further split, $\left.2 \mathrm{H}\right)$, 7.26-7.17 (m, 2H), 6.95-6.83 (m, 1H), $6.38(\mathrm{bs}, 1 \mathrm{H}), 3.10(\mathrm{~s}, 1 \mathrm{H})$, $1.54(\mathrm{~s}, 6 \mathrm{H}) .{ }^{13} \mathrm{C}\{1 \mathrm{H}\}$ NMR $\left(101 \mathrm{MHz}, \mathrm{DMSO}-d_{6}\right) \delta 153.8,140.2$, 128.6, 121.1, 117.5, 88.5, 70.7, 46.0, 29.3. HRMS (ESI) calcd for $\mathrm{C}_{12} \mathrm{H}_{14} \mathrm{~N}_{2} \mathrm{NaO}(\mathrm{M}+\mathrm{Na})^{+} \mathrm{m} / z$ 225.1004, found $\mathrm{m} / z 225.1008$.

1-(2-Methylbut-3-yn-2-yl)-3-(p-tolyl)urea (1b). Compound $\mathbf{1 b}$ was synthesized according to the general procedure. ${ }^{25}$ The crude was dried under vacuum and used without purification. Compound $\mathbf{1 b}$ was obtained as a white solid; mp (hexane) $144.4-146.1{ }^{\circ} \mathrm{C} .{ }^{1} \mathrm{H}$ NMR (400 MHz, DMSO- $\left.d_{6}\right) \delta 8.19$ (bs, $\left.1 \mathrm{H}\right), 7.25(\mathrm{~d}, J=8.4 \mathrm{~Hz}$, $2 \mathrm{H}), 7.03(\mathrm{~d}, J=8.3 \mathrm{~Hz}, 2 \mathrm{H}), 6.34(\mathrm{bs}, 1 \mathrm{H}), 3.11(\mathrm{~s}, 1 \mathrm{H}), 2.22(\mathrm{~s}$, $3 \mathrm{H}), 1.54(\mathrm{~s}, 6 \mathrm{H}) .{ }^{13} \mathrm{C}\{1 \mathrm{H}\}$ NMR (101 MHz, DMSO-d 6 ) $\delta 153.9$, 137.7, 129.8, 129.0, 117.6, 88.7, 70.7, 46.0, 29.4, 20.3. HRMS (ESI) calcd for $\mathrm{C}_{13} \mathrm{H}_{16} \mathrm{~N}_{2} \mathrm{NaO}(\mathrm{M}+\mathrm{Na})^{+} \mathrm{m} / z$ 239.1160, found $\mathrm{m} / \mathrm{z}$ 239.1158.

1-(4-Fluorophenyl)-3-(2-methylbut-3-yn-2-yl)urea (1c). Compound $1 \mathrm{c}$ was synthesized according to the general procedure. ${ }^{25}$ The crude was dried under vacuum and used without purification. Compound 1c was obtained as a light purple solid; mp (hexane) 174.1-174.8 ${ }^{\circ} \mathrm{C} .{ }^{1} \mathrm{H}$ NMR (400 MHz, DMSO- $\left.d_{6}\right) \delta 8.33$ (bs, $\left.1 \mathrm{H}\right)$, 7.41-7.30 (m, 2H), 7.09-7.01 (m, 2H), $6.38(\mathrm{bs}, 1 \mathrm{H}), 3.11(\mathrm{~s}, 1 \mathrm{H})$, $1.53(\mathrm{~s}, 6 \mathrm{H}) .{ }^{13} \mathrm{C}\{1 \mathrm{H}\}$ NMR $\left(101 \mathrm{MHz}, \mathrm{DMSO}-d_{6}\right) \delta 156.9\left(\mathrm{~d}, J_{\mathrm{C}, \mathrm{F}}=\right.$ $237.2 \mathrm{~Hz}), 153.9,136.6\left(\mathrm{~d}, J_{\mathrm{C}, \mathrm{F}}=2.3 \mathrm{~Hz}\right), 119.1\left(\mathrm{~d}, J_{\mathrm{C}, \mathrm{F}}=7.5 \mathrm{~Hz}\right)$, $115.1\left(\mathrm{~d}, J_{\mathrm{C}, \mathrm{F}}=22.0 \mathrm{~Hz}\right), 88.6,70.8,46.0,29.4 .{ }^{19} \mathrm{~F}\{1 \mathrm{H}\}$ NMR $(376$ $\left.\mathrm{MHz}, \mathrm{DMSO}-d_{6}\right) \delta-122.40$. HRMS (ESI) calcd for $\mathrm{C}_{12} \mathrm{H}_{13} \mathrm{FN}_{2} \mathrm{NaO}$ $(\mathrm{M}+\mathrm{Na})^{+} \mathrm{m} / z$ 243.0910, found $\mathrm{m} / z$ 243.0912.

Methyl 4-(3-(2-Methylbut-3-yn-2-yl)ureido)benzoate (1d). Compound $1 \mathbf{d}$ was synthesized according to the general procedure. ${ }^{25}$ The crude was dried under vacuum and used without purification. Compound 1d was obtained as a white solid; mp (hexane) 189.6$190.9^{\circ} \mathrm{C} .{ }^{1} \mathrm{H}$ NMR (400 MHz, DMSO-d $\left.d_{6}\right) \delta 8.73$ (bs, $\left.1 \mathrm{H}\right), 7.86-$ $7.81(\mathrm{~m}, 2 \mathrm{H}), 7.52-7.48(\mathrm{~m}, 2 \mathrm{H}), 6.58(\mathrm{bs}, 1 \mathrm{H}), 3.79(\mathrm{~s}, 3 \mathrm{H}), 3.10$ $(\mathrm{s}, 1 \mathrm{H}), 1.55(\mathrm{~s}, 6 \mathrm{H}) .{ }^{13} \mathrm{C}\{1 \mathrm{H}\} \mathrm{NMR}\left(101 \mathrm{MHz}, \mathrm{DMSO}-d_{6}\right) \delta 166.0$, $153.4,144.9,130.4,121.9,116.7,88.3,70.8,51.6,46.2$, 29.2. HRMS
(ESI) calcd for $\mathrm{C}_{14} \mathrm{H}_{16} \mathrm{~N}_{2} \mathrm{NaO}_{3}(\mathrm{M}+\mathrm{Na})^{+} \mathrm{m} / z$ 283.1059, found $m / z$ 283.1057.

1-(3-Ethylpent-1-yn-3-yl)-3-phenylurea (1e). Compound 1e was synthesized according to the general procedure. ${ }^{25}$ The crude was dried under vacuum and used without purification. Compound le was obtained as a white solid; mp (hexane) $122.3-123.1{ }^{\circ} \mathrm{C} .{ }^{1} \mathrm{H}$ NMR $\left(400 \mathrm{MHz}, \mathrm{CDCl}_{3}\right) \delta 7.98(\mathrm{bs}, 1 \mathrm{H}), 7.35(\mathrm{~d}, J=7.6 \mathrm{~Hz}, 2 \mathrm{H}), 7.21(\mathrm{t}$, $J=7.9 \mathrm{~Hz}, 2 \mathrm{H}), 6.97(\mathrm{t}, J=7.4 \mathrm{~Hz}, 1 \mathrm{H}), 5.85(\mathrm{bs}, 1 \mathrm{H}), 2.32(\mathrm{~s}, 1 \mathrm{H})$, 2.01-1.78 (m, 2H), 1.76-1.53 (m, 2H), $0.99(\mathrm{t}, J=7.4 \mathrm{~Hz}, 6 \mathrm{H})$. ${ }^{13} \mathrm{C}\{1 \mathrm{H}\}$ NMR $\left(101 \mathrm{MHz}, \mathrm{CDCl}_{3}\right) \delta 155.5,139.2,128.9,122.8$, $119.8,85.8,71.8,55.6,31.4$, 8.5. HRMS (ESI) calcd for $\mathrm{C}_{14} \mathrm{H}_{18} \mathrm{~N}_{2} \mathrm{NaO}(\mathrm{M}+\mathrm{Na})^{+} \mathrm{m} / z$ 253.1317, found $\mathrm{m} / z 253.1320$.

1-(1-Ethynylcyclohexyl)-3-phenylurea (1f). Compound 1f was synthesized according to the general procedure. ${ }^{25}$ The crude was dried under vacuum and used without purification. Compound If was obtained as a white solid; mp (hexane) $157.2-157.8{ }^{\circ} \mathrm{C} .{ }^{1} \mathrm{H}$ NMR $\left(400 \mathrm{MHz}, \mathrm{DMSO}-d_{6}\right) \delta 8.32(\mathrm{bs}, 1 \mathrm{H}), 7.36(\mathrm{~d}, J=7.6 \mathrm{~Hz}, 2 \mathrm{H}), 7.22$ $(\mathrm{t}, J=7.9 \mathrm{~Hz}, 2 \mathrm{H}), 6.89(\mathrm{t}, J=7.3 \mathrm{~Hz}, 1 \mathrm{H}), 6.29(\mathrm{bs}, 1 \mathrm{H}), 3.15(\mathrm{~s}$, $1 \mathrm{H}), 2.08-1.92(\mathrm{~m}, 2 \mathrm{H}), 1.81-1.69(\mathrm{~m}, 2 \mathrm{H}), 1.62-1.43(\mathrm{~m}, 5 \mathrm{H})$, $1.34-1.22(\mathrm{~m}, 1 \mathrm{H}) .{ }^{13} \mathrm{C}\{1 \mathrm{H}\}$ NMR (101 MHz, DMSO-d 6 ) $\delta 154.2$, 140.8, 129.1, 121.5, 117.9, 87.6, 73.3, 50.6, 37.3, 25.3, 22.4. HRMS (ESI) calcd for $\mathrm{C}_{15} \mathrm{H}_{18} \mathrm{~N}_{2} \mathrm{NaO}(\mathrm{M}+\mathrm{Na})^{+} \mathrm{m} / z 265.1317$, found $\mathrm{m} / z$ 265.1319.

1-Benzyl-1-(2-methylbut-3-yn-2-yl)-3-phenylurea (1g). Compound $1 \mathrm{~g}$ was synthesized according to the general procedure. ${ }^{27}$ Compound $1 \mathrm{~g}$ was obtained as colorless oil; ${ }^{1} \mathrm{H}$ NMR $(300 \mathrm{MHz}$, $\left.\mathrm{CDCl}_{3}\right) \delta 7.47-7.41(\mathrm{~m}, 4 \mathrm{H}), 7.37-7.31(\mathrm{~m}, 2 \mathrm{H}), 7.29-7.23(\mathrm{~m}$, $4 \mathrm{H}), 6.95(\mathrm{bs}, 1 \mathrm{H}), 4.85(\mathrm{~s}, 2 \mathrm{H}), 2.56(\mathrm{~s}, 1 \mathrm{H}), 1.88(\mathrm{~s}, 6 \mathrm{H}) .{ }^{13} \mathrm{C}\{1 \mathrm{H}\}$ NMR $\left(101 \mathrm{MHz}, \mathrm{CDCl}_{3}\right) \delta 156.2,139.3,139.2,129.2,128.9,127.6$, $126.5,123.1,119.8,88.1,72.8,54.8,50.1,29.2$. HRMS (ESI) calcd for $\mathrm{C}_{19} \mathrm{H}_{20} \mathrm{~N}_{2} \mathrm{NaO}(\mathrm{M}+\mathrm{Na})^{+} m / z$ 315.1473, found $m / z 315.1476$.

1-Benzyl-1-(2-methyldodec-3-yn-2-yl)-3-phenylurea (1h). Compound $1 \mathrm{~h}$ was synthesized according to the general procedure. ${ }^{26}$ The crude was purified by silica gel column chromatography to obtain $\mathbf{1 h}$ with a $78 \%$ yield $(352 \mathrm{mg})$ as a yellowish oil. ${ }^{1} \mathrm{H}$ NMR $(400 \mathrm{MHz}$, $\left.\mathrm{CDCl}_{3}\right) \delta 7.51(\mathrm{bs}, 1 \mathrm{H}), 7.44-7.34(\mathrm{~m}, 4 \mathrm{H}), 7.33-7.24(\mathrm{~m}, 5 \mathrm{H})$, $7.05-6.98(\mathrm{~m}, 1 \mathrm{H}), 4.87(\mathrm{~s}, 2 \mathrm{H}), 2.21(\mathrm{t}, J=7.0 \mathrm{~Hz}, 2 \mathrm{H}), 1.80(\mathrm{~s}$, $6 \mathrm{H}), 1.49-1.39(\mathrm{~m}, 2 \mathrm{H}), 1.37-1.19(\mathrm{~m}, 10 \mathrm{H}), 0.91(\mathrm{t}, J=7.0 \mathrm{~Hz}$, $3 \mathrm{H}) .{ }^{13} \mathrm{C}\{1 \mathrm{H}\} \mathrm{NMR}\left(101 \mathrm{MHz}, \mathrm{CDCl}_{3}\right) \delta 156.4,140.1,139.5,128.9$, $128.8,127.2,126.6,122.8,119.6,85.9,84.6,54.6,49.4,31.9,29.8$, 29.3, 29.2, 29.0, 28.6, 22.8, 18.8, 14.2. HRMS (ESI) calcd for $\mathrm{C}_{27} \mathrm{H}_{37} \mathrm{~N}_{2} \mathrm{O}(\mathrm{M}+\mathrm{H})^{+} \mathrm{m} / z$ 405.2906, found $\mathrm{m} / z$ 405.2908.

1-(2-Methyl-4-phenylbut-3-yn-2-yl)-3-phenylurea (1i). Compound $1 \mathbf{i}$ was synthesized according to the general procedure. ${ }^{26}$ The crude was purified by silica gel column chromatography to obtain 1i with a $78 \%$ yield $(217 \mathrm{mg})$ as a yellowish solid; $\mathrm{mp}$ (hexane) $182.3-183.7^{\circ} \mathrm{C} .{ }^{1} \mathrm{H}$ NMR $\left(400 \mathrm{MHz}\right.$, DMSO- $\left.d_{6}\right) \delta 8.36(\mathrm{bs}, 1 \mathrm{H})$, $7.45-7.30(\mathrm{~m}, 7 \mathrm{H}), 7.23(\mathrm{t}, J=7.9 \mathrm{~Hz}, 2 \mathrm{H}), 6.90(\mathrm{t}, J=7.3 \mathrm{~Hz}, 1 \mathrm{H})$, $6.53(\mathrm{bs}, 1 \mathrm{H}), 1.66(\mathrm{~s}, 6 \mathrm{H}) .{ }^{13} \mathrm{C}\{1 \mathrm{H}\} \mathrm{NMR}\left(101 \mathrm{MHz}, \mathrm{DMSO}-d_{6}\right) \delta$ 153.8, 140.2, 131.2, 128.6, 128.4, 128.1, 122.6, 121.0, 117.5, 94.5, 79.6, 46.6, 29.4. HRMS (ESI) calcd for $\mathrm{C}_{18} \mathrm{H}_{18} \mathrm{~N}_{2} \mathrm{NaO}(\mathrm{M}+\mathrm{Na})^{+} \mathrm{m} /$ $z$ 301.1317, found $m / z 301.1313$.

1-(2-Methyl-4-(4-(trifluoromethyl)phenyl)but-3-yn-2-yl)-3-phenylurea (1j). Compound $\mathbf{1 j}$ was synthesized according to the general procedure. $^{26}$ The crude was purified by silica gel column chromatography to obtain $\mathbf{1 j}$ with a $91 \%$ yield $(315 \mathrm{mg})$ as a white solid; mp (hexane) $183.9-185.2^{\circ} \mathrm{C} .{ }^{1} \mathrm{H}$ NMR (400 MHz, DMSO- $\left.d_{6}\right)$ $\delta 8.38(\mathrm{bs}, 1 \mathrm{H}), 7.68(\mathrm{~d}, J=8.3 \mathrm{~Hz}, 2 \mathrm{H}), 7.58(\mathrm{~d}, J=8.2 \mathrm{~Hz}, 2 \mathrm{H})$, $7.43(\mathrm{~d}, J=7.6 \mathrm{~Hz}, 2 \mathrm{H}), 7.23(\mathrm{t}, J=7.9 \mathrm{~Hz}, 2 \mathrm{H}), 6.90(\mathrm{t}, J=7.3 \mathrm{~Hz}$, $1 \mathrm{H}), 6.58(\mathrm{bs}, 1 \mathrm{H}), 1.68(\mathrm{~s}, 6 \mathrm{H}) .{ }^{13} \mathrm{C}\{1 \mathrm{H}\}$ NMR $(101 \mathrm{MHz}$, DMSO$\left.d_{6}\right) \delta 153.9,140.2,132.0,128.6,128.3\left(\mathrm{q}, J_{\mathrm{C}, \mathrm{F}}=32.1 \mathrm{~Hz}\right), 127.1(\mathrm{q}$, $\left.J_{\mathrm{C}, \mathrm{F}}=1.2 \mathrm{~Hz}\right), 125.4\left(\mathrm{q}, J_{\mathrm{C}, \mathrm{F}}=3.6 \mathrm{~Hz}\right), 124.0\left(\mathrm{q}, J_{\mathrm{C}, \mathrm{F}}=271.9 \mathrm{~Hz}\right)$, 121.2, 117.7, 97.4, 78.5, 46.6, 29.3. ${ }^{19} \mathrm{~F}\{1 \mathrm{H}\}$ NMR (376 MHz, DMSO-d $\left.d_{6}\right) \delta-61.47$. HRMS (ESI) calcd for $\mathrm{C}_{19} \mathrm{H}_{17} \mathrm{~F}_{3} \mathrm{~N}_{2} \mathrm{NaO}(\mathrm{M}+$ $\mathrm{Na})^{+} \mathrm{m} / z$ 369.1191, found $\mathrm{m} / z$ 369.1194.

1-(4-(4-Methoxyphenyl)-2-methylbut-3-yn-2-yl)-3-phenylurea $(1 \mathrm{k})$. Compound $1 \mathrm{k}$ was synthesized according to the general procedure. $^{26}$ The crude was purified by silica gel column chromatography to obtain $1 \mathrm{k}$ with $57 \%$ yield $(176 \mathrm{mg})$ as pale yellow solid; mp (hexane) $178.2-178.6{ }^{\circ} \mathrm{C} .{ }^{1} \mathrm{H}$ NMR (400 MHz, 
DMSO- $\left.d_{6}\right) \delta 8.35(\mathrm{bs}, 1 \mathrm{H}), 7.41(\mathrm{~d}, J=7.8 \mathrm{~Hz}, 2 \mathrm{H}), 7.32(\mathrm{~d}, J=8.7$ $\mathrm{Hz}, 2 \mathrm{H}), 7.23(\mathrm{t}, J=7.8 \mathrm{~Hz}, 2 \mathrm{H}), 6.95-6.86(\mathrm{~m}, 3 \mathrm{H}), 6.51(\mathrm{bs}, 1 \mathrm{H})$, $3.74(\mathrm{~s}, 3 \mathrm{H}), 1.66(\mathrm{~s}, 6 \mathrm{H}) .{ }^{13} \mathrm{C}\{1 \mathrm{H}\}$ NMR $\left(101 \mathrm{MHz}, \mathrm{DMSO}-d_{6}\right) \delta$ $159.1,153.9,140.3,132.8,128.7,121.1,117.6,114.7,114.1,93.0$, 79.6, 55.1, 46.8, 29.6. HRMS (ESI) calcd for $\mathrm{C}_{19} \mathrm{H}_{20} \mathrm{~N}_{2} \mathrm{NaO}_{2}$ (M $+\mathrm{Na})^{+} \mathrm{m} / z$ 331.1422, found $\mathrm{m} / z$ 331.1424.

1-(2-Methyl-4-(thiophen-2-yl)but-3-yn-2-yl)-3-phenylurea (1I). Compound $\mathbf{1 l}$ was synthesized according to the general procedure. ${ }^{26}$ The crude was purified by silica gel column chromatography to obtain 11 with $94 \%$ yield $(267 \mathrm{mg}$ ) as a yellowish solid; $\mathrm{mp}$ (hexane) 210.3 $211.4{ }^{\circ} \mathrm{C} .{ }^{1} \mathrm{H}$ NMR $\left(400 \mathrm{MHz}, \mathrm{DMSO}-d_{6}\right) \delta 8.36(\mathrm{bs}, 1 \mathrm{H}), 7.51(\mathrm{~d}, J$ $=4.9 \mathrm{~Hz}, 1 \mathrm{H}), 7.41(\mathrm{~d}, J=7.9 \mathrm{~Hz}, 2 \mathrm{H}), 7.26-7.18(\mathrm{~m}, 3 \mathrm{H}), 7.04-$ $6.99(\mathrm{~m}, 1 \mathrm{H}), 6.91(\mathrm{t}, J=7.3 \mathrm{~Hz}, 1 \mathrm{H}), 6.55(\mathrm{bs}, 1 \mathrm{H}), 1.65(\mathrm{~s}, 6 \mathrm{H})$. ${ }^{13} \mathrm{C}\{1 \mathrm{H}\}$ NMR $\left(101 \mathrm{MHz}, \mathrm{DMSO}-d_{6}\right) \delta 153.9,140.2,131.8,128.7$, 127.7, 127.4, 122.4, 121.2, 117.6, 98.3, 73.1, 46.8, 29.4. HRMS (ESI) calcd for $\mathrm{C}_{16} \mathrm{H}_{16} \mathrm{~N}_{2} \mathrm{NaOS}(\mathrm{M}+\mathrm{Na})^{+} \mathrm{m} / z$ 307.0881, found $\mathrm{m} / \mathrm{z}$ 307.0883 .

Ethyl 2-(3-(2-Methylbut-3-yn-2-yl)ureido)acetate (1m). Compound $1 \mathrm{~m}$ was synthesized according to the general procedure. ${ }^{25}$ The crude was dried under vacuum and used without purification. Compound $1 \mathrm{~m}$ was obtained as a white solid; mp (hexane) 83.2$84.8{ }^{\circ} \mathrm{C} .{ }^{1} \mathrm{H}$ NMR $\left(400 \mathrm{MHz}, \mathrm{CDCl}_{3}\right) \delta 4.19(\mathrm{q}, J=7.1 \mathrm{~Hz}, 2 \mathrm{H})$, $4.01(\mathrm{~s}, 2 \mathrm{H}), 2.42(\mathrm{~s}, 1 \mathrm{H}), 1.59(\mathrm{~s}, 6 \mathrm{H}), 1.27(\mathrm{t}, J=7.1 \mathrm{~Hz}, 3 \mathrm{H})$. ${ }^{13} \mathrm{C}\{1 \mathrm{H}\} \mathrm{NMR}\left(101 \mathrm{MHz}, \mathrm{CDCl}_{3}\right) \delta 171.3,157.2,87.5,70.4,61.4$, 46.9, 42.2, 30.1, 14.3. HRMS (ESI) calcd for $\mathrm{C}_{10} \mathrm{H}_{16} \mathrm{~N}_{2} \mathrm{NaO}_{3}$ (M $+\mathrm{Na})^{+} \mathrm{m} / z$ 235.1059, found $\mathrm{m} / z$ 235.1058.

1-Benzyl-1-(1,3-diphenylprop-2-yn-1-yl)-3-phenylurea (3a). Compound $3 \mathbf{a}$ was synthesized according to the general procedure. ${ }^{25}$ The crude was dried under vacuum and used without purification. Compound 3a was obtained as a yellow solid; mp (hexane) 96.2$97.7^{\circ} \mathrm{C}$. The spectroscopic data of $3 \mathbf{a}$ were consistent with literature

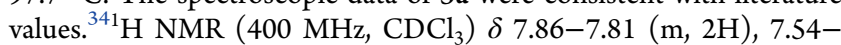
$7.37(\mathrm{~m}, 13 \mathrm{H}), 7.33-7.25(\mathrm{~m}, 4 \mathrm{H}), 7.15(\mathrm{~s}, 1 \mathrm{H}), 7.12-7.03(\mathrm{~m}, 1 \mathrm{H})$, 6.59 (bs, $1 \mathrm{H}), 4.76(\mathrm{~d}, J=17.1 \mathrm{~Hz}, 1 \mathrm{H}), 4.57(\mathrm{~d}, J=17.1 \mathrm{~Hz}, 1 \mathrm{H})$. ${ }^{13} \mathrm{C}\{1 \mathrm{H}\}$ NMR $\left(101 \mathrm{MHz}, \mathrm{CDCl}_{3}\right) \delta 155.5,138.7,138.0,137.1$, 131.6, 129.1, 128.7, 128.6, 128.5, 128.4, 128.3, 128.2, 128.0, 127.7, 127.0, 123.1, 122.3, 119.7, 86.9, 86.0, 51.3, 48.5. HRMS (ESI) calcd for $\mathrm{C}_{29} \mathrm{H}_{25} \mathrm{~N}_{2} \mathrm{O}(\mathrm{M}+\mathrm{H})^{+} \mathrm{m} / z$ 417.1967, found $\mathrm{m} / z$ 417.1970.

1-Benzyl-3-phenyl-1-(1-phenylundec-2-yn-1-yl)urea (3b). Compound $\mathbf{3 b}$ was synthesized according to the general procedure. ${ }^{25}$ The crude was dried under vacuum and used without purification. Compound $\mathbf{3 b}$ was obtained as an orange oil. ${ }^{1} \mathrm{H}$ NMR $(400 \mathrm{MHz}$, $\left.\mathrm{CDCl}_{3}\right) \delta 7.72(\mathrm{~d}, J=7.5 \mathrm{~Hz}, 2 \mathrm{H}), 7.48-7.35(\mathrm{~m}, 8 \mathrm{H}), 7.33-7.12$ $(\mathrm{m}, 4 \mathrm{H}), 7.03(\mathrm{t}, J=7.1 \mathrm{~Hz}, 1 \mathrm{H}), 6.75(\mathrm{~s}, 1 \mathrm{H}), 6.52(\mathrm{~s}, 1 \mathrm{H}), 4.68(\mathrm{~d}$, $J=16.8 \mathrm{~Hz}, 1 \mathrm{H}), 4.48(\mathrm{~d}, J=16.9 \mathrm{~Hz}, 1 \mathrm{H}), 2.35(\mathrm{t}, J=6.7 \mathrm{~Hz}, 2 \mathrm{H})$, $1.63-1.52(\mathrm{~m}, 2 \mathrm{H}), 1.51-1.25(\mathrm{~m}, 10 \mathrm{H}), 0.99(\mathrm{t}, J=6.8 \mathrm{~Hz}, 3 \mathrm{H})$. ${ }^{13} \mathrm{C}\{1 \mathrm{H}\}$ NMR $\left(101 \mathrm{MHz}, \mathrm{CDCl}_{3}\right) \delta 155.5,138.8,138.6,137.4$, 128.9, 128.7, 128.6, 128.0, 127.9, 127.6, 127.2, 123.0, 119.7, 87.8, 76.7, 51.1, 48.6, 31.8, 29.2, 29.1, 28.9, 28.5, 22.6, 18.7, 14.1. HRMS (ESI) calcd for $\mathrm{C}_{31} \mathrm{H}_{37} \mathrm{~N}_{2} \mathrm{O}(\mathrm{M}+\mathrm{H})^{+} \mathrm{m} / z$ 453.2906, found $\mathrm{m} / z$ 453.2909.

1-Butyl-1-(1,3-diphenylprop-2-yn-1-yl)-3-phenylurea (3c). Compound $3 \mathrm{c}$ was synthesized according to the general procedure. ${ }^{25}$ The crude was dried under vacuum and used without purification. Compound $3 \mathrm{c}$ was obtained as a yellowish oil. ${ }^{1} \mathrm{H}$ NMR $(400 \mathrm{MHz}$, $\left.\mathrm{CDCl}_{3}\right) \delta 7.69-7.64(\mathrm{~m}, 2 \mathrm{H}), 7.55-7.50(\mathrm{~m}, 2 \mathrm{H}), 7.44-7.33(\mathrm{~m}$, $8 \mathrm{H}), 7.32-7.27(\mathrm{~m}, 2 \mathrm{H}), 7.08-7.02(\mathrm{~m}, 1 \mathrm{H}), 6.67(\mathrm{~s}, 1 \mathrm{H}), 6.58(\mathrm{~s}$, $1 \mathrm{H}), 3.48-3.30(\mathrm{~m}, 2 \mathrm{H}), 1.87-1.73(\mathrm{~m}, 1 \mathrm{H}), 1.61-1.43(\mathrm{~m}, 1 \mathrm{H})$, $1.42-1.26(\mathrm{~m}, 2 \mathrm{H}), 0.91(\mathrm{t}, J=7.3 \mathrm{~Hz}, 3 \mathrm{H}) .{ }^{13} \mathrm{C}\{1 \mathrm{H}\}$ NMR $(101$ $\left.\mathrm{MHz}, \mathrm{CDCl}_{3}\right) \delta 154.9,139.0,138.1,131.8,129.0,128.8,128.7,128.5$, 128.2, 127.5, 123.2, 122.6, 120.0, 86.8, 86.1, 51.3, 45.5, 31.5, 20.4, 13.9. HRMS (ESI) calcd for $\mathrm{C}_{26} \mathrm{H}_{27} \mathrm{~N}_{2} \mathrm{O}(\mathrm{M}+\mathrm{H})^{+} \mathrm{m} / z$ 383.2123, found $m / z 383.2120$.

1-(1,3-Diphenylprop-2-yn-1-yl)-3-phenyl-1-(1-phenylethyl)urea (3d). Compound $\mathbf{3 d}$ was synthesized according to the general procedure. ${ }^{25}$ Compound $3 \mathrm{~d}$ was obtained as a colorless oil. ${ }^{1} \mathrm{H}$ NMR $\left(400 \mathrm{MHz}, \mathrm{CDCl}_{3}\right) \delta 7.91(\mathrm{~d}, J=7.6 \mathrm{~Hz}, 2 \mathrm{H}), 7.79(\mathrm{~d}, J=8.0 \mathrm{~Hz}$, $2 \mathrm{H}), 7.55-7.45(\mathrm{~m}, 4 \mathrm{H}), 7.45-7.30(\mathrm{~m}, 6 \mathrm{H}), 7.29-7.22(\mathrm{~m}, 3 \mathrm{H})$, $7.14(\mathrm{t}, J=7.8 \mathrm{~Hz}, 2 \mathrm{H}), 7.03(\mathrm{t}, J=7.3 \mathrm{~Hz}, 1 \mathrm{H}), 6.92(\mathrm{bs}, 1 \mathrm{H}), 6.36$ $(\mathrm{s}, 1 \mathrm{H}), 5.52-5.48(\mathrm{~m}, 1 \mathrm{H}), 1.44(\mathrm{~d}, J=7.1 \mathrm{~Hz}, 3 \mathrm{H}) .{ }^{13} \mathrm{C}\{1 \mathrm{H}\} \mathrm{NMR}$ $\left(101 \mathrm{MHz}, \mathrm{CDCl}_{3}\right) \delta 154.6,140.9,138.8,138.2,131.6,129.0,128.7$ (2C), 128.6, 128.45, 128.36, 128.3, 128.1, 127.7, 127.2, 122.9, 119.4, 86.9, 86.5, 52.0, 50.7, 17.2. HRMS (ESI) calcd for $\mathrm{C}_{30} \mathrm{H}_{27} \mathrm{~N}_{2} \mathrm{O}(\mathrm{M}+$ $\mathrm{H})^{+} \mathrm{m} / z$ 431.2123, found $\mathrm{m} / \mathrm{z} 431.2121$.

1-(1,3-Diphenylprop-2-yn-1-yl)-1-(4-methylbenzyl)-3-phenylurea (3e). Compound $3 \mathrm{e}$ was synthesized according to the general procedure. ${ }^{25}$ Compound $3 \mathbf{e}$ was obtained as an orange solid; $\mathrm{mp}$ (hexane) $109.8-111.2^{\circ} \mathrm{C} .{ }^{1} \mathrm{H} \mathrm{NMR}\left(400 \mathrm{MHz}, \mathrm{CDCl}_{3}\right) \delta 7.85(\mathrm{~d}, J$ $=7.5 \mathrm{~Hz}, 2 \mathrm{H}), 7.55-7.48(\mathrm{~m}, 4 \mathrm{H}), 7.45(\mathrm{~d}, J=7.2 \mathrm{~Hz}, 1 \mathrm{H}), 7.41-$ $7.35(\mathrm{~m}, 5 \mathrm{H}), 7.32-7.29(\mathrm{~m}, 4 \mathrm{H}), 7.26(\mathrm{~d}, J=7.9 \mathrm{~Hz}, 2 \mathrm{H}), 7.18(\mathrm{~s}$, $1 \mathrm{H}), 7.10-7.04(\mathrm{~m}, 1 \mathrm{H}), 6.67$ (bs, $1 \mathrm{H}), 4.72(\mathrm{~d}, J=16.9 \mathrm{~Hz}, 1 \mathrm{H})$, $4.52(\mathrm{~d}, J=16.9 \mathrm{~Hz}, 1 \mathrm{H}), 2.43(\mathrm{~s}, 3 \mathrm{H}) .{ }^{13} \mathrm{C}\{1 \mathrm{H}\}$ NMR $(101 \mathrm{MHz}$, $\left.\mathrm{CDCl}_{3}\right) \delta 155.5,138.8,138.0,137.6,134.0,131.6,129.7,128.7,128.6$, 128.5 , 128.2, 128.1, 127.6, 127.0, 123.0, 122.3, 119.6, 86.8, 86.1, 51.2, 48.1, 21.0. HRMS (ESI) calcd for $\mathrm{C}_{30} \mathrm{H}_{27} \mathrm{~N}_{2} \mathrm{O}(\mathrm{M}+\mathrm{H})^{+} \mathrm{m} / z$ 431.2123, found $m / z 431.2119$.

1-(1,3-Diphenylprop-2-yn-1-yl)-1-(4-fluorobenzyl)-3-phenylurea (3f). Compound $3 \mathrm{f}$ was synthesized according to the general procedure. ${ }^{25}$ Compound $3 \mathrm{f}$ was obtained as a light orange solid; $\mathrm{mp}$ (hexane) $125.6-126.8{ }^{\circ} \mathrm{C} .{ }^{1} \mathrm{H}$ NMR $\left(400 \mathrm{MHz}, \mathrm{CDCl}_{3}\right) \delta 7.76$ $(\mathrm{d}, J=7.6 \mathrm{~Hz}, 2 \mathrm{H}), 7.51-7.45(\mathrm{~m}, 4 \mathrm{H}), 7.44-7.36(\mathrm{~m}, 6 \mathrm{H}), 7.32-$ $7.23(\mathrm{~m}, 4 \mathrm{H}), 7.13-7.04(\mathrm{~m}, 3 \mathrm{H}), 7.00(\mathrm{~s}, 1 \mathrm{H}), 6.53$ (bs, $1 \mathrm{H}), 4.70$ $(\mathrm{d}, J=17.0 \mathrm{~Hz}, 1 \mathrm{H}), 4.54(\mathrm{~d}, J=17.0 \mathrm{~Hz}, 1 \mathrm{H}) .{ }^{13} \mathrm{C}\{1 \mathrm{H}\} \mathrm{NMR}(101$ $\left.\mathrm{MHz} \mathrm{CDCl}_{3}\right) \delta 162.4(\mathrm{~d}, J=246.7 \mathrm{~Hz}), 155.4,138.7,137.9,133.0$ $(\mathrm{d}, J=3.1 \mathrm{~Hz}), 131.7,128.9$ (d, $J=7.8 \mathrm{~Hz}), 128.8(2 \mathrm{C}), 128.7,128.4$, $127.7,123.4,122.3,119.9,116.0(\mathrm{~d}, J=21.5 \mathrm{~Hz}), 87.1,85.9,51.5$, 48.0. ${ }^{19} \mathrm{~F}\{1 \mathrm{H}\}$ NMR $\left(376 \mathrm{MHz}, \mathrm{CDCl}_{3}\right) \delta-113.93$. HRMS (ESI) calcd for $\mathrm{C}_{29} \mathrm{H}_{24} \mathrm{FN}_{2} \mathrm{O}(\mathrm{M}+\mathrm{H})^{+} m / z 435.1873$, found $m / z 435.1875$.

1-Benzyl-3-phenyl-1-(3-phenyl-1-(p-tolyl)prop-2-yn-1-yl)urea (3g). Compound $3 \mathrm{~g}$ was synthesized according to the general procedure. ${ }^{25}$ Compound $3 \mathrm{~g}$ was obtained as a light yellow oil. ${ }^{1} \mathrm{H}$ NMR $\left(400 \mathrm{MHz}, \mathrm{CDCl}_{3}\right) \delta 7.68-7.61(\mathrm{~m}, 2 \mathrm{H}), 7.54-7.46(\mathrm{~m}, 6 \mathrm{H})$, 7.45-7.37 (m, 5H), 7.33-7.25 (m, 5H), 7.12-7.05 (m, $2 \mathrm{H}), 6.60$ (bs, $1 \mathrm{H}), 4.78(\mathrm{~d}, J=17.0 \mathrm{~Hz}, 1 \mathrm{H}), 4.59(\mathrm{~d}, J=17.1 \mathrm{~Hz}, 1 \mathrm{H}), 2.49(\mathrm{~s}$, $3 \mathrm{H}) .{ }^{13} \mathrm{C}\{1 \mathrm{H}\} \mathrm{NMR}\left(101 \mathrm{MHz}, \mathrm{CDCl}_{3}\right) \delta 155.4,138.8,138.4,137.9$, $137.2,131.6,129.1,129.0,128.7,128.6,128.5,128.4,128.3,128.0$, 127.1, 124.8, 123.1, 122.4, 119.7, 86.8, 86.2, 51.3, 48.5, 21.5. HRMS (ESI) calcd for $\mathrm{C}_{30} \mathrm{H}_{27} \mathrm{~N}_{2} \mathrm{O}(\mathrm{M}+\mathrm{H})^{+} \mathrm{m} / z$ 431.2123, found $\mathrm{m} / \mathrm{z}$ 431.2121.

1-Benzyl-1-(1-(4-methoxyphenyl)-3-phenylprop-2-yn-1-yl)-3phenylurea (3h). Compound $3 \mathrm{~h}$ was synthesized according to the general procedure. ${ }^{25}$ Compound $3 \mathrm{~h}$ was obtained as a yellow oil. ${ }^{1} \mathrm{H}$ NMR $\left(400 \mathrm{MHz}, \mathrm{CDCl}_{3}\right) \delta 7.72-7.67(\mathrm{~m}, 2 \mathrm{H}), 7.47-7.40(\mathrm{~m}, 6 \mathrm{H})$, 7.39-7.32 (m, 4H), 7.29-7.24 (m, 2H), 7.22-7.18 (m, 2H), 7.07$6.97(\mathrm{~m}, 4 \mathrm{H}), 6.48(\mathrm{bs}, 1 \mathrm{H}), 4.71(\mathrm{~d}, J=17.0 \mathrm{~Hz}, 1 \mathrm{H}), 4.52(\mathrm{~d}, J=$ $17.0 \mathrm{~Hz}, 1 \mathrm{H}), 3.86(\mathrm{~s}, 3 \mathrm{H}) .{ }^{13} \mathrm{C}\{1 \mathrm{H}\} \mathrm{NMR}\left(101 \mathrm{MHz}, \mathrm{CDCl}_{3}\right) \delta$ $159.6,155.6,138.8,137.3,131.7,130.2,129.2,129.1,128.8,128.6$, $128.4,128.1,127.2,123.2,122.5,119.8,114.1,86.8,86.4,55.3,50.8$, 48.5. HRMS (ESI) calcd for $\mathrm{C}_{30} \mathrm{H}_{27} \mathrm{~N}_{2} \mathrm{O}_{2}(\mathrm{M}+\mathrm{H})^{+} \mathrm{m} / z$ 447.2072, found $m / z 447.2075$.

1-Benzyl-1-(1-(3-chlorophenyl)-3-phenylprop-2-yn-1-yl)-3-phenylurea (3i). Compound 3i was synthesized according to the general procedure. ${ }^{25}$ Compound $3 \mathbf{i}$ was obtained as an orange oil. ${ }^{1} \mathrm{H}$ NMR $\left(400 \mathrm{MHz}, \mathrm{CDCl}_{3}\right) \delta 7.82(\mathrm{~s}, 1 \mathrm{H}), 7.72-7.67(\mathrm{~m}, 1 \mathrm{H}), 7.51-7.44$ $(\mathrm{m}, 6 \mathrm{H}), 7.42-7.35(\mathrm{~m}, 6 \mathrm{H}), 7.32-7.23(\mathrm{~m}, 4 \mathrm{H}), 7.13(\mathrm{~s}, 1 \mathrm{H})$, 7.10-7.05 (m, 1H), $6.62(\mathrm{bs}, 1 \mathrm{H}), 4.73(\mathrm{~d}, J=17.2 \mathrm{~Hz}, 1 \mathrm{H}), 4.53(\mathrm{~d}$, $J=17.2 \mathrm{~Hz}, 1 \mathrm{H}) \cdot{ }^{13} \mathrm{C}\{1 \mathrm{H}\}$ NMR $\left(101 \mathrm{MHz}, \mathrm{CDCl}_{3}\right) \delta 155.5,140.2$, $138.5,136.8,134.6,131.6,130.0,129.1,128.8,128.7,128.4,128.3$, $128.1,127.8,126.9,125.9,123.4,122.0,120.0,87.4,85.2,50.8,48.3$. HRMS (ESI) calcd for $\mathrm{C}_{29} \mathrm{H}_{24} \mathrm{ClN}_{2} \mathrm{O}(\mathrm{M}+\mathrm{H})^{+} \mathrm{m} / z$ 451.1577, found $m / z 451.1578$.

1-Benzyl-3-phenyl-1-(3-phenyl-1-(3-vinylphenyl)prop-2-yn-1-yl)urea (3j). Compound $3 \mathbf{j}$ was synthesized according to the general procedure. ${ }^{25}$ Compound $3 \mathbf{j}$ was obtained as an orange oil. ${ }^{1} \mathrm{H}$ NMR $\left(400 \mathrm{MHz}, \mathrm{CDCl}_{3}\right) \delta 7.81(\mathrm{~s}, 1 \mathrm{H}), 7.69(\mathrm{~d}, J=7.4 \mathrm{~Hz}, 1 \mathrm{H}), 7.50-$ $7.35(\mathrm{~m}, 12 \mathrm{H}), 7.30-7.25(\mathrm{~m}, 2 \mathrm{H}), 7.24-7.21(\mathrm{~m}, 2 \mathrm{H}), 7.09$ (brs, $1 \mathrm{H}), 7.08-7.03(\mathrm{~m}, 1 \mathrm{H}), 6.83(\mathrm{dd}, J=17.6,10.9 \mathrm{~Hz}, 1 \mathrm{H}), 6.53(\mathrm{bs}$, $1 \mathrm{H}), 5.87(\mathrm{~d}, J=17.6 \mathrm{~Hz}, 1 \mathrm{H}), 5.36(\mathrm{~d}, J=11.2 \mathrm{~Hz}, 1 \mathrm{H}), 4.73(\mathrm{~d}, J=$ $17.0 \mathrm{~Hz}, 1 \mathrm{H}), 4.54(\mathrm{~d}, J=17.1 \mathrm{~Hz}, 1 \mathrm{H}) .{ }^{13} \mathrm{C}\{1 \mathrm{H}\} \mathrm{NMR}(101 \mathrm{MHz}$, $\left.\mathrm{CDCl}_{3}\right) \delta 155.6,138.7,138.4,138.1,137.1,136.5,131.7,129.2,129.0$, 
$128.8,128.6,128.4,128.1,127.2,127.1,126.0,125.8,123.3,122.4$, $119.8,114.6,87.1,85.9,51.3$, 48.6. HRMS (ESI) calcd for $\mathrm{C}_{31} \mathrm{H}_{27} \mathrm{~N}_{2} \mathrm{O}(\mathrm{M}+\mathrm{H})^{+} m / z$ 443.2123, found $m / z$ 443.2127.

1-Benzyl-1-(1-(2-bromophenyl)-3-phenylprop-2-yn-1-yl)-3-phenylurea ( $3 k$ ). Compound $3 \mathbf{k}$ was synthesized according to the general procedure. ${ }^{25}$ Compound $3 \mathbf{k}$ was obtained as a yellow solid; $\mathrm{mp}$ (hexane) $124.7-126.1{ }^{\circ} \mathrm{C} .{ }^{1} \mathrm{H}$ NMR $\left(400 \mathrm{MHz}, \mathrm{CDCl}_{3}\right) \delta 7.97(\mathrm{dd}, J$ $=7.7,1.6 \mathrm{~Hz}, 1 \mathrm{H}), 7.68(\mathrm{dd}, J=7.9,1.2 \mathrm{~Hz}, 1 \mathrm{H}), 7.55-7.51(\mathrm{~m}, 2 \mathrm{H})$, 7.41-7.34 (m, 6H), 7.33-7.24 (m, 8H), $7.09(\mathrm{~s}, 1 \mathrm{H}), 7.06-7.01(\mathrm{~m}$, $1 \mathrm{H}), 6.59(\mathrm{bs}, 1 \mathrm{H}), 4.62(\mathrm{~s}, 2 \mathrm{H}) .{ }^{13} \mathrm{C}\{1 \mathrm{H}\} \mathrm{NMR}\left(101 \mathrm{MHz}, \mathrm{CDCl}_{3}\right)$ $\delta$ 155.5, 138.8, 137.2, 136.6, 133.6, 131.8, 130.8, 130.1, 129.1, 128.8, $128.7,128.4,127.9,127.6,126.9,124.6,123.1,122.3,119.7,87.0$, 85.8, 52.2, 48.3. HRMS (ESI) calcd for $\mathrm{C}_{29} \mathrm{H}_{24} \mathrm{BrN}_{2} \mathrm{O}(\mathrm{M}+\mathrm{H})^{+} \mathrm{m} / z$ 495.1072, found $\mathrm{m} / \mathrm{z}$ 495.1074.

1-Benzyl-1-(1,3-diphenylprop-2-yn-1-yl)-3-propylurea (3I). Compound 31 was synthesized according to the general procedure. ${ }^{25}$ Compound 31 was isolated as a yellow oil in $45 \%$ yield $(172 \mathrm{mg}) .{ }^{1} \mathrm{H}$ NMR $\left(300 \mathrm{MHz}, \mathrm{CDCl}_{3}\right) \delta 7.70-7.64(\mathrm{~m}, 2 \mathrm{H}), 7.44-7.24(\mathrm{~m}$, $13 \mathrm{H}), 6.98(\mathrm{bs}, 1 \mathrm{H}), 4.54(\mathrm{~d}, J=17.1 \mathrm{~Hz}, 1 \mathrm{H}), 4.41-4.34(\mathrm{~m}, 1 \mathrm{H})$, $4.31(\mathrm{~d}, J=17.1 \mathrm{~Hz}, 1 \mathrm{H}), 3.26-3.04(\mathrm{~m}, 2 \mathrm{H}), 1.40-1.25(\mathrm{~m}, 2 \mathrm{H})$, $0.69(\mathrm{t}, J=7.4 \mathrm{~Hz}, 3 \mathrm{H}) .{ }^{13} \mathrm{C}\{1 \mathrm{H}\} \operatorname{NMR}\left(75 \mathrm{MHz}, \mathrm{CDCl}_{3}\right) \delta 158.2$, $138.6,137.9,131.7,128.9,128.7,128.5,128.4,128.1,127.8,127.7$, $126.9,122.7,86.7,86.56,51.4,48.3,42.8,23.2,11.1$. HRMS (ESI) calcd for $\mathrm{C}_{26} \mathrm{H}_{27} \mathrm{~N}_{2} \mathrm{O}(\mathrm{M}+\mathrm{H})^{+} \mathrm{m} / z$ 383.2123, found $m / z$ 383.2126.

1,3-Dibenzyl-1-(1,3-diphenylprop-2-yn-1-yl)urea (3m). Compound $3 \mathrm{~m}$ was synthesized according to the general procedure. ${ }^{25}$ Compound $3 \mathrm{~m}$ was isolated as a yellow oil in $62 \%$ yield $(267 \mathrm{mg}) .{ }^{1} \mathrm{H}$ NMR $\left(300 \mathrm{MHz}, \mathrm{CDCl}_{3}\right) \delta$ 7.77-7.70 (m, 2H), 7.48-7.30 (m, $12 \mathrm{H}), 7.28-7.22(\mathrm{~m}, 3 \mathrm{H}), 7.06-7.00(\mathrm{~m}, 3 \mathrm{H}), 4.84(\mathrm{t}, J=5.5 \mathrm{~Hz}$, $1 \mathrm{H}), 4.63(\mathrm{~d}, J=17.2 \mathrm{~Hz}, 1 \mathrm{H}), 4.54-4.35(\mathrm{~m}, 3 \mathrm{H}) .{ }^{13} \mathrm{C}\{1 \mathrm{H}\} \mathrm{NMR}$ $\left(75 \mathrm{MHz}, \mathrm{CDCl}_{3}\right) \delta 157.9,139.1,138.4,137.6,131.7,128.9,128.7$, $128.5,128.4,128.3,128.1,127.7,127.6,127.1,127.0,126.9,122.6$, 86.8, 86.3, 51.5, 48.3, 44.9. HRMS (ESI) calcd for $\mathrm{C}_{30} \mathrm{H}_{27} \mathrm{~N}_{2} \mathrm{O}(\mathrm{M}+$ $\mathrm{H})^{+} \mathrm{m} / z$ 431.2123, found $\mathrm{m} / z$ 431.2121.

1-Benzyl-3-phenyl-1-(1-phenyl-3-(p-tolyl)prop-2-yn-1-yl)urea (3n). Compound $3 n$ was synthesized according to the general procedure. $^{25}$ Compound $3 \mathbf{n}$ was obtained as a yellowish solid; $\mathrm{mp}$ (hexane) 53.6-54.2 ${ }^{\circ} \mathrm{C} .{ }^{1} \mathrm{H}$ NMR $\left(400 \mathrm{MHz}, \mathrm{CDCl}_{3}\right) \delta 7.79-7.72$ (m, $2 \mathrm{H}), 7.49-7.32(\mathrm{~m}, 10 \mathrm{H}), 7.28-7.23(\mathrm{~m}, 2 \mathrm{H}), 7.21-7.13(\mathrm{~m}$, $4 \mathrm{H}), 7.06-6.99(\mathrm{~m}, 2 \mathrm{H}), 6.45(\mathrm{bs}, 1 \mathrm{H}), 4.71(\mathrm{~d}, J=17.0 \mathrm{~Hz}, 1 \mathrm{H})$, $4.50(\mathrm{~d}, J=17.0 \mathrm{~Hz}, 1 \mathrm{H}), 2.40(\mathrm{~s}, 3 \mathrm{H}) .{ }^{13} \mathrm{C}\{1 \mathrm{H}\}$ NMR $(101 \mathrm{MHz}$, $\mathrm{CDCl}_{3}$ ) $\delta 155.6,138.9,138.8,138.3,137.3,131.7,129.3,129.2,128.9$, 128.8, 128.3, 128.2, 127.8, 127.2, 123.2, 119.8, 119.4, 87.2, 85.4, 51.5, 48.7, 21.6. HRMS (ESI) calcd for $\mathrm{C}_{30} \mathrm{H}_{27} \mathrm{~N}_{2} \mathrm{O}(\mathrm{M}+\mathrm{H})^{+} \mathrm{m} / z$ 431.2123, found $\mathrm{m} / z$ 431.2124.

1-Benzyl-1-(3-(4-chlorophenyl)-1-phenylprop-2-yn-1-yl)-3-phenylurea (30). Compound 30 was synthesized according to the general procedure. ${ }^{25}$ Compound 30 was obtained as a yellow viscous oil. ${ }^{1} \mathrm{H}$ NMR $\left(400 \mathrm{MHz}, \mathrm{CDCl}_{3}\right) \delta 7.74(\mathrm{~d}, J=7.5 \mathrm{~Hz}, 2 \mathrm{H}), 7.50-7.29(\mathrm{~m}$, $12 \mathrm{H}), 7.28-7.22(\mathrm{~m}, 2 \mathrm{H}), 7.21-7.15(\mathrm{~m}, 2 \mathrm{H}), 7.10-7.00(\mathrm{~m}, 2 \mathrm{H})$, $6.44(\mathrm{bs}, 1 \mathrm{H}), 4.68(\mathrm{~d}, J=17.1 \mathrm{~Hz}, 1 \mathrm{H}), 4.51(\mathrm{~d}, J=17.1 \mathrm{~Hz}, 1 \mathrm{H})$. ${ }^{13} \mathrm{C}\{1 \mathrm{H}\}$ NMR $\left(101 \mathrm{MHz}, \mathrm{CDCl}_{3}\right) \delta 155.6,138.8,137.9,137.2$, $134.7,133.0,129.3,128.9,128.8,128.4,128.2,127.8,127.1,123.3$, 121.0, 119.9, 87.2, 85.9, 51.4, 48.6. HRMS (ESI) calcd for $\mathrm{C}_{29} \mathrm{H}_{24} \mathrm{ClN}_{2} \mathrm{O}(\mathrm{M}+\mathrm{H})^{+} \mathrm{m} / z$ 451.1577, found $\mathrm{m} / z$ 451.1579.

1-Benzyl-1-(3-(4-fluorophenyl)-1-phenylprop-2-yn-1-yl)-3-phenylurea (3p). Compound $3 p$ was synthesized according to the general procedure. ${ }^{25}$ Compound $3 \mathrm{p}$ was obtained as a viscous yellow oil. ${ }^{1} \mathrm{H}$ NMR $\left(400 \mathrm{MHz}, \mathrm{CDCl}_{3}\right) \delta 7.81(\mathrm{~d}, J=7.5 \mathrm{~Hz}, 2 \mathrm{H}), 7.56-7.35$ (m, $10 \mathrm{H}), 7.33-7.22(\mathrm{~m}, 4 \mathrm{H}), 7.13(\mathrm{~s}, 1 \mathrm{H}), 7.10-7.00(\mathrm{~m}, 3 \mathrm{H}), 6.60(\mathrm{bs}$, $1 \mathrm{H}), 4.74(\mathrm{~d}, J=17.1 \mathrm{~Hz}, 1 \mathrm{H}), 4.58(\mathrm{~d}, J=17.1 \mathrm{~Hz}, 1 \mathrm{H}) .{ }^{13} \mathrm{C}\{1 \mathrm{H}\}$ $\mathrm{NMR}\left(101 \mathrm{MHz}, \mathrm{CDCl}_{3}\right) \delta 162.6\left(\mathrm{~d}, J_{\mathrm{C}, \mathrm{F}}=249.9 \mathrm{~Hz}\right), 155.5,138.8$, $137.9,137.2,133.6\left(\mathrm{~d}, J_{\mathrm{C}, \mathrm{F}}=8.4 \mathrm{~Hz}\right), 129.1,128.7(2 \mathrm{C}), 128.2,127.9$, $127.7,127.0,123.2,119.8,118.5\left(\mathrm{~d}, J_{\mathrm{C}, \mathrm{F}}=3.5 \mathrm{~Hz}\right), 115.6\left(\mathrm{~d}, J_{\mathrm{C}, \mathrm{F}}=\right.$ $22.1 \mathrm{~Hz}), 85.8(2 \mathrm{C}), 51.2,48.4 .{ }^{19} \mathrm{~F}\{1 \mathrm{H}\} \operatorname{NMR}\left(376 \mathrm{MHz} \mathrm{CDCl}_{3}\right) \delta$ -110.00. HRMS (ESI) calcd for $\mathrm{C}_{29} \mathrm{H}_{24} \mathrm{FN}_{2} \mathrm{O}(\mathrm{M}+\mathrm{H})^{+} \mathrm{m} / z$ 435.1873, found $m / z 435.1872$.

4,4-Dimethyl-5-methylene-1-phenylimidazolidin-2-one (2a). Compound $2 \mathrm{a}$ was obtained from $1 \mathrm{a}$ in $1 \mathrm{~h}$, according to the general procedure. The crude was purified by flash column chromatography using hexane/ethyl acetate $(1: 1)$ as eluent to give 2 a $(78 \mathrm{mg}, 97 \%$ yield) as a white solid; mp (hexane) $127.5-130.1{ }^{\circ} \mathrm{C} .{ }^{1} \mathrm{H}$ NMR (400 $\left.\mathrm{MHz}_{\mathrm{CDCl}}\right) \delta 7.48-7.42(\mathrm{~m}, 2 \mathrm{H}), 7.35-7.30(\mathrm{~m}, 3 \mathrm{H}), 6.23(\mathrm{bs}$, $1 \mathrm{H}), 4.03,3.97$ (2 partly overlapping bs, $2 \mathrm{H}), 1.46(\mathrm{~s}, 6 \mathrm{H}) .{ }^{13} \mathrm{C}\{1 \mathrm{H}\}$ NMR $\left(101 \mathrm{MHz}, \mathrm{CDCl}_{3}\right) \delta 157.2,154.68,135.2,129.4,127.7,127.6$, 79.6, 56.7, 29.9. HRMS (ESI) calcd for $\mathrm{C}_{12} \mathrm{H}_{15} \mathrm{~N}_{2} \mathrm{O}(\mathrm{M}+\mathrm{H})^{+} \mathrm{m} / z$ 203.1184, found $\mathrm{m} / z 203.1182$.

4,4-Dimethyl-5-methylene-1-(p-tolyl)imidazolidin-2-one (2b). Compound $\mathbf{2 b}$ was obtained from $\mathbf{1 b}$ in $16 \mathrm{~h}$, according to the general procedure. The crude was purified by flash column chromatography using hexane/ethyl acetate (1:1) as eluent to give 2b (69 mg, 82\% yield) as a pale orange solid; mp (hexane) 195.2$197.3{ }^{\circ} \mathrm{C} .{ }^{1} \mathrm{H}$ NMR $\left(400 \mathrm{MHz}, \mathrm{CDCl}_{3}\right) \delta 7.27(\mathrm{~d}, J=8.0 \mathrm{~Hz}, 2 \mathrm{H})$, $7.21(\mathrm{~d}, J=8.3 \mathrm{~Hz}, 2 \mathrm{H}), 5.83(\mathrm{bs}, 1 \mathrm{H}), 4.02(\mathrm{~d}, J=2.1 \mathrm{~Hz}, 1 \mathrm{H}), 3.97$ $(\mathrm{d}, J=2.3 \mathrm{~Hz}, 1 \mathrm{H}), 2.39(\mathrm{~s}, 3 \mathrm{H}), 1.48(\mathrm{~s}, 6 \mathrm{H}) .{ }^{13} \mathrm{C}\{1 \mathrm{H}\} \mathrm{NMR}(101$ $\left.\mathrm{MHz}, \mathrm{CDCl}_{3}\right) \delta 157.3,154.9,137.6,132.5,130.1,127.6,79.5,56.7$, 29.9, 21.3. HRMS (ESI) calcd for $\mathrm{C}_{13} \mathrm{H}_{17} \mathrm{~N}_{2} \mathrm{O}(\mathrm{M}+\mathrm{H})^{+} \mathrm{m} / z$ 217.1341, found $m / z 217.1344$.

1-(4-Fluorophenyl)-4,4-dimethyl-5-methyleneimidazolidin-2one (2c). Compound $2 \mathrm{c}$ was obtained from $1 \mathrm{c}$ in $1 \mathrm{~h}$ and $30 \mathrm{~min}$, according to the general procedure. The crude was purified by flash column chromatography using hexane/ethyl acetate (1:1) as eluent to give $2 \mathrm{c}(86 \mathrm{mg}$, 97\% yield) as an orange solid; $\mathrm{mp}$ (hexane) $165.6-$ $167.0^{\circ} \mathrm{C} .{ }^{1} \mathrm{H}$ NMR $\left(400 \mathrm{MHz}, \mathrm{CDCl}_{3}\right) \delta 7.33-7.25(\mathrm{~m}, 2 \mathrm{H}), 7.17-$ $7.08(\mathrm{~m}, 2 \mathrm{H}), 5.89(\mathrm{bs}, 1 \mathrm{H}), 4.03,3.97(2 \mathrm{bs}, 2 \mathrm{H}), 1.46(\mathrm{~s}, 6 \mathrm{H})$. ${ }^{13} \mathrm{C}\{1 \mathrm{H}\} \mathrm{NMR}\left(101 \mathrm{MHz}, \mathrm{CDCl}_{3}\right) \delta 161.7\left(\mathrm{~d}, J_{\mathrm{C}, \mathrm{F}}=247.1 \mathrm{~Hz}\right)$, 157.1, 154.7, $131.1\left(\mathrm{~d}, J_{\mathrm{C}, \mathrm{F}}=3.1 \mathrm{~Hz}\right), 129.6\left(\mathrm{~d}, J_{\mathrm{C}, \mathrm{F}}=8.6 \mathrm{~Hz}\right), 116.4$ $\left(\mathrm{d}, J_{\mathrm{C}, \mathrm{F}}=22.8 \mathrm{~Hz}\right), 79.9,56.8,29.9 .{ }^{19} \mathrm{~F}\{1 \mathrm{H}\}$ NMR $(376 \mathrm{MHz}$, $\left.\mathrm{CDCl}_{3}\right) \delta-113.93$. HRMS (ESI) calcd for $\mathrm{C}_{12} \mathrm{H}_{14} \mathrm{FN}_{2} \mathrm{O}(\mathrm{M}+\mathrm{H})^{+}$ $\mathrm{m} / z 221.1090$, found $\mathrm{m} / z 221.1086$.

Methyl 4-(4,4-Dimethyl-5-methylene-2-oxoimidazolidin-1-yl)benzoate (2d). Compound $2 \mathrm{~d}$ was obtained from $1 \mathrm{~d}$ in $6 \mathrm{~h}$, according to the general procedure. The crude was purified by flash column chromatography using hexane/ethyl acetate (1:1) as eluent to give $2 \mathrm{~d}$ (102 $\mathrm{mg}, 98 \%$ yield) as a yellowish solid; mp (hexane) $218.0-220.3^{\circ} \mathrm{C} .{ }^{1} \mathrm{H}$ NMR $\left(400 \mathrm{MHz}, \mathrm{CDCl}_{3}\right) \delta 8.14-8.07(\mathrm{~m}, 2 \mathrm{H})$, $7.48-7.40(\mathrm{~m}, 2 \mathrm{H}), 6.34$ (bs, $1 \mathrm{H}), 4.17(\mathrm{dd}, J=2.6,0.8 \mathrm{~Hz}, 1 \mathrm{H})$, $4.04(\mathrm{~d}, J=2.6 \mathrm{~Hz}, 1 \mathrm{H}), 3.91(\mathrm{~s}, 3 \mathrm{H}), 1.45(\mathrm{~s}, 6 \mathrm{H}) .{ }^{13} \mathrm{C}\{1 \mathrm{H}\} \mathrm{NMR}$ $\left(101 \mathrm{MHz}, \mathrm{CDCl}_{3}\right) \delta 166.5,156.6,153.5,139.6,130.7,128.6,127.0$, 80.6, 56.8, 52.3, 29.8. HRMS (ESI) calcd for $\mathrm{C}_{14} \mathrm{H}_{17} \mathrm{~N}_{2} \mathrm{O}_{3}(\mathrm{M}+\mathrm{H})^{+}$ $\mathrm{m} / z$ 261.1240, found $\mathrm{m} / z 261.1242$.

4,4-Diethyl-5-methylene-1-phenylimidazolidin-2-one (2e). Compound $2 \mathrm{e}$ was obtained from $1 \mathrm{e}$ in $3 \mathrm{~h}$, according to the general procedure. The crude was purified by flash column chromatography using hexane/ethyl acetate (1:1) as eluent to give 2 e $(92 \mathrm{mg}$, $99 \%$ yield) as a pale orange solid; mp (hexane) $120.5-122.2{ }^{\circ} \mathrm{C} .{ }^{1} \mathrm{H}$ NMR $\left(400 \mathrm{MHz}, \mathrm{CDCl}_{3}\right) \delta 7.48-7.42(\mathrm{~m}, 2 \mathrm{H}), 7.36-7.31(\mathrm{~m}, 1 \mathrm{H}), 7.30-$ $7.27(\mathrm{~m}, 2 \mathrm{H}), 5.32(\mathrm{bs}, 1 \mathrm{H}), 4.13(\mathrm{dd}, J=2.3,0.8 \mathrm{~Hz}, 1 \mathrm{H}), 3.87(\mathrm{~d}, J$ $=2.3 \mathrm{~Hz}, 1 \mathrm{H}), 1.75-1.57(\mathrm{~m}, 4 \mathrm{H}), 0.97(\mathrm{t}, J=7.3 \mathrm{~Hz}, 6 \mathrm{H}) .{ }^{13} \mathrm{C}\{1 \mathrm{H}\}$ NMR $\left(101 \mathrm{MHz}, \mathrm{CDCl}_{3}\right) \delta 157.9,150.9,135.2,129.5,127.8,127.7$, 80.2, 63.6, 34.1, 7.8. HRMS (ESI) calcd for $\mathrm{C}_{14} \mathrm{H}_{19} \mathrm{~N}_{2} \mathrm{O}(\mathrm{M}+\mathrm{H})^{+} \mathrm{m} /$ $z$ 231.1497, found $\mathrm{m} / \mathrm{z} 231.1499$.

4-Methylene-3-phenyl-1,3-diazaspiro[4.5]decan-2-one (2f). Compound $2 \mathrm{f}$ was obtained from $1 \mathrm{f}$ in $16 \mathrm{~h}$, according to the general procedure. The crude was purified by flash column chromatography using hexane/ethyl acetate $(1: 1)$ as eluent to give 2f (92 mg, 95\% yield) as a white solid; mp (hexane) $175.2-176.7^{\circ} \mathrm{C}$. ${ }^{1} \mathrm{H}$ NMR (400 MHz, $\mathrm{CDCl}_{3}$ ) $\delta$ 7.47-7.41 (m, 2H), 7.34-7.29 (m, $3 \mathrm{H}), 6.07(\mathrm{bs}, 1 \mathrm{H}), 4.07(\mathrm{~d}, J=2.3 \mathrm{~Hz}, 1 \mathrm{H}), 3.96(\mathrm{~d}, J=2.3 \mathrm{~Hz}, 1 \mathrm{H})$, $1.92-1.83(\mathrm{~m}, 2 \mathrm{H}), 1.81-1.70(\mathrm{~m}, 2 \mathrm{H}), 1.63-1.53(\mathrm{~m}, 2 \mathrm{H}), 1.49-$ $1.35(\mathrm{~m}, 2 \mathrm{H}), 1.35-1.21(\mathrm{~m}, 2 \mathrm{H}) .{ }^{13} \mathrm{C}\{1 \mathrm{H}\}$ NMR $(101 \mathrm{MHz}$, $\left.\mathrm{CDCl}_{3}\right) \delta 157.2,154.5,135.1,129.4,127.7,127.6,80.2,59.5,38.6$, 25.0, 22.4. HRMS (ESI) calcd for $\mathrm{C}_{15} \mathrm{H}_{19} \mathrm{~N}_{2} \mathrm{O}(\mathrm{M}+\mathrm{H})^{+} \mathrm{m} / z$ 243.1497, found $m / z 243.1500$.

3-Benzyl-4,4-dimethyl-5-methylene-1-phenylimidazolidin-2-one (2g). Compound $2 \mathrm{~g}$ was obtained from $1 \mathrm{~g}$ in $16 \mathrm{~h}$, according to the general procedure. The crude was purified by flash column chromatography using hexane/ethyl acetate (7:3) as eluent to give $2 \mathrm{~g}\left(61 \mathrm{mg}, 52 \%\right.$ yield) as a colorless oil. ${ }^{1} \mathrm{H} \mathrm{NMR}\left(400 \mathrm{MHz}, \mathrm{CDCl}_{3}\right)$ $\delta 7.47(\mathrm{~d}, J=7.2 \mathrm{~Hz}, 2 \mathrm{H}), 7.39-7.25(\mathrm{~m}, 5 \mathrm{H}), 7.20-7.16(\mathrm{~m}, 2 \mathrm{H})$, 
$7.06-7.00(\mathrm{~m}, 1 \mathrm{H}), 4.64(\mathrm{~d}, J=3.3 \mathrm{~Hz}, 1 \mathrm{H}), 4.63(\mathrm{~s}, 2 \mathrm{H}), 4.20(\mathrm{~d}, J$ $=3.3 \mathrm{~Hz}, 1 \mathrm{H}), 1.36(\mathrm{~s}, 6 \mathrm{H}) \cdot{ }^{13} \mathrm{C}\{1 \mathrm{H}\} \operatorname{NMR}\left(101 \mathrm{MHz}, \mathrm{CDCl}_{3}\right) \delta$ $162.8,149.2,147.0,139.0,128.6,128.5,127.8,127.3,123.8,122.3$, 83.3, 61.1, 44.4, 27.6. HRMS (ESI) calcd for $\mathrm{C}_{19} \mathrm{H}_{21} \mathrm{~N}_{2} \mathrm{O}(\mathrm{M}+\mathrm{H})^{+}$ $\mathrm{m} / z$ 293.1654, found $\mathrm{m} / z 293.1652$.

3-Benzyl-4,4-dimethyl-5-nonylidene-1-phenylimidazolidin-2one (2h). Compound $2 \mathrm{~h}$ was obtained from $1 \mathrm{~h}$ in $16 \mathrm{~h}$, according to the general procedure. The crude was purified by flash column chromatography using hexane/ethyl acetate (7:3) as eluent to give a mixture of $E$ and $Z(150 \mathrm{mg}, 83 \%)$ in a ca. 1:0.06 molar ratio. The major $Z$ isomer was characterized as follows: ${ }^{1} \mathrm{H}$ NMR $(400 \mathrm{MHz}$, $\left.\mathrm{CDCl}_{3}\right) \delta 7.45-7.40(\mathrm{~m}, 4 \mathrm{H}), 7.38-7.30(\mathrm{~m}, 5 \mathrm{H}), 7.30-7.26(\mathrm{~m}$, $1 \mathrm{H}), 4.54(\mathrm{~s}, 2 \mathrm{H}), 4.34(\mathrm{t}, J=7.3 \mathrm{~Hz}, 1 \mathrm{H}), 1.47-1.41(\mathrm{~m}, 2 \mathrm{H}), 1.35$ $(\mathrm{s}, 6 \mathrm{H}), 1.32-1.27(\mathrm{~m}, 2 \mathrm{H}), 1.25-1.04(\mathrm{~m}, 10 \mathrm{H}), 0.91(\mathrm{t}, J=7.1 \mathrm{~Hz}$, $3 \mathrm{H}) .{ }^{13} \mathrm{C}\{1 \mathrm{H}\} \mathrm{NMR}\left(101 \mathrm{MHz}, \mathrm{CDCl}_{3}\right) \delta 157.4,143.3,139.6,138.1$, $128.7,128.5,127.9,127.7,127.2,126.9,99.4,61.1,43.4,31.9,30.0$, 29.3, 29.2, 29.0, 27.9, 26.1, 22.7, 14.1.HRMS (ESI) calcd for $\mathrm{C}_{27} \mathrm{H}_{37} \mathrm{~N}_{2} \mathrm{O}(\mathrm{M}+\mathrm{H})^{+} m / z$ 405.2906, found $m / z$ 405.2904.

5-Benzylidene-4,4-dimethyl-1-phenylimidazolidin-2-one (2i). Compound $2 \mathbf{i}$ was obtained from $\mathbf{1 i}$ in $16 \mathrm{~h}$, according to the general procedure. The crude was purified by flash column chromatography using hexane/ethyl acetate $(1: 1)$ as eluent to give two fractions, one containing the pure $2 \mathrm{i} Z$ isomer $(62 \mathrm{mg})$, the other containing a mixture of $E$ and $Z\left(35 \mathrm{mg}\right.$; see ${ }^{1} \mathrm{H}$ NMR spectrum of the mixture in the SI). The overall yield of $Z$ and $E$ isomers of $2 \mathbf{i}$ amounted to $87 \%(97 \mathrm{mg})$ with $Z / E$ isomers in a 1:0.25 molar ratio. The major isomer $Z$ was isolated as a yellowish solid ( $\mathrm{mp}$ from hexane: $\left.216.5-217.8^{\circ} \mathrm{C}\right)$ and characterized as follows: ${ }^{1} \mathrm{H}$ NMR $(400$ $\left.\mathrm{MHz}, \mathrm{CDCl}_{3}\right) \delta 7.06-6.95(\mathrm{~m}, 5 \mathrm{H}), 6.88-6.80(\mathrm{~m}, 3 \mathrm{H}), 6.68(\mathrm{dd}, J$ $=7.2,1.9 \mathrm{~Hz}, 2 \mathrm{H}), 5.81(\mathrm{bs}, 1 \mathrm{H}), 5.59(\mathrm{~s}, 1 \mathrm{H}), 1.57(\mathrm{~s}, 6 \mathrm{H})$. ${ }^{13} \mathrm{C}\{1 \mathrm{H}\}$ NMR $\left(101 \mathrm{MHz}, \mathrm{CDCl}_{3}\right) \delta 157.8,145.0,135.9,134.6$, 128.3, 128.1, 127.0, 126.2, 126.1, 125.2, 99.0, 57.6, 30.1. HRMS (ESI) calcd for $\mathrm{C}_{18} \mathrm{H}_{19} \mathrm{~N}_{2} \mathrm{O}(\mathrm{M}+\mathrm{H})^{+} \mathrm{m} / z$ 279.1497, found $\mathrm{m} / z 279.1498$.

4,4-Dimethyl-1-phenyl-5-(4-(trifluoromethyl)benzylidene)imidazolidin-2-one (2j). Compound $2 \mathbf{j}$ was obtained from $1 \mathbf{j}$ in $4 \mathrm{~h}$, according to the general procedure. The crude was purified by flash column chromatography using hexane/ethyl acetate $(1: 1)$ as eluent to give two fractions, one containing the pure $2 \mathrm{j} Z$ isomer $(85 \mathrm{mg})$, the other containing a mixture of $E$ and $Z\left(43 \mathrm{mg}\right.$; see ${ }^{1} \mathrm{H}$ NMR spectrum of the mixture in the SI). The overall yield of $Z$ and $E$ isomers of $2 j$ amounted to $93 \%(128 \mathrm{mg}$ ) with $Z / E$ isomers in a 1:0.3 molar ratio. The major isomer $Z$ was isolated as a white solid ( $\mathrm{mp}$ from hexane: 208.5-211.4 $\left.{ }^{\circ} \mathrm{C}\right)$ and characterized as follows: ${ }^{1} \mathrm{H}$ NMR $(400 \mathrm{MHz}$, $\left.\mathrm{CDCl}_{3}\right) \delta 7.54-7.47(\mathrm{~m}, 4 \mathrm{H}), 7.41-7.34(\mathrm{~m}, 3 \mathrm{H}), 7.29(\mathrm{~d}, J=8.6$ $\mathrm{Hz}, 2 \mathrm{H}), 5.61$ (s, 1H), 5.49 (bs, $1 \mathrm{H}), 1.42(\mathrm{~s}, 6 \mathrm{H}) .{ }^{13} \mathrm{C}\{1 \mathrm{H}\}$ NMR $\left(101 \mathrm{MHz}, \mathrm{CDCl}_{3}\right) \delta 156.7,149.5,140.1\left(\mathrm{q}, J_{\mathrm{C}, \mathrm{F}}=0.9 \mathrm{~Hz}\right), 134.9$, $130.3,129.8,128.6\left(\mathrm{q}, J_{\mathrm{C}, \mathrm{F}}=29.5 \mathrm{~Hz}\right), 128.5,128.3,125.0\left(\mathrm{q}, J_{\mathrm{C}, \mathrm{F}}=\right.$ $3.8 \mathrm{~Hz}), 124.3\left(\mathrm{q}, J_{\mathrm{C}, \mathrm{F}}=271.8 \mathrm{~Hz}\right), 99.9,57.8,29.2 .{ }^{19} \mathrm{~F}\{1 \mathrm{H}\} \mathrm{NMR}$ $\left(376 \mathrm{MHz}, \mathrm{CDCl}_{3}\right) \delta-62.44$. HRMS (ESI) calcd for $\mathrm{C}_{19} \mathrm{H}_{18} \mathrm{~F}_{3} \mathrm{~N}_{2} \mathrm{O}$ $(\mathrm{M}+\mathrm{H})^{+} \mathrm{m} / z$ 347.1371, found $m / z$ 347.1369.

5-(4-Methoxybenzylidene)-4,4-dimethyl-1-phenylimidazolidin2 -one ( $2 k$ ). Compound $2 \mathrm{k}$ was obtained from $1 \mathrm{k}$ in $16 \mathrm{~h}$, according to the general procedure. The crude was purified by flash column chromatography using hexane/ethyl acetate $(1: 1)$ as eluent to give two fractions, one containing the pure $2 \mathrm{k} Z$ isomer $(32 \mathrm{mg})$, the other containing a mixture of $E$ and $Z\left(71 \mathrm{mg}\right.$; see ${ }^{1} \mathrm{H}$ NMR spectrum of the mixture in the SI). The overall yield of $Z$ and $E$ isomers of $\mathbf{2 k}$ amounted to $84 \%(103 \mathrm{mg})$ with $Z / E$ isomers in a 1:0.04 molar ratio. The major isomer $Z$ was isolated as a white solid ( $\mathrm{mp}$ from hexane: 182.4-184.7 ${ }^{\circ} \mathrm{C}$ ) and characterized as follows: ${ }^{1} \mathrm{H}$ NMR $(400 \mathrm{MHz}$, $\left.\mathrm{CDCl}_{3}\right) \delta 7.08-6.96(\mathrm{~m}, 5 \mathrm{H}), 6.59(\mathrm{~d}, J=8.7 \mathrm{~Hz}, 2 \mathrm{H}), 6.39(\mathrm{~d}, J=$ $8.7 \mathrm{~Hz}, 2 \mathrm{H}), 5.92(\mathrm{bs}, 1 \mathrm{H}), 5.54(\mathrm{~s}, 1 \mathrm{H}), 3.65(\mathrm{~s}, 3 \mathrm{H}), 1.55(\mathrm{~s}, 6 \mathrm{H})$. ${ }^{13} \mathrm{C}\{1 \mathrm{H}\}$ NMR $\left(101 \mathrm{MHz}, \mathrm{CDCl}_{3}\right) \delta 157.9,157.3,143.9,135.9$, 129.3, 128.0, 127.2, 126.1, 126.0, 112.6, 98.7, 57.5, 55.3, 30.1. HRMS (ESI) calcd for $\mathrm{C}_{19} \mathrm{H}_{21} \mathrm{~N}_{2} \mathrm{O}_{2}(\mathrm{M}+\mathrm{H})^{+} \mathrm{m} / z$ 309.1603, found $m / z$ 309.1607.

4,4-Dimethyl-1-phenyl-5-(thiophen-2-ylmethylene)imidazolidin2-one (2l). Compound 21 was obtained from 11 in $1 \mathrm{~h}$ and $30 \mathrm{~min}$, according to the general procedure. The crude was purified by flash column chromatography using hexane/ethyl acetate (1:1) as eluent to give a mixture of $E$ and $Z(110 \mathrm{mg}, 97 \%)$ in a 1:0.8 molar ratio. $E$ and $Z$ isomers were characterized as follows: ${ }^{1} \mathrm{H}$ NMR $(400 \mathrm{MHz}$, $\left.\mathrm{CDCl}_{3}\right) \delta 7.51-7.46(\mathrm{~m}, 1 \mathrm{H}(Z)+1 \mathrm{H}(E)), 7.43-7.31(\mathrm{~m}, 3 \mathrm{H})$, 7.19-7.06 (m, 6H), 6.96-6.85 (m, 1H(Z) + 1H(E)), $6.77(\mathrm{~d}, J=3.4$ $\mathrm{Hz}, 1 \mathrm{H}), 6.48$ (dd, $J=5.0,3.7 \mathrm{~Hz}, 1 \mathrm{H}), 6.13(\mathrm{bs}, 1 \mathrm{H}), 6.01(\mathrm{bs}, 1 \mathrm{H})$, $5.98(\mathrm{~d}, J=3.5 \mathrm{~Hz}, 1 \mathrm{H}), 5.57(\mathrm{~s}, 1 \mathrm{H}(E)), 5.51(\mathrm{~s}, 1 \mathrm{H}(\mathrm{Z})), 1.57(\mathrm{~s}$, $6 \mathrm{H}(Z)), 1.55(\mathrm{~s}, 6 \mathrm{H}(E)) .{ }^{13} \mathrm{C}\{1 \mathrm{H}\} \mathrm{NMR}\left(101 \mathrm{MHz}, \mathrm{CDCl}_{3}\right) \delta$ $157.6,156.8,150.1,145.6,137.5,136.8,135.7,134.9,129.7,128.7$, $128.2,127.3,126.8,126.6,126.5,126.5,126.3,124.8,123.8,93.1$, 91.5, 57.9, 57.7, 29.9, 28.4. HRMS (ESI) calcd for $\mathrm{C}_{16} \mathrm{H}_{17} \mathrm{~N}_{2} \mathrm{OS}(\mathrm{M}+$ $\mathrm{H})^{+} \mathrm{m} / z$ 285.1061, found $\mathrm{m} / z 285.1063$.

3-(2-Methylbut-3-yn-2-yl)imidazolidine-2,4-dione $\left(2 m^{\prime}\right)$. Compound $2 \mathrm{~m}^{\prime}$ was obtained from $1 \mathrm{~m}$ in $16 \mathrm{~h}$ at $50{ }^{\circ} \mathrm{C}$, according to the general procedure. The crude was purified by flash column chromatography using hexane/ethyl acetate $(1: 1)$ as eluent to give $2 \mathrm{~m}^{\prime}$ (48 $\mathrm{mg}, 72 \%$ yield) as a white solid; $\mathrm{mp}$ (hexane) $81.1-82.6{ }^{\circ} \mathrm{C}$. ${ }^{1} \mathrm{H}$ NMR $\left(400 \mathrm{MHz}, \mathrm{CDCl}_{3}\right) \delta 6.33(\mathrm{bs}, 1 \mathrm{H}), 3.88(\mathrm{~d}, J=1.2 \mathrm{~Hz}$, $2 \mathrm{H}), 2.47(\mathrm{~s}, 1 \mathrm{H}), 1.89(\mathrm{~s}, 6 \mathrm{H}) .{ }^{13} \mathrm{C}\{1 \mathrm{H}\} \mathrm{NMR}\left(101 \mathrm{MHz}, \mathrm{CDCl}_{3}\right) \delta$ 170.9 , 158.1, 85.5, 71.2, 52.3, 46.2, 29.0. HRMS (ESI) calcd for $\mathrm{C}_{8} \mathrm{H}_{11} \mathrm{~N}_{2} \mathrm{O}_{2}(\mathrm{M}+\mathrm{H})^{+} \mathrm{m} / z$ 167.0820, found $\mathrm{m} / z$ 167.0817.

(Z)-N-(4,4-Dimethyl-5-methylenethiazolidin-2-ylidene)-4-fluoroaniline $\left(\mathbf{2} n^{\prime}\right)$. Compound $2 n^{\prime}$ was synthesized as reported above, in the absence of catalyst. ${ }^{25}$ Expected acyclic thiourea 1i was not detected, and (Z)-N-(4,4-dimethyl-5-methylenethiazolidin-2-ylidene)-4-fluoroaniline $\left(\mathbf{2} \mathbf{n}^{\prime}\right)$ was instead isolated. Product $\mathbf{2} \mathbf{n}^{\prime}$ was recovered without purification in $98 \%$ yield $(1 \mathrm{mmol}, 231 \mathrm{mg})$ as a yellow solid; mp (hexane) 223.3-224.6 ${ }^{\circ} \mathrm{C} .{ }^{1} \mathrm{H}$ NMR (400 MHz, DMSO- $d_{6}$, recorded at $\left.80{ }^{\circ} \mathrm{C}\right) \delta 8.73(\mathrm{bs}, 1 \mathrm{H}), 7.54-7.32(\mathrm{~m}, 2 \mathrm{H})$, $7.16-6.88(\mathrm{~m}, 2 \mathrm{H}), 5.21(\mathrm{~d}, J=1.4 \mathrm{~Hz}, 1 \mathrm{H}), 5.14(\mathrm{~d}, J=1.4 \mathrm{~Hz}$, $1 \mathrm{H}), 1.42(\mathrm{~s}, 6 \mathrm{H}) .{ }^{13} \mathrm{C}\{1 \mathrm{H}\}$ NMR $\left(101 \mathrm{MHz}\right.$, DMSO-d $d_{6}$, recorded at $\left.80{ }^{\circ} \mathrm{C}\right) \delta 157.0\left(\mathrm{~d}, J_{\mathrm{C}, \mathrm{F}}=238.3 \mathrm{~Hz}\right), 154.7,149.5,139.8,120.0\left(\mathrm{~d}, J_{\mathrm{C}, \mathrm{F}}\right.$ $=7.1 \mathrm{~Hz}), 114.6\left(\mathrm{~d}, J_{\mathrm{C}, \mathrm{F}}=22.2 \mathrm{~Hz}\right), 101.6,74.4,29.5 .{ }^{19} \mathrm{~F}\{1 \mathrm{H}\} \mathrm{NMR}$ $\left(376 \mathrm{MHz}, \mathrm{DMSO}-d_{6}\right) \delta-121.82$. HRMS (ESI) calcd for $\mathrm{C}_{12} \mathrm{H}_{13} \mathrm{FN}_{2} \mathrm{NaS}(\mathrm{M}+\mathrm{Na})^{+} \mathrm{m} / z 259.0681$, found $\mathrm{m} / z 259.0683$.

1,4-Dibenzyl-3,5-diphenyl-1H-imidazol-2(3H)-one (4a). Compound $4 \mathrm{a}$ was obtained from $3 \mathrm{a}$ in $1 \mathrm{~min}$, according to the general procedure. The crude was purified by flash column chromatography using hexane/ethyl acetate (6:4) as eluent to give $\mathbf{4 a}$ (139 $\mathrm{mg}, 83 \%$ yield) as a pale yellow oil. The spectroscopic data of $4 \mathbf{a}$ were consistent with literature values. ${ }^{8 \mathrm{a}}{ }^{1} \mathrm{H}$ NMR $\left(400 \mathrm{MHz}, \mathrm{CDCl}_{3}\right) \delta$ 7.43-7.36 (m, 3H), 7.35-7.23 (m, 8H), 7.20-7.15 (m, 2H), 7.14$7.08(\mathrm{~m}, 5 \mathrm{H}), 6.81-6.72(\mathrm{~m}, 2 \mathrm{H}), 4.92(\mathrm{~s}, 2 \mathrm{H}), 3.71(\mathrm{~s}, 2 \mathrm{H})$. ${ }^{13} \mathrm{C}\{1 \mathrm{H}\} \mathrm{NMR}\left(75 \mathrm{MHz}, \mathrm{CDCl}_{3}\right) \delta 153.6,138.2,137.9,135.2,130.4$, $129.1,129.0,128.7,128.6,128.5,128.2,128.1,128.0,127.8,127.8$, 127.3, 126.3, 122.3, 119.1, 45.5, 29.8. HRMS (ESI) calcd for $\mathrm{C}_{29} \mathrm{H}_{25} \mathrm{~N}_{2} \mathrm{O}(\mathrm{M}+\mathrm{H})^{+} \mathrm{m} / z$ 417.1967, found $\mathrm{m} / z$ 417.1970.

1-Benzyl-4-nonyl-3,5-diphenyl-1H-imidazol-2(3H)-one (4b). Compound $\mathbf{4 b}$ was obtained from $3 \mathbf{b}$ in $1 \mathrm{~min}$, according to the general procedure. After $16 \mathrm{~h}$, the yield of $\mathbf{4 b}$ did not improve and most of the starting material was recovered $(70 \%)$. The crude was purified by flash column chromatography using hexane/ethyl acetate (7:3) as eluent to give $4 \mathbf{b}\left(33 \mathrm{mg}, 18 \%\right.$ yield) as a pale orange oil. ${ }^{1} \mathrm{H}$ NMR $\left(400 \mathrm{MHz}, \mathrm{CDCl}_{3}\right) \delta 7.50-7.45(\mathrm{~m}, 2 \mathrm{H}), 7.42-7.40(\mathrm{~m}, 1 \mathrm{H})$, 7.40-7.38 (m, 1H), 7.38-7.34 (m, 4H), 7.23-7.15 (m, 5H), 7.06$7.02(\mathrm{~m}, 2 \mathrm{H}), 4.81(\mathrm{~s}, 2 \mathrm{H}), 2.35-2.31(\mathrm{~m}, 2 \mathrm{H}), 1.27-0.90(\mathrm{~m}, 14 \mathrm{H})$, $0.85(\mathrm{t}, J=7.2 \mathrm{~Hz}, 3 \mathrm{H}) .{ }^{13} \mathrm{C}\{1 \mathrm{H}\} \mathrm{NMR}\left(101 \mathrm{MHz}, \mathrm{CDCl}_{3}\right) \delta 153.5$, $138.0,135.7,130.6,129.5,129.3,128.6,128.4,128.4,127.9,127.8$ (2C), 127.3, 121.1, 120.8, 45.4, 31.9, 29.3, 29.2, 28.9, 28.7, 28.4, 23.5, 22.7, 14.2. HRMS (ESI) calcd for $\mathrm{C}_{31} \mathrm{H}_{37} \mathrm{~N}_{2} \mathrm{O}(\mathrm{M}+\mathrm{H})^{+} \mathrm{m} / z$ 453.2906, found $m / z$ 453.2902.

4-Benzyl-1-butyl-3,5-diphenyl-1H-imidazol-2(3H)-one (4c). Compound $4 \mathrm{c}$ was obtained from $3 \mathrm{c}$ in $1 \mathrm{~min}$, according to the general procedure. The crude was purified by flash column chromatography using hexane/ethyl acetate $(7: 3)$ as eluent to give 4c (108 $\mathrm{mg}, 71 \%$ yield) as a white solid; $\mathrm{mp}$ (hexane) 112.6-113.7 ${ }^{\circ} \mathrm{C} .{ }^{1} \mathrm{H}$ NMR $\left(400 \mathrm{MHz}, \mathrm{CDCl}_{3}\right) \delta 7.48-7.36(\mathrm{~m}, 5 \mathrm{H}), 7.31-7.23$ $(\mathrm{m}, 3 \mathrm{H}), 7.14-7.05(\mathrm{~m}, 5 \mathrm{H}), 6.73(\mathrm{dd}, J=6.6,2.8 \mathrm{~Hz}, 2 \mathrm{H}), 3.75-$ $3.70(\mathrm{~m}, 2 \mathrm{H}), 3.68(\mathrm{~s}, 2 \mathrm{H}), 1.56-1.47(\mathrm{~m}, 2 \mathrm{H}), 1.28-1.17(\mathrm{~m}, 2 \mathrm{H})$, $0.81(\mathrm{t}, J=7.4 \mathrm{~Hz}, 3 \mathrm{H}) .{ }^{13} \mathrm{C}\{1 \mathrm{H}\} \mathrm{NMR}\left(101 \mathrm{MHz}, \mathrm{CDCl}_{3}\right) \delta 153.2$, 138.3 , 135.2, 130.0, 129.4, 128.9, 128.8, 128.5, 128.2, 128.0, 127.9, 
127.7, 126.2, 122.2, 118.7, 41.5, 31.3, 29.7, 19.8, 13.6. HRMS (ESI) calcd for $\mathrm{C}_{26} \mathrm{H}_{27} \mathrm{~N}_{2} \mathrm{O}(\mathrm{M}+\mathrm{H})^{+} m / z$ 383.2123, found $m / z 383.2126$. 4-Benzyl-3,5-diphenyl-1-(1-phenylethyl)-1H-imidazol-2(3H)-one (4d). Compound $4 \mathrm{~d}$ was obtained from $3 \mathrm{~d}$ in $1 \mathrm{~min}$, according to the general procedure. The crude was purified by flash column chromatography using hexane/ethyl acetate $(6: 4)$ as eluent to give 4d (160 mg, 93\% yield) as pale orange oil. ${ }^{1} \mathrm{H}$ NMR $(400 \mathrm{MHz}$, $\left.\mathrm{CDCl}_{3}\right) \delta 7.40-7.35(\mathrm{~m}, 3 \mathrm{H}), 7.35-7.26(\mathrm{~m}, 8 \mathrm{H}), 7.24-7.20(\mathrm{~m}$, $2 \mathrm{H}), 7.15(\mathrm{dd}, J=7.9,1.4 \mathrm{~Hz}, 2 \mathrm{H}), 7.13-7.08(\mathrm{~m}, 3 \mathrm{H}), 6.74(\mathrm{dd}, J=$ 6.4, $2.6 \mathrm{~Hz}, 2 \mathrm{H}), 5.29(\mathrm{q}, J=7.2 \mathrm{~Hz}, 1 \mathrm{H}), 3.72-3.56(\mathrm{~m}, 2 \mathrm{H}), 1.86$ $(\mathrm{d}, J=7.2 \mathrm{~Hz}, 3 \mathrm{H}) .{ }^{13} \mathrm{C}\{1 \mathrm{H}\} \mathrm{NMR}\left(101 \mathrm{MHz}, \mathrm{CDCl}_{3}\right) \delta 153.1$, $141.7,138.2,135.0,131.0,129.3,128.8,128.6,128.5,128.3,128.1$, 128.0, 127.9, 127.6, 127.1, 127.0, 126.2, 122.3, 119.0, 53.0, 29.8, 18.6. HRMS (ESI) calcd for $\mathrm{C}_{30} \mathrm{H}_{27} \mathrm{~N}_{2} \mathrm{O}(\mathrm{M}+\mathrm{H})^{+} \mathrm{m} / z$ 431.2123, found $m / z 431.2120$.

4-Benzyl-1-(4-methylbenzyl)-3,5-diphenyl-1H-imidazol-2(3H)one (4e). Compound $4 \mathrm{e}$ was obtained from $3 \mathrm{e}$ in $1 \mathrm{~min}$, according to the general procedure. The crude was purified by flash column chromatography using hexane/ethyl acetate $(6: 4)$ as eluent to give 4 e (156 $\mathrm{mg}, 91 \%$ yield) as an orange solid; $\mathrm{mp}$ (hexane) 146.3-148.1 ${ }^{\circ} \mathrm{C}$. ${ }^{1} \mathrm{H}$ NMR $\left(400 \mathrm{MHz}, \mathrm{CDCl}_{3}\right) \delta 7.41-7.36(\mathrm{~m}, 3 \mathrm{H}), 7.35-7.27$ (m, 5H), 7.18-7.14 (m, 2H), 7.12-7.04 (m, 5H), $7.01(\mathrm{~d}, J=8.1 \mathrm{~Hz}$, $2 \mathrm{H}), 6.75(\mathrm{dd}, J=6.3,2.7 \mathrm{~Hz}, 2 \mathrm{H}), 4.88(\mathrm{~s}, 2 \mathrm{H}), 3.70(\mathrm{~s}, 2 \mathrm{H}), 2.32$ $(\mathrm{s}, 3 \mathrm{H}) .{ }^{13} \mathrm{C}\{1 \mathrm{H}\}$ NMR $\left(101 \mathrm{MHz}, \mathrm{CDCl}_{3}\right) \delta 153.5,138.2,136.8$, $135.1,134.9,130.4,129.1$ (2C), 128.9, 128.7, 128.5, 128.2, 128.0, $127.9,127.8,127.7,126.2,122.3,119.0,45.2,29.7,21.1$. HRMS (ESI) calcd for $\mathrm{C}_{30} \mathrm{H}_{27} \mathrm{~N}_{2} \mathrm{O}(\mathrm{M}+\mathrm{H})^{+} m / z$ 431.2123, found $m / z 431.2121$.

4-Benzyl-1-(4-fluorobenzyl)-3,5-diphenyl-1H-imidazol-2(3H)-one (4f). Compound $4 \mathrm{f}$ was obtained from $3 \mathrm{f}$ in $1 \mathrm{~min}$, according to the general procedure. The crude was purified by flash column chromatography using hexane/ethyl acetate $(6: 4)$ as eluent to give 4f (161 mg, 93\% yield) as a dark yellow oil. ${ }^{1} \mathrm{H}$ NMR $(400 \mathrm{MHz}$, $\left.\mathrm{CDCl}_{3}\right) \delta 7.42-7.38(\mathrm{~m}, 3 \mathrm{H}), 7.35-7.24(\mathrm{~m}, 5 \mathrm{H}), 7.15(\mathrm{dd}, J=7.7$, $1.4 \mathrm{~Hz}, 2 \mathrm{H}), 7.12-7.03(\mathrm{~m}, 5 \mathrm{H}), 6.93(\mathrm{t}, J=8.7 \mathrm{~Hz}, 2 \mathrm{H}), 6.72(\mathrm{dd}, J$ $=6.2,2.7 \mathrm{~Hz}, 2 \mathrm{H}), 4.86(\mathrm{~s}, 2 \mathrm{H}), 3.69(\mathrm{~s}, 2 \mathrm{H}) .{ }^{13} \mathrm{C}\{1 \mathrm{H}\} \mathrm{NMR}(101$ $\left.\mathrm{MHz}, \mathrm{CDCl}_{3}\right) \delta 162.05\left(\mathrm{~d}, J_{\mathrm{C}, \mathrm{F}}=245.5 \mathrm{~Hz}\right), 153.4,138.0,135.0$, $133.6\left(\mathrm{~d}, J_{\mathrm{C}, \mathrm{F}}=3.2 \mathrm{~Hz}\right), 130.3,129.6\left(\mathrm{~d}, J_{\mathrm{C}, \mathrm{F}}=8.1 \mathrm{~Hz}\right), 128.9,128.7$, $128.6,128.2,128.0,127.9,127.8,126.2,122.0,119.2,115.20\left(\mathrm{~d}, J_{\mathrm{C}, \mathrm{F}}=\right.$ $21.4 \mathrm{~Hz}), 44.7,29.7 .{ }^{19} \mathrm{~F}\{1 \mathrm{H}\}$ NMR $\left(376 \mathrm{MHz}, \mathrm{CDCl}_{3}\right) \delta-115.09$. HRMS (ESI) calcd for $\mathrm{C}_{29} \mathrm{H}_{24} \mathrm{FN}_{2} \mathrm{O}(\mathrm{M}+\mathrm{H})^{+} \mathrm{m} / z$ 435.1873, found $m / z 435.1875$.

1,4-Bibenzyl-3-phenyl-5-(p-tolyl)-1H-imidazol-2(3H)-one (4g). Compound $4 \mathrm{~g}$ was obtained from $3 \mathrm{~g}$ in $1 \mathrm{~min}$, according to the general procedure. The crude was purified by flash column chromatography using hexane/ethyl acetate (6:4) as eluent to give $4 \mathrm{~g}$ (144 mg, 84\% yield) as a dark yellow solid; mp (hexane) $125.8-$ $127.3^{\circ} \mathrm{C}$. The spectroscopic data of $4 \mathrm{~g}$ were consistent with literature values. ${ }^{35}{ }^{1} \mathrm{H}$ NMR $\left(400 \mathrm{MHz}, \mathrm{CDCl}_{3}\right) \delta 7.37-7.26(\mathrm{~m}, 7 \mathrm{H}), 7.23-$ $7.15(\mathrm{~m}, 5 \mathrm{H}), 7.15-7.10(\mathrm{~m}, 4 \mathrm{H}), 7.07(\mathrm{~s}, 1 \mathrm{H}), 6.79(\mathrm{dd}, J=6.7,2.3$ $\mathrm{Hz}, 2 \mathrm{H}), 4.93(\mathrm{~s}, 2 \mathrm{H}), 3.74(\mathrm{~s}, 2 \mathrm{H}), 2.34(\mathrm{~s}, 3 \mathrm{H}) .{ }^{13} \mathrm{C}\{1 \mathrm{H}\} \mathrm{NMR}$ $\left(101 \mathrm{MHz}, \mathrm{CDCl}_{3}\right) \delta 153.5,138.2,138.0,135.2,131.1,129.3,128.9$, $128.5,128.4,128.1,128.0,127.9,127.8,127.7,127.4,127.2,126.2$, 122.5, 118.8, 45.5, 29.7, 21.3. HRMS (ESI) calcd for $\mathrm{C}_{30} \mathrm{H}_{27} \mathrm{~N}_{2} \mathrm{O}(\mathrm{M}$ $+\mathrm{H})^{+} \mathrm{m} / z$ 431.2123, found $\mathrm{m} / z 431.2120$.

1,4-Dibenzyl-5-(4-methoxyphenyl)-3-phenyl-1H-imidazol-2(3H)one (4h). Compound $4 \mathrm{~h}$ was obtained from $3 \mathrm{~h}$ in $1 \mathrm{~min}$, according to the general procedure. The crude was purified by flash column chromatography using hexane/ethyl acetate (6:4) as eluent to give $\mathbf{4 h}$ (137 mg, 77\% yield) as a white solid; $\mathrm{mp}$ (hexane) $166.6-168.3^{\circ} \mathrm{C}$. ${ }^{1} \mathrm{H}$ NMR $\left(400 \mathrm{MHz}, \mathrm{CDCl}_{3}\right) \delta 7.37-7.24(\mathrm{~m}, 6 \mathrm{H}), 7.24-7.15(\mathrm{~m}$, $6 \mathrm{H}), 7.15-7.09(\mathrm{~m}, 3 \mathrm{H}), 6.95-6.89(\mathrm{~m}, 2 \mathrm{H}), 6.79(\mathrm{dd}, J=7.1,2.2$ $\mathrm{Hz}, 2 \mathrm{H}), 4.91(\mathrm{~s}, 2 \mathrm{H}), 3.82(\mathrm{~s}, 3 \mathrm{H}), 3.71(\mathrm{~s}, 2 \mathrm{H}) .{ }^{13} \mathrm{C}\{1 \mathrm{H}\} \mathrm{NMR}$ $\left(101 \mathrm{MHz}, \mathrm{CDCl}_{3}\right) \delta 159.7,153.3,138.2,137.9,135.1,131.7,128.8$, $128.3,128.1,127.9,127.8,127.6$ (2C), 127.1, 126.1, 121.9, 121.0, 118.6, 114.0, 55.2, 45.3, 29.6. HRMS (ESI) calcd for $\mathrm{C}_{30} \mathrm{H}_{27} \mathrm{~N}_{2} \mathrm{O}_{2}$ (M $+\mathrm{H})^{+} \mathrm{m} / z$ 447.2072, found $\mathrm{m} / z$ 447.2074.

1,4-Dibenzyl-5-(3-chlorophenyl)-3-phenyl-1H-imidazol-2(3H)one (4i). Compound $4 \mathbf{i}$ was obtained from $3 \mathbf{i}$ in $1 \mathrm{~min}$, according to the general procedure. The crude was purified by flash column chromatography using hexane/ethyl acetate $(6: 4)$ as eluent to give $\mathbf{4 i}$
(155 mg, 86\% yield) as an orange solid; mp (hexane) 130.5-132.7 ${ }^{\circ} \mathrm{C} .{ }^{1} \mathrm{H}$ NMR $\left(400 \mathrm{MHz}, \mathrm{CDCl}_{3}\right) \delta 7.38-7.25(\mathrm{~m}, 8 \mathrm{H}), 7.22(\mathrm{t}, J=$ $1.4 \mathrm{~Hz}, 1 \mathrm{H}), 7.18-7.09(\mathrm{~m}, 8 \mathrm{H}), 6.73(\mathrm{dd}, J=6.5,2.4 \mathrm{~Hz}, 2 \mathrm{H}), 4.89$ $(\mathrm{s}, 2 \mathrm{H}), 3.69(\mathrm{~s}, 2 \mathrm{H}) .{ }^{13} \mathrm{C}\{1 \mathrm{H}\} \mathrm{NMR}\left(101 \mathrm{MHz}, \mathrm{CDCl}_{3}\right) \delta 153.4$, $137.6,137.5,134.8,134.4,130.7,130.2,129.9,128.9,128.6,128.4$, $128.3,128.2,127.9,127.8,127.7,127.6,127.3,126.3,120.9,119.7$, 45.5, 29.6. HRMS (ESI) calcd for $\mathrm{C}_{29} \mathrm{H}_{24} \mathrm{ClN}_{2} \mathrm{O}(\mathrm{M}+\mathrm{H})^{+} \mathrm{m} / z$ 451.1577, found $m / z$ 451.1581.

1,4-Dibenzyl-3-phenyl-5-(3-vinylphenyl)-1H-imidazol-2(3H)-one (4j). Compound $4 \mathbf{j}$ was obtained from $3 \mathbf{j}$ in $1 \mathrm{~min}$, according to the general procedure. The crude was purified by flash column chromatography using hexane/ethyl acetate (6:4) as eluent to give 4j (133 mg, 75\% yield) as a pale yellow solid; mp (hexane) $143.8-$ $145.2{ }^{\circ} \mathrm{C} .{ }^{1} \mathrm{H}$ NMR $\left(400 \mathrm{MHz}, \mathrm{CDCl}_{3}\right) \delta 7.43(\mathrm{~d}, J=7.8 \mathrm{~Hz}, 1 \mathrm{H})$, $7.38-7.32(\mathrm{~m}, 4 \mathrm{H}), 7.32-7.26(\mathrm{~m}, 4 \mathrm{H}), 7.23-7.16(\mathrm{~m}, 5 \mathrm{H}), 7.15-$ $7.11(\mathrm{~m}, 3 \mathrm{H}), 6.80(\mathrm{dd}, J=6.8,2.2 \mathrm{~Hz}, 2 \mathrm{H}), 6.66(\mathrm{dd}, J=17.6,10.9$ $\mathrm{Hz}, 1 \mathrm{H}), 5.62(\mathrm{~d}, J=17.6 \mathrm{~Hz}, 1 \mathrm{H}), 5.27(\mathrm{~d}, J=10.9 \mathrm{~Hz}, 1 \mathrm{H}), 4.93(\mathrm{~s}$, $2 \mathrm{H}), 3.74(\mathrm{~s}, 2 \mathrm{H}) .{ }^{13} \mathrm{C}\{1 \mathrm{H}\} \mathrm{NMR}\left(101 \mathrm{MHz}, \mathrm{CDCl}_{3}\right) \delta 153.6,138.2$, 138.02, 137.97, 136.1, 135.1, 129.6, 129.3, 129.0, 128.9, 128.5, 128.3, $128.14,128.08,127.97,127.85,127.77,127.3,126.5,126.3,122.2$, 119.1, 114.8, 45.7, 29.8. HRMS (ESI) calcd for $\mathrm{C}_{31} \mathrm{H}_{27} \mathrm{~N}_{2} \mathrm{O}(\mathrm{M}+\mathrm{H})^{+}$ $\mathrm{m} / z$ 443.2123, found $\mathrm{m} / z$ 443.2120.

1,4-Dibenzyl-5-(2-bromophenyl)-3-phenyl-1H-imidazol-2(3H)one (4k). Compound $4 \mathbf{k}$ was obtained from $3 \mathbf{k}$ in $1 \mathrm{~min}$, according to the general procedure. The crude was purified by flash column chromatography using hexane/ethyl acetate (7:3) as eluent to give $\mathbf{4 k}$ (134 mg, 68\% yield) as a dark yellow oil. ${ }^{1} \mathrm{H}$ NMR $(400 \mathrm{MHz}$, $\left.\mathrm{CDCl}_{3}\right) \delta 7.68(\mathrm{dd}, J=7.3,1.8 \mathrm{~Hz}, 1 \mathrm{H}), 7.39-7.31(\mathrm{~m}, 3 \mathrm{H}), 7.30-$ $7.19(\mathrm{~m}, 7 \mathrm{H}), 7.14(\mathrm{dd}, J=6.7,2.5 \mathrm{~Hz}, 1 \mathrm{H}), 7.10-7.06(\mathrm{~m}, 3 \mathrm{H})$, $7.05-7.01(\mathrm{~m}, 2 \mathrm{H}), 6.79(\mathrm{dd}, J=6.4,2.7 \mathrm{~Hz}, 2 \mathrm{H}), 5.12(\mathrm{~d}, J=15.2$ $\mathrm{Hz}, 1 \mathrm{H}), 4.60(\mathrm{~d}, J=15.2 \mathrm{~Hz}, 1 \mathrm{H}), 3.71-3.52(\mathrm{~m}, 2 \mathrm{H}) .{ }^{13} \mathrm{C}\{1 \mathrm{H}\}$ NMR $\left(101 \mathrm{MHz}, \mathrm{CDCl}_{3}\right) \delta 153.1,137.5,137.4,135.0,133.8,132.9$, $130.7,130.1,128.9,128.2,128.1,128.1,128.0,127.9,127.8,127.3$, 127.2, 126.4, 126.1, 120.4, 119.7, 45.6, 30.0. HRMS (ESI) calcd for $\mathrm{C}_{29} \mathrm{H}_{24} \mathrm{BrN}_{2} \mathrm{O}(\mathrm{M}+\mathrm{H})^{+} \mathrm{m} / z$ 495.1072, found $\mathrm{m} / z$ 495.1071.

1,4-Dibenzyl-5-phenyl-3-propyl-1H-imidazol-2(3H)-one (4I). Compound 41 was obtained from 31 in $1 \mathrm{~min}$, according to the general procedure. After $16 \mathrm{~h}$, the yield remain unchanged and $42 \%$ of the starting material was recovered. The crude was purified by flash column chromatography using hexane/ethyl acetate (6:4) as eluent to give 41 (50 mg, 33\% yield) as a yellowish oil. ${ }^{1} \mathrm{H}$ NMR $(400 \mathrm{MHz}$, $\left.\mathrm{CDCl}_{3}\right) \delta 7.34-7.28(\mathrm{~m}, 5 \mathrm{H}), 7.27-7.21(\mathrm{~m}, 4 \mathrm{H}), 7.20-7.16(\mathrm{~m}$, $2 \mathrm{H}), 7.15-7.11(\mathrm{~m}, 2 \mathrm{H}), 7.05(\mathrm{dd}, J=7.8,1.6 \mathrm{~Hz}, 2 \mathrm{H}), 4.87(\mathrm{~s}, 2 \mathrm{H})$, $3.81(\mathrm{~s}, 2 \mathrm{H}), 3.48-3.43(\mathrm{~m}, 2 \mathrm{H}), 1.60-1.49(\mathrm{~m}, 2 \mathrm{H}), 0.85(\mathrm{t}, J=7.4$ $\mathrm{Hz}, 3 \mathrm{H}) .{ }^{13} \mathrm{C}\{1 \mathrm{H}\}$ NMR $\left(101 \mathrm{MHz}, \mathrm{CDCl}_{3}\right) \delta 154.0,138.4,138.2$, $130.2,129.2$, 128.8, 128.6, 128.4, 128.3, 127.8, 127.4, 127.2, 126.8, 121.7, 118.1, 45.4, 43.45, 29.6, 22.8, 11.3. HRMS (ESI) calcd for $\mathrm{C}_{26} \mathrm{H}_{27} \mathrm{~N}_{2} \mathrm{O}(\mathrm{M}+\mathrm{H})^{+} \mathrm{m} / z$ 383.2123, found $\mathrm{m} / z 383.2127$.

1,3,4-tribenzyl-5-phenyl-1H-imidazol-2(3H)-one (4m). Compound $4 \mathrm{~m}$ was obtained from $3 \mathrm{~m}$ in $1 \mathrm{~min}$, according to the general procedure. The crude was purified by flash column chromatography using hexane/ethyl acetate (6:4) as eluent to give $4 \mathrm{~m}$ (144 mg, $84 \%$ yield) as a yellow oil. Spectroscopic data of $4 \mathbf{m}$ were consistent with literature values. ${ }^{341} \mathrm{H}$ NMR $\left(400 \mathrm{MHz}, \mathrm{CDCl}_{3}\right) \delta 7.37-7.25(\mathrm{~m}$, $12 \mathrm{H}), 7.24-7.18(\mathrm{~m}, 4 \mathrm{H}), 7.14-7.07(\mathrm{~m}, 4 \mathrm{H}), 4.96(\mathrm{~s}, 2 \mathrm{H}), 4.72(\mathrm{~s}$, $2 \mathrm{H}), 3.65(\mathrm{~s}, 2 \mathrm{H}) .{ }^{13} \mathrm{C}\{1 \mathrm{H}\} \mathrm{NMR}\left(101 \mathrm{MHz}, \mathrm{CDCl}_{3}\right) \delta 154.4,138.1$, $138.0,137.9,130.1,129.0,128.9,128.7,128.6,128.5,128.4,127.8$, $127.4,127.3,127.2,127.1,126.8,122.2,118.0,45.6,44.9,29.5$. HRMS (ESI) calcd for $\mathrm{C}_{30} \mathrm{H}_{27} \mathrm{~N}_{2} \mathrm{O}(\mathrm{M}+\mathrm{H})^{+} \mathrm{m} / z$ 431.2123, found $m / z 431.2125$.

1-Benzyl-4-(4-methylbenzyl)-3,5-diphenyl-1H-imidazol-2(3H)one (4n). Compound $4 \mathrm{n}$ was obtained from $3 \mathrm{n}$ in $1 \mathrm{~min}$, according to the general procedure. The crude was purified by flash column chromatography using hexane/ethyl acetate (6:4) as eluent to give $4 \mathbf{n}$ (139 mg, 81\% yield) as a yellow solid; mp (hexane) $128.4-129.4{ }^{\circ} \mathrm{C}$. ${ }^{1} \mathrm{H}$ NMR (400 MHz, $\left.\mathrm{CDCl}_{3}\right) \delta 7.45-7.33(\mathrm{~m}, 6 \mathrm{H}), 7.32-7.25(\mathrm{~m}$, $5 \mathrm{H}), 7.24-7.18(\mathrm{~m}, 2 \mathrm{H}), 7.18-7.11(\mathrm{~m}, 2 \mathrm{H}), 6.94(\mathrm{~d}, J=7.9 \mathrm{~Hz}$, $2 \mathrm{H}), 6.66(\mathrm{~d}, J=7.9 \mathrm{~Hz}, 2 \mathrm{H}), 4.93(\mathrm{~s}, 2 \mathrm{H}), 3.68(\mathrm{~s}, 2 \mathrm{H}), 2.28(\mathrm{~s}$, $3 \mathrm{H}) .{ }^{13} \mathrm{C}\{1 \mathrm{H}\} \mathrm{NMR}\left(101 \mathrm{MHz}, \mathrm{CDCl}_{3}\right) \delta 153.6,137.9,135.7,135.1$, $130.3,129.1,129.0,128.9,128.7,128.5,128.4,128.1,127.8$ (2C), 
127.7, 127.3, 122.1, 119.2, 45.5, 29.2, 21.0. HRMS (ESI) calcd for $\mathrm{C}_{30} \mathrm{H}_{27} \mathrm{~N}_{2} \mathrm{O}(\mathrm{M}+\mathrm{H})^{+} m / z$ 431.2123, found $m / z$ 431.2122.

1-Benzyl-4-(4-chlorobenzyl)-3,5-diphenyl-1H-imidazol-2(3H)one (4o). Compound $4 \mathrm{o}$ was obtained from $3 \mathrm{o}$ in $1 \mathrm{~min}$, according to the general procedure. The crude was purified by flash column chromatography using hexane/ethyl acetate $(7: 3)$ as eluent to give $\mathbf{4 o}$ (176 mg, 98\% yield) as a viscous light yellow oil. ${ }^{1} \mathrm{H}$ NMR $(400 \mathrm{MHz}$, $\left.\mathrm{CDCl}_{3}\right) \delta 7.42-7.30(\mathrm{~m}, 6 \mathrm{H}), 7.29-7.22(\mathrm{~m}, 5 \mathrm{H}), 7.19(\mathrm{~d}, J=7.3$ $\mathrm{Hz}, 2 \mathrm{H}), 7.14-7.03(\mathrm{~m}, 4 \mathrm{H}), 6.66(\mathrm{~d}, J=8.3 \mathrm{~Hz}, 2 \mathrm{H}), 4.91(\mathrm{~s}, 2 \mathrm{H})$, $3.67(\mathrm{~s}, 2 \mathrm{H}) .{ }^{13} \mathrm{C}\{1 \mathrm{H}\}$ NMR $\left(101 \mathrm{MHz}, \mathrm{CDCl}_{3}\right) \delta 153.5,137.8$, 136.7, 135.1, 132.0, 130.3, 129.3, 129.1, 128.9, 128.8, 128.7, 128.4 128.3, 128.0, 127.9, 127.8, 127.3, 122.5, 118.5, 45.5, 29.2. HRMS (ESI) calcd for $\mathrm{C}_{29} \mathrm{H}_{24} \mathrm{ClN}_{2} \mathrm{O}(\mathrm{M}+\mathrm{H})^{+} \mathrm{m} / z$ 451.1577, found $\mathrm{m} / z$ 451.1576.

1-Benzyl-4-(4-fluorobenzyl)-3,5-diphenyl-1H-imidazol-2(3H)-one (4p). Compound $4 p$ was obtained from $3 p$ in $1 \mathrm{~min}$, according to the general procedure. The crude was purified by flash column chromatography using hexane/ethyl acetate $(7: 3)$ as eluent to give $4 \mathbf{p}(168 \mathrm{mg}, 97 \%$ yield $)$ as a viscous oil. ${ }^{1} \mathrm{H}$ NMR $\left(400 \mathrm{MHz}, \mathrm{CDCl}_{3}\right)$ $\delta 7.43-7.23(\mathrm{~m}, 11 \mathrm{H}), 7.22-7.17(\mathrm{~m}, 2 \mathrm{H}), 7.15-7.10(\mathrm{~m}, 2 \mathrm{H})$, 6.82-6.75 (m, 2H), 6.72-6.65 (m, 2H), $4.93(\mathrm{~s}, 2 \mathrm{H}), 3.69(\mathrm{~s}, 2 \mathrm{H})$. ${ }^{13} \mathrm{C}\{1 \mathrm{H}\} \operatorname{NMR}\left(101 \mathrm{MHz}, \mathrm{CDCl}_{3}\right) \delta 161.3\left(\mathrm{~d}, J_{\mathrm{C}, \mathrm{F}}=244.6 \mathrm{~Hz}\right)$, 153.4, 137.8, 135.1, 133.7 (d, $\left.J_{\mathrm{C}, \mathrm{F}}=3.2 \mathrm{~Hz}\right), 130.3,129.3\left(\mathrm{~d}, J_{\mathrm{C}, \mathrm{F}}=7.9\right.$ $\mathrm{Hz}), 129.0,128.9,128.7,128.6,128.3,127.9,127.8,127.7,127.2$, 122.3, 118.9, $114.9\left(\mathrm{~d}, J_{\mathrm{C}, \mathrm{F}}=21.3 \mathrm{~Hz}\right), 45.4,28.9 .{ }^{19} \mathrm{~F}\{1 \mathrm{H}\} \mathrm{NMR}$ $\left(376 \mathrm{MHz}, \mathrm{CDCl}_{3}\right) \delta-116.60$. HRMS (ESI) calcd for $\mathrm{C}_{29} \mathrm{H}_{24} \mathrm{FN}_{2} \mathrm{O}$ $(\mathrm{M}+\mathrm{H})^{+} \mathrm{m} / z$ 435.1873, found $\mathrm{m} / z$ 435.1874.

\section{ASSOCIATED CONTENT}

\section{S Supporting Information}

The Supporting Information is available free of charge on the ACS Publications website at DOI: 10.1021/acs.joc.9b00064.

Copy of NMR spectra, computational details and single crystal X-ray diffraction data for $\mathbf{2 a}, \mathbf{2 i}(\mathrm{Z}), \mathbf{2 n} \mathbf{n}^{\prime}, \mathbf{4 c}$, and 4j (PDF)

\section{AUTHOR INFORMATION}

\section{Corresponding Authors}

*E-mail: stirling.andras@ttk.mta.hu.

*E-mail: nicola.dellaca@unipr.it.

\section{ORCID $\odot$}

Alessia Bacchi: 0000-0001-5675-9372

Raffaella Mancuso: 0000-0001-7514-6096

Bartolo Gabriele: 0000-0003-4582-1489

Raimondo Maggi: 0000-0003-3811-7186

Giovanni Maestri: 0000-0002-0244-8605

András Stirling: 0000-0002-1696-7932

Nicola Della Ca': 0000-0002-7853-7954

\section{Notes}

The authors declare no competing financial interest.

\section{ACKNOWLEDGMENTS}

We wish to thank the University of Parma and MIUR (Ministry of Education, University and Research, FFABR 2017) for financial support and CIM (Interdepartmental Measurements Centre) for the NMR facilities. Chiesi Farmaceutici $\mathrm{SpA}$ is acknowledged for the support with the D8 Venture X-ray equipment. We are thankful for the support from the COST (European Cooperation in Science and Technology) Action (CA15106) on $\mathrm{C}-\mathrm{H}$ Activation in Organic Synthesis. A.S. acknowledges support from Grant K116034 of the NRDI Office, Hungary, and the computational resources of KIFÜ, Hungary.

\section{REFERENCES}

(1) (a) Lee, K.-C.; Venkateswararao, E.; Sharma, V. K.; Jung, S.-H. Investigation of Amino Acid Conjugates of (S)-1-[1-(4-Aminobenzoyl)-2,3-Dihydro-1H-Indol-6-Sulfonyl]-4-Phenyl-Imidazolidin-2One (DW2282) as Water Soluble Anticancer Prodrugs. Eur. J. Med. Chem. 2014, 80, 439-446. (b) Chang, C.-S.; Lin, Y.-T.; Shih, S.-R.; Lee, C.-C.; Lee, Y.-C.; Tai, C.-L.; Tseng, S.-N.; Chern, J.-H. Design, Synthesis, and Antipicornavirus Activity of 1-[5-(4-Arylphenoxy)alkyl]-3-pyridin-4-ylimidazolidin-2-one Derivatives. J. Med. Chem. 2005, 48, 3522-3535. (c) Dage, R. C.; Roebel, L. E.; Gibson, J. P.; Okerholm, R. A.; Rolf, C. N. Enoximone. Cardiovasc. Drug Rev. 1986, 4, 63-79. (d) Reichard, G. A.; Stengone, C.; Paliwal, S.; Mergelsberg, I.; Majmundar, S.; Wang, C.; Tiberi, R.; McPhail, A. T.; Piwinski, J. J.; Shih, N.-Y. Asymmetric Synthesis of 4,4-Disubstituted-2-Imidazolidinones: Potent NK ${ }_{1}$ Antagonists. Org. Lett. 2003, 5, 4249-4251. (e) Pettit, G. R.; McNulty, J.; Herald, D. L.; Doubek, D. L.; Chapuis, J.-C.; Schmidt, J. M.; Tackett, L. P.; Boyd, M. R. Antineoplastic Agents. 362. Isolation and X-Ray Crystal Structure of Dibromophakellstatin from the Indian Ocean Sponge Phakellia Mauritiana. J. Nat. Prod. 1997, 60, 180-183. (f) De Clercq, P. J. Biotin: A Timeless Challenge for Total Synthesis. Chem. Rev. 1997, 97, 1755-1792.

(2) (a) Espinoza-Hicks, C.; Montoya, P.; Bautista, R.; JiménezVázquez, H. A.; Rodríguez-Valdez, L. M.; Camacho-Dávila, A. A.; Cossío, F. P.; Delgado, F.; Tamariz, J. Synthesis of Exo -Imidazolidin2-One Dienes, Their Isomerization, and Selectivity in Diels-Alder Cycloadditions. J. Org. Chem. 2018, 83, 5347-5364. (b) Koswatta, P.; Sivappa, R.; Dias, H.; Lovely, C. Total Synthesis of the LeucettaDerived Alkaloid Calcaridine A. Synthesis 2009, 2009, 2970-2982. (c) Vasanthakumar, G. R.; Bhor, V. M.; Surolia, A. Hydrolysis of Cyclic Ureas under Microwave Irradiation: Synthesis and Characterization of 7,8 Diaminopelargonic Acid. Synth. Commun. 2007, 37, 2633-2639. (d) Barrios Sosa, A. C.; Yakushijin, K.; Horne, D. A. Synthesis of Slagenins A, B, and C. Org. Lett. 2000, 2, 3443-3444.

(3) (a) Lu, C.; Hu, L.; Yang, G.; Chen, Z. 2-Imidazolidinones as Chiral Auxiliaries in Asymmetric Synthesis. Curr. Org. Chem. 2012, 16, 2802-2817. (b) Guillena, G.; Nájera, C. (4R,5S)-1,5-Dimethyl-4Phenylimidazolidin-2-One as a Chiral Auxiliary for the Diastereoselective Alkylation of a New Iminic Glycine Derivative: Practical Asymmetric Synthesis of $\alpha$-Amino Acids. Tetrahedron: Asymmetry 1998, 9, 1125-1129. (c) Matsunaga, H.; Ishizuka, T.; Kunieda, T. Synthetic Utility of Five-Membered Heterocycles-Chiral Functionalization and Applications. Tetrahedron 2005, 61, 8073-8094.

(4) (a) Casnati, A.; Motti, E.; Mancuso, R.; Gabriele, B.; Della Ca', $\mathrm{N}$. Recent Advances in the Catalytic Synthesis of Imidazolidin-2-Ones and Benzimidazolidin-2-Ones. Catalysts 2019, 9, 28. (b) Sartori, G.; Maggi, R. In Science of Synthesis (Houben-Weyl Methods of Molecular Transformations), Vol. 18; Ley, S. V., Knight, J. G., Eds.; Thieme: Stuttgart, 2005; pp 665-758. For selected examples on the use of $\mathrm{CO}$ as carbonylating agent, see: (c) Qian, F.; McCusker, J. E.; Zhang, Y.; Main, A. D.; Chlebowski, M.; Kokka, M.; McElwee-White, L. Catalytic Oxidative Carbonylation of Primary and Secondary Diamines to Cyclic Ureas. Optimization and Substituent Studies. J. Org. Chem. 2002, 67, 4086-4092. (d) Casiello, M.; Iannone, F.; Cotugno, P.; Monopoli, A.; Cioffi, N.; Ciminale, F.; Trzeciak, A. M.; Nacci, A. Copper(II)-Catalysed Oxidative Carbonylation of Aminols and Amines in Water: A Direct Access to Oxazolidinones, Ureas and Carbamates. J. Mol. Catal. A: Chem. 2015, 407, 8-14. (e) McCusker, J. E.; Grasso, C. A.; Main, A. D.; McElwee-White, L. Catalytic Oxidative Carbonylation of Primary and Secondary $\alpha, \omega$-Diamines to Cyclic Ureas. Org. Lett. 1999, 1, 961-964. (f) Gabriele, B.; Salerno, G.; Mancuso, R.; Costa, M. Efficient Synthesis of Ureas by Direct Palladium-Catalyzed Oxidative Carbonylation of Amines. J. Org. Chem. 2004, 69, 4741-4750. For a selected example on the use of dialkylcarbonates as carbonylating agents, see: (g) Ballini, R.; Fiorini, D.; Maggi, R.; Righi, P.; Sartori, G.; Sartorio, R. TBD-Catalysed Solventless Synthesis of Symmetrically N,N'-Substituted Ureas from Primary Amines and Diethyl Carbonate. Green Chem. 2003, 5, 396398. For a recent review and representative examples on the use of $\mathrm{CO}_{2}$ as carbonylating agent, see: (h) Tamura, M.; Honda, M.; 
Nakagawa, Y.; Tomishige, K. Direct Conversion of $\mathrm{CO}_{2}$ with Diols, Aminoalcohols and Diamines to Cyclic Carbonates, Cyclic Carbamates and Cyclic Ureas Using Heterogeneous Catalysts: Direct Conversion of $\mathrm{CO}_{2}$ with Diols. J. Chem. Technol. Biotechnol. 2014, 89, 19-33. (i) Yu, B.; Zhang, H.; Zhao, Y.; Chen, S.; Xu, J.; Hao, L.; Liu, Z. DBU-Based Ionic-Liquid-Catalyzed Carbonylation of $o$-Phenylenediamines with $\mathrm{CO}_{2}$ to 2-Benzimidazolones under Solvent-Free Conditions. ACS Catal. 2013, 3, 2076-2082. (j) Kimura, T.; Kamata, K.; Mizuno, N. A Bifunctional Tungstate Catalyst for Chemical Fixation of $\mathrm{CO}_{2}$ at Atmospheric Pressure. Angew. Chem., Int. Ed. 2012, 51, 6700-6703. (k) Tamura, M.; Noro, K.; Honda, M.; Nakagawa, Y.; Tomishige, K. Highly Efficient Synthesis of Cyclic Ureas from $\mathrm{CO}_{2}$ and Diamines by a Pure $\mathrm{CeO}_{2}$ Catalyst Using a 2-Propanol Solvent. Green Chem. 2013, 15, 1567-1577. (1) Paz, J.; Pérez-Balado, C.; Iglesias, B.; Muñoz, L. Carbon Dioxide as a Carbonylating Agent in the Synthesis of 2-Oxazolidinones, 2-Oxazinones, and Cyclic Ureas: Scope and Limitations. J. Org. Chem. 2010, 75, 3037-3046. (m) Wu, C.; Cheng, H.; Liu, R.; Wang, Q.; Hao, Y.; Yu, Y.; Zhao, F. Synthesis of Urea Derivatives from Amines and $\mathrm{CO}_{2}$ in the Absence of Catalyst and Solvent. Green Chem. 2010, 12, 1811-1816. (n) Bhanage, B. M.; Fujita, S.; Ikushima, Y.; Arai, M. Synthesis of Cyclic Ureas and Urethanes from Alkylene Diamines and Amino Alcohols with Pressurized Carbon Dioxide in the Absence of Catalysts. Green Chem. 2003, 5, 340-342.

(5) (a) Zhu, Y.; Cornwall, R. G.; Du, H.; Zhao, B.; Shi, Y. Catalytic Diamination of Olefins via N-N Bond Activation. Acc. Chem. Res. 2014, 47, 3665-3678. (b) Zhu, Y.; Shi, Y. Cu-Catalyzed Sequential Diamination and Dehydrogenation of Terminal Olefins: A Facile Approach to Imidazolinones. Chem. - Eur. J. 2014, 20, 13901-13904.

(6) (a) Tabarki, M. A.; Besbes, R. Ring Expansion of Aziridine-2Carboxylates. An Efficient Entry to Imidazolidin-2-Ones and Oxazolidin-2-Imines. Tetrahedron Lett. 2015, 56, 1837-1839. (b) Kennedy, M. D.; Bailey, S. J.; Wales, S. M.; Keller, P. A. Enantiopure Trans -4,5-Disubstituted 2-Imidazolidinones via Copper(I)-Catalyzed Ring Opening of 1,1'-DiBoc-2,2'-Biaziridine with Grignard Reagents. J. Org. Chem. 2015, 80, 5992-5998. (c) Xing, M.-L.; Lu, X.-W.; Miao, C.-B.; Li, J.-X.; Sun, X.-Q.; Yang, H.-T. Lewis Base-Catalyzed Reaction of Aziridinofullerene with Ureas for the Preparation of Fulleroimidazolidinones. J. Org. Chem. 2014, 79, 11774-11779.

(7) (a) Patel, M.; Saunthwal, R. K.; Verma, A. K. Base-Mediated Hydroamination of Alkynes. Acc. Chem. Res. 2017, 50 (2), 240-254. (b) Yamamoto, C.; Hirano, K.; Miura, M. Cesium HydroxideMediated Regio- and Stereoselective Hydroamidation of Internal Aryl Alkynes with Primary Amides. Chem. Lett. 2017, 46, 1048-1050. (c) Tzalis, D.; Knochel, P. Cesium Hydroxide: A Superior Base for the Catalytic Alkynylation of Aldehydes and Ketones and Catalytic Alkenylation of Nitriles. Angew. Chem., Int. Ed. 1999, 38, 1463-1465. (d) Tzalis, D.; Koradin, C.; Knochel, P. Cesium Hydroxide Catalyzed Addition of Alcohols and Amine Derivatives to Alkynes and Styrene. Tetrahedron Lett. 1999, 40, 6193-6195.

(8) For transition-metal-catalyzed intramolecular hydroamidation to five-membered cyclic ureas, see: (a) Huang, L.; Arndt, M.; Gooßen, K.; Heydt, H.; Gooßen, L. J. Late Transition Metal-Catalyzed Hydroamination and Hydroamidation. Chem. Rev. 2015, 115, 25962697. (b) Rajesh, M.; Puri, S.; Kant, R.; Sridhar Reddy, M. AgCatalyzed Intramolecular Sequential Vicinal Diamination of Alkynes with Isocyanates: Synthesis of Fused Indole-Cyclic Urea Derivatives. J. Org. Chem. 2017, 82, 5169-5177. (c) Pereshivko, O. P.; Peshkov, V. A.; Jacobs, J.; Meervelt, L. V.; Van der Eycken, E. V. Cationic Goldand Silver-Catalyzed Cycloisomerizations of Propargylic Ureas: A Selective Entry to Oxazolidin-2-Imines and Imidazolidin-2-Ones. Adv. Synth. Catal. 2013, 355, 781-789. (d) Peshkov, V. A.; Pereshivko, O. P.; Sharma, S.; Meganathan, T.; Parmar, V. S.; Ermolat'ev, D. S.; Van der Eycken, E. V. Tetrasubstituted 2-Imidazolones via $\mathrm{Ag}(\mathrm{I})$ Catalyzed Cycloisomerization of Propargylic Ureas. J. Org. Chem. 2011, 76, 5867-5872. (e) Lei, A.; Lu, X. Palladium(II)-Catalyzed Tandem Intramolecular Aminopalladation of Alkynes and Conjugate Addition. Synthesis of Oxazolidinones, Imidazolidinones, and
Lactams. Org. Lett. 2000, 2, 2699-2702. (f) Bacchi, A.; Chiusoli, G. P.; Costa, M.; Sani, C.; Gabriele, B.; Salerno, G. Nitrogen Heterocycles by Palladium-Catalysed Oxidative Cyclization-Alkoxycarbonylation of Acetylenic Ureas. J. Organomet. Chem. 1998, 562, 35-43. (g) Fritz, J. A.; Nakhla, J. S.; Wolfe, J. P. A New Synthesis of Imidazolidin-2-Ones via Pd-Catalyzed Carboamination of $N$-Allylureas. Org. Lett. 2006, 8, 2531-2534. (h) Huang, S.; Shao, Y.; Liu, R.; Zhou, X. Facile Access to Oxazolidin-2-Imine, Thiazolidin-2-Imine and Imidazolidin-2-Imine Derivatives Bearing an Exocyclic Haloalkyliene via Direct Halocyclization between Propargylamines, Heterocumulenes and $I_{2}$ (NBS). Tetrahedron 2015, 71, 4219-4226. (i) LaVenia, A.; Medran, N. S.; Krchňák, V.; Testero, S. A. Synthesis of a Small Library of Imidazolidin-2-Ones Using Gold Catalysis on Solid Phase. ACS Comb. Sci. 2016, 18, 482-489.

(9) For metal-free intramolecular hydroamidation to five-membered cyclic ureas, see: (a) Ranjan, A.; Yerande, R.; Wakchaure, P. B.; Yerande, S. G.; Dethe, D. H. Base-Mediated Hydroamination of Propargylamine: A Regioselective Intramolecular 5-exo-dig Cycloisomerization en Route to Imidazole-2-thione. Org. Lett. 2014, 16, 5788-5791. (b) Proulx, C.; Lubell, W. D. N -Amino-Imidazolin-2One Peptide Mimic Synthesis and Conformational Analysis. Org. Lett. 2012, 14, 4552-4555. (c) Huguenot, F.; Delalande, C.; Vidal, M. Metal-Free 5-Exo-Dig Cyclization of Propargyl Urea Using TBAF. Tetrahedron Lett. 2014, 55, 4632-4635. (d) Liu, J.; Zhang, Y.; Li, G.; Roschangar, F.; Farina, V.; Senanayake, C. H.; Lu, B. Z. A Novel OnePot, Two-Step Synthesis of Polycyclic Indoles via Tandem Intramolecular Hydroamidation/Palladium-Catalyzed Annulation. Adv. Synth. Catal. 2010, 352, 2667-2671. (e) Chiu, S. K.; Dube, M.; Keifer, L.; Szilagyi, S.; Timberlake, J. W. Attempted Synthesis of a Keto Diazene: Reactions of Propargylic Amines, Sulfamides, and Ureas. J. Org. Chem. 1978, 43, 61-65. (f) Easton, N. R.; Cassady, D. R.; Dillard, R. D. Reactions of Acetylenic Amines. VIII. Cyclization of Acetylenic Ureas. J. Org. Chem. 1964, 29, 1851-1855. (g) Stoffel, P. J.; Speziale, A. J. The Preparation of 2-Imidazolones: A Novel Ring Closure of Propynyl Ureas with Phosphorus Pentachloride. J. Am. Chem. Soc. 1962, 84, 501-502.

(10) (a) Khrakovsky, D. A.; Tao, C.; Johnson, M. W.; Thornbury, R. T.; Shevick, S. L.; Toste, F. D. Enantioselective, Stereodivergent Hydroazidation and Hydroamination of Allenes Catalyzed by Acyclic Diaminocarbene (ADC) Gold(I) Complexes. Angew. Chem., Int. Ed. 2016, 55, 6079-6083. (b) Kanno, O.; Kuriyama, W.; Wang, Z. J.; Toste, F. D. Regio- and Enantioselective Hydroamination of Dienes by Gold(I)/Menthol Cooperative Catalysis. Angew. Chem., Int. Ed. 2011, 50, 9919-9922. (c) Li, H.; Widenhoefer, R. A. Gold(I)Catalyzed Intramolecular Dihydroamination of Allenes with $N^{\prime}$ Disubstituted Ureas To Form Bicyclic Imidazolidin-2-ones. Org. Lett. 2009, 11, 2671-2674.

(11) Marchegiani, M.; Nodari, M.; Tansini, F.; Massera, C.; Mancuso, R.; Gabriele, B.; Costa, M.; Della Ca', N. Urea Derivatives from Carbon Dioxide and Amines by Guanidine Catalysis: Easy Access to Imidazolidin-2-ones under Solvent-Free Conditions. J. $\mathrm{CO}_{2}$ Utilization 2017, 21, 553-561.

(12) Urea 1a was prepared from 2-methylbut-3-yn-2-amine and phenyl isocyanate (see Experimental Section for details).

(13) (a) Taylor, J. E.; Bull, S. D.; Williams, J. M. J. Amidines, Isothioureas, and Guanidines as Nucleophilic Catalysts. Chem. Soc. Rev. 2012, 41, 2109-2121. (b) Coles, M. P. Bicyclic-Guanidines, -Guanidinates and -Guanidinium Salts: Wide Ranging Applications from a Simple Family of Molecules. Chem. Commun. 2009, 36593676.

(14) Kozell, V.; Rahmani, F.; Piermatti, O.; Lanari, D.; Vaccaro, L. A Stereoselective Organic Base-Catalyzed Protocol for Hydroamination of Alkynes under Solvent-Free Conditions. Molecular Catalysis 2018, $455,188-191$.

(15) CCDC 1876216 (2a), CCDC 1876217 (2i), CCDC 1876218 $\left(2 n^{\prime}\right)$, CCDC 1876219 (4c), and CCDC 1876220 (4j) contain the supplementary crystallographic data for this paper. These data can be obtained free of charge from the Cambridge Crystallographic Data Centre via www.ccdc.cam.ac.uk/data request/cif. 
(16) Frisch, M. J.; et al. Gaussian 09, revision A.02; Gaussian, Inc.: Wallingford, CT, 2009.

(17) Chai, J.-D.; Head-Gordon, M. Long-Range Corrected Hybrid Density Functionals with Damped Atom-Atom Dispersion Corrections. Phys. Chem. Chem. Phys. 2008, 10, 6615-6620.

(18) Predicted BTMG basicity was higher with respect to the others. For a similar overstabilization for BTMG, see: Turnell-Ritson, R. C.; Sapsford, J. S.; Cooper, R. T.; Lee, S. S.; Földes, T.; Hunt, P. A.; Pápai, I.; Ashley, A. E. Base-Induced Reversible $\mathrm{H}_{2}$ Addition to a Single Sn(ii) Centre. Chem. Sci. 2018, 9, 8716-8722.

(19) Ryde, U. A fundamental view of enthalpy-entropy compensation. MedChemComm 2014, 5, 1324-1336. Zhou, H.-X.; Gilson, M. K. Theory of Free Energy and Entropy in Noncovalent Binding. Chem. Rev. 2009, 109, 4092-4107.

(20) Ryu, H.; Park, J.; Kim, H. K.; Park, J. Y.; Kim, S.-T.; Baik, M.-H. Pitfalls in Computational Modeling of Chemical Reactions and How to Avoid Them. Organometallics 2018, 37, 3228-3239.

(21) Note that for clarity we indicated the level shifts only for the first two stages of the reactions although the next level is also shifted by the variation of the base. The initial and final states are not affected because the base is not protonated in these states (i.e., a spectator).

(22) Modern Allene Chemistry; Krause, N., Hashmi, A. S. K., Eds.; Wiley-VCH Verlag GmbH: Weinheim, Germany, 2004.

(23) For recent DFT studies carried out on catalytic sequences involving an allene group, see: (a) Cera, G.; Lanzi, M.; Balestri, D.; Della Ca', N.; Maggi, R.; Bigi, F.; Malacria, M.; Maestri, G. Synthesis of Carbolines via Palladium/Carboxylic Acid Joint Catalysis. Org. Lett. 2018, 20, 3220-3224. (b) Ocello, R.; De Nisi, A.; Jia, M.; Yang, Q.Q.; Monari, M.; Giacinto, P.; Bottoni, A.; Miscione, G. P.; Bandini, M. Gold(I)-Catalyzed Dearomative [2 + 2]-Cycloaddition of Indoles with Activated Allenes: A Combined Experimental-Computational Study. Chem. - Eur. J. 2015, 21, 18445-18453.

(24) The DFT calculations also addressed the suppressed reactivity of $3 q$ (see SI for the details).

(25) Liu, J.; Shen, M.; Zhang, Y.; Li, G.; Khodabocus, A.; Rodriguez, S.; Qu, B.; Farina, V.; Senanayake, C. H.; Lu, B. Z. A New Entry to Polycyclic Indole Skeletons via Palladium-Catalyzed Intramolecular Heteroannulation. Org. Lett. 2006, 8, 3573-3575.

(26) Sonogashira, K.; Tohda, Y.; Hagihara, N. A Convenient Synthesis of Acetylenes: Catalytic Substitutions of Acetylenic Hydrogen with Bromoalkenes, Iodoarenes and Bromopyridines. Tetrahedron Lett. 1975, 16, 4467-4470.

(27) Brak, K.; Doyle, P. S.; McKerrow, J. H.; Ellman, J. A. Identification of a New Class of Nonpeptidic Inhibitors of Cruzain. J. Am. Chem. Soc. 2008, 130, 6404-6410.

(28) Yoo, W.; Li, C. Copper Catalyzed Four Component Coupling between Aldehydes, Amines, Alkynes, and Carbon Dioxide. Adv. Synth. Catal. 2008, 350, 1503-1506.

(29) Chang, Z.; Jing, X.; He, C.; Liu, X.; Duan, C. Silver Clusters as Robust Nodes and $\pi$-Activation Sites for the Construction of Heterogeneous Catalysts for the Cycloaddition of Propargylamines. ACS Catal. 2018, 8, 1384-1391.

(30) Mishra, P. K.; Verma, S.; Kumar, M.; Verma, A. K. BaseMediated Direct Transformation of $N$-Propargylamines into 2,3,5Trisubstituted $1 \mathrm{H}$-Pyrroles. Org. Lett. 2018, 20, 7182-7185.

(31) Wang, H.; Ying, J.; Lai, M.; Qi, X.; Peng, J.-B.; Wu, X.-F. BasePromoted Carbonylative Cyclization of Propargylic Amines with Selenium under CO Gas-Free Conditions. Adv. Synth. Catal. 2018, 360, 1693-1703.

(32) Shi, L.; Tu, Y.-Q.; Wang, M.; Zhang, F.-M.; Fan, C.-A. Microwave-Promoted Three-Component Coupling of Aldehyde, Alkyne, and Amine via $\mathrm{C}-\mathrm{H}$ Activation Catalyzed by Copper in Water. Org. Lett. 2004, 6, 1001-1003.

(33) Bariwal, J. B.; Ermolat'ev, D. S.; VanderEycken, E. V. Efficient Microwave-Assisted Synthesis of Secondary Alkylpropargylamines by Using $\mathrm{A}^{3}$-Coupling with Primary Aliphatic Amines. Chem. - Eur. J. 2010, 16, 3281-3284.

(34) Ranjan, A.; Deore, A. S.; Yerande, S. G.; Dethe, D. H. ThiolYne Coupling of Propargylamine under Solvent-Free Conditions by
Bond Anion Relay Chemistry: An Efficient Synthesis of Thiazolidin-2Ylideneamine. Eur. J. Org. Chem. 2017, 2017, 4130-4139.

(35) Pereshivko, O. P.; Peshkov, V. A.; Peshkov, A. A.; Jacobs, J.; Van Meervelt, L.; Van der Eycken, E. V. Unexpected Regio- and Chemoselectivity of Cationic Gold-Catalyzed Cycloisomerizations of Propargylureas: Access to Tetrasubstituted 3,4-Dihydropyrimidin2(1H)-Ones. Org. Biomol. Chem. 2014, 12, 1741. 